\title{
Chain decompositions of 4-connected graphs
}

\author{
Sean Curran* $\quad$ Orlando Lee ${ }^{\dagger} \quad$ Xingxing $\mathrm{Yu}^{\ddagger}$
}

April 11, 2003

School of Mathematics

Georgia Institute of Technology

Atlanta, Georgia 30332

\begin{abstract}
In this paper we give a decomposition of a 4-connected graph $G$ into nonseparating chains, which is similar to an ear decomposition of a 2-connected graph. We also give an $O\left(|V(G)|^{2}|E(G)|\right)$ algorithm that constructs such a decomposition. In applications, the asymptotic performance can often be improved to $O\left(|V(G)|^{3}\right)$. This decomposition will be used to find four independent spanning trees in a 4connected graph.
\end{abstract}

${ }^{*}$ Partially supported by NSF VIGRE grant

${ }^{\dagger}$ Supported by CNPq (Proc: 200611/00-3) - Brazil.

${ }^{\ddagger}$ Partially supported by NSF grant DMS 9970527 and NSA grant MDA904-03-1-0052 


\section{Introduction}

In [2], Cheryian and Maheshwari gave an $O\left(|V(G)|^{2}\right)$ algorithm for finding a "nonseparating ear decomposition" of a 3-connected graph $G$, and they used this decomposition to construct three independent spanning trees in a 3-connected graph.

In this paper we give a 4-connected version of the non-separating ear decomposition of Cheriyan and Maheshwari, and an $O\left(|V(G)|^{2}|E(G)|\right)$ algorithm for finding such a decomposition. This will be used in a forthcoming paper to find four independent spanning trees in an arbitrary 4-connected graph $G$, where the asymptotic performance can be improved to $O\left(|V(G)|^{3}\right)$.

We use the definitions and notation given in [1]. Recall the definitions of chain (Definition (1.3) in [1]), planar chain (Definition (1.4) in [1]), cyclic chain (Definition (4.2) in [1]), and planar cyclic chain (Definition (4.3) in [1]).

The chains in our decomposition can be classified into four types, as described below. The first three types are planar chains as defined in (1.1). The fourth type is not a planar chain (but almost planar as we will see), and it is defined in (1.2). See Figure 1 for illustrations of (1.1) and (1.2).

(1.1) Definition. Let $G$ be a graph, let $F$ be a subgraph of $G$, and let $r \in V(F)$. Let $H$ be a planar $x-y$ chain in $G$ such that $V(H)-\{x, y\} \subseteq V(G)-V(F)$. We say that

(a) $H$ is an up F-chain if $\{x, y\} \subseteq V(F)$ and $N_{G}(H-\{x, y\}) \subseteq(V(G)-V(F-r)) \cup\{x, y\}$,

(b) $H$ is a down $F$-chain if $\{x, y\} \subseteq V(G)-V(F-r)$ and $N_{G}(H-\{x, y\}) \subseteq V(F-r) \cup$ $\{x, y\}$, and

(c) $H$ is an elementary $F$-chain if $\{x, y\} \subseteq V(F)$ and $H$ is an $x$-y path of length two.

In any of the three cases above we say that $H$ is a planar $x-y F$-chain in $G$ (or simply, a planar F-chain). For an $x$-y chain $H$ we let $I(H):=V(H)-\{x, y\}$ and for a cyclic chain $H$ we let $I(H):=V(H)$.

For a graph $G$, a subgraph $H$ of $G$, and $S \subseteq V(G) \cup E(G)$, we let $H+S$ denote the graph with vertex set $V(H) \cup(S \cap V(G))$ and edge set $E(H) \cup(S \cap E(G))$.

(1.2) Definition. Let $G$ be a graph, let $F$ be a subgraph of $G$, and let $r \in V(F)$. Suppose that $\left\{v_{1}, v_{2}, v_{3}\right\} \subseteq V(G)-V(F)$ induces a triangle $T$ in $G$, and for each $1 \leq i \leq 3$, $v_{i}$ has exactly one neighbor $x_{i}$ in $V(F-r)$ and exactly one neighbor $y_{i}$ in $V(G)-(V(F) \cup$ $V(T)$ ) (thus, each $v_{i}$ has degree four in $G$ ). Moreover, assume that $x_{1}, x_{2}, x_{3}$ are distinct and $y_{1}, y_{2}, y_{3}$ are distinct. Then we say that $H:=T+\left\{x_{1}, x_{2}, x_{3}, v_{1} x_{1}, v_{2} x_{2}, v_{3} x_{3}\right\}$ is a triangle $F$-chain in $G$. We let $I(H):=\left\{v_{1}, v_{2}, v_{3}\right\}$. 


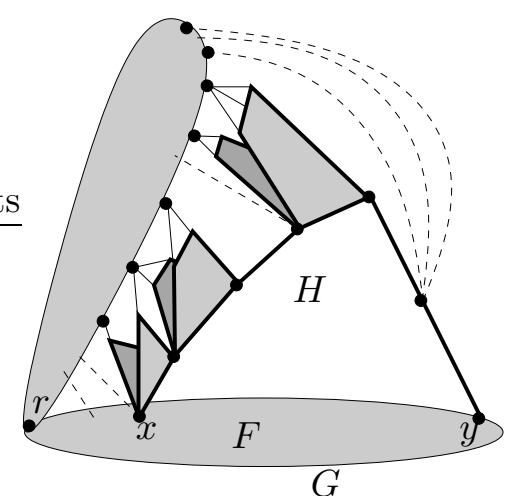

(a)

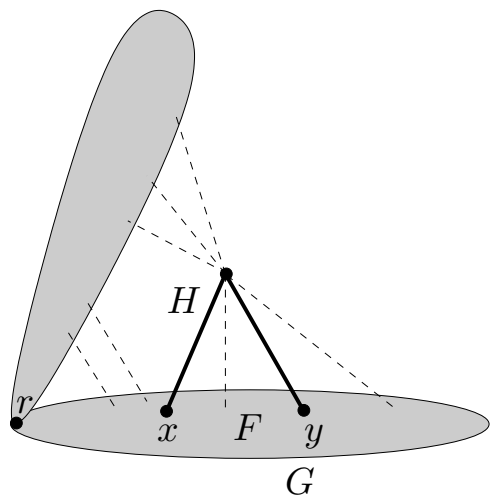

(c)

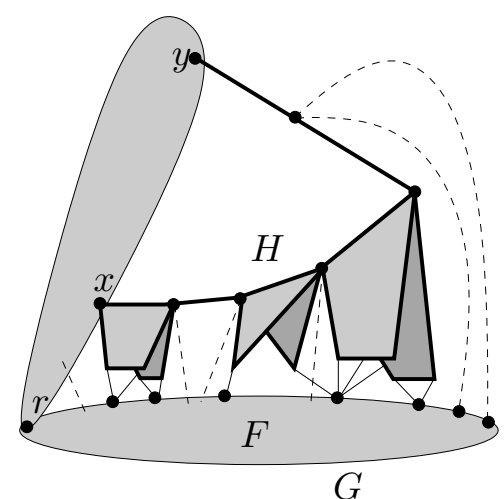

(b)

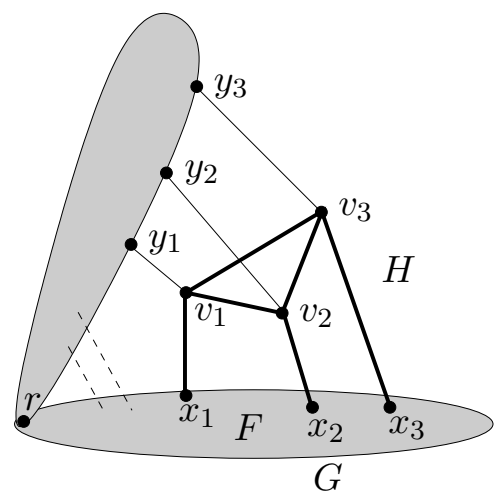

(d)

Figure 1: (a) An up $F$-chain, (b) a down $F$-chain, (c) an elementary $F$-chain, and (d) a triangle $F$-chain. The dashed edges need not exist.

The definitions above depend on the choice of $r$ and $F$, but in spite of this, whenever we use these concepts in this paper, it should be clear which pair $r, F$ we refer to.

(1.3) Definition. Let $G$ be a graph, let $F$ be a subgraph of $G$, and let $r \in V(F)$. By a good $F$-chain in $G$, we mean an up $F$-chain, or a down $F$-chain, or an elementary $F$-chain, or a triangle $F$-chain.

We are now ready to describe a chain decomposition, which is similar to an ear decomposition.

(1.4) Definition. Let $G$ be a graph, let $r \in V(G)$, and let $H_{1}, \ldots, H_{t}$ be chains in $G$, where $t \geq 2$. We say that $\left(H_{1}, \ldots, H_{t}\right)$ is a non-separating chain decomposition of $G$ 
rooted at $r$ if the following conditions hold:

(i) $H_{1}$ is a planar cyclic chain in $G$ rooted at $r$,

(ii) for each $i=2, \ldots, t-1, H_{i}$ is a good $G\left[\bigcup_{j=1}^{i-1} I\left(H_{j}\right)\right]$-chain in $G$,

(iii) $H_{t}=G-\left(\bigcup_{j=1}^{t-1} I\left(H_{j}\right)-\{r\}\right)$ is a planar cyclic chain in $G$ rooted at $r$, and

(iv) for each $i=1, \ldots, t-1$, both $G\left[\bigcup_{j=1}^{i} I\left(H_{j}\right)\right]$ and $G-\left(\bigcup_{j=1}^{i} I\left(H_{j}\right)-\{r\}\right)$ are 2-connected.

The chains $H_{2}, \ldots, H_{t-1}$ are called internal chains of the non-separating chain decomposition. If $r a$ is a piece of $H_{1}$, then we say that $H_{1}, \ldots, H_{t}$ is a non-separating chain decomposition of $G$ starting at ra.

The main result of this paper is the following.

(1.5) Theorem. Let $G$ be a 4-connected graph, let $r \in V(G)$, and let $r a \in E(G)$. Then $G$ has a non-separating chain decomposition rooted at $r$ starting at ra, and such a decomposition can be found in $O\left(|V(G)|^{2}|E(G)|\right)$ time.

The existence of the first chain $H_{1}$ of the chain decomposition is guaranteed by the next result which corresponds to Theorem (4.4) in [1].

(1.6) Theorem. Let $G$ be a 4-connected graph and let $r a \in E(G)$. Then there exists a planar cyclic chain $H$ in $G$ rooted at $r$ such that $r a$ is a piece of $H$ and $G-(V(H)-\{r\})$ is 2-connected. Moreover, such a chain can be found in in $O(|V(G)||E(G)|)$ time.

In order to construct the internal chains of the chain decomposition in (1.5), we need the following result which is Theorem (1.6) in [1].

(1.7) Theorem. Let $G$ be a graph, let $\{a, b\} \subseteq V(G)$, and let $P$ be a non-separating induced $a-b$ path in $G$. Let $B_{P}$ be a nontrivial block of $G-V(P)$, and let $X_{P}:=$ $N_{G}\left(G-V\left(B_{P}\right)\right)$. Suppose $G-\left(V\left(B_{P}\right)-X_{P}\right)$ is $\left(4, X_{P} \cup\{a, b\}\right)$-connected. Then there exists a planar $a-b$ chain $H$ in $G$ such that $G-V(H)$ is 2-connected and $B_{P} \subseteq G-V(H)$. Moreover, such a chain can be found in $O(|V(G)||E(G)|)$ time.

The rest of this paper is organized as follows. In Section 2 we recall some lemmas proved in [1] and provide some new auxiliary lemmas concerning non-separating induced paths. In Section 3 we prove a technical result, which will be used to find the internal chains of a non-separating chain decomposition. Finally, in Section 4 we complete the proof of Theorem (1.5). 


\section{Non-separating paths}

In this section we state and prove some results concerning non-separating induced paths which will be used later. First, we state two lemmas without proof, which are Lemmas (2.3) and (2.4) in [1], respectively.

(2.1) Lemma. Let $G$ be a connected graph, $S \subseteq V(G),\left\{a, a^{\prime}\right\} \subseteq S$, and let $P$ be an $a-a^{\prime}$ path in $G$. Suppose

(i) $G$ is $(3, S)$-connected, and

(ii) $S-\left\{a, a^{\prime}\right\}$ is contained in a component $U$ of $G-V(P)$.

Then there exists a non-separating induced $a-a^{\prime}$ path $P^{\prime}$ in $G$ such that $V\left(P^{\prime}\right) \cap V(U)=\emptyset$. Moreover, such a path can be found in $O(|V(G)|+|E(G)|)$ time.

(2.2) Lemma. Let $G$ be a graph and $S:=\left\{a, a^{\prime}, b, b^{\prime}\right\} \subseteq V(G)$. Suppose that $G$ is $(4, S)$-connected. Then exactly one of the following holds:

(1) there exists a non-separating induced $a-a^{\prime}$ path $P^{\prime}$ in $G$ such that $V\left(P^{\prime}\right) \cap\left\{b, b^{\prime}\right\}=\emptyset$;

(2) $\left(G, a, b, a^{\prime}, b^{\prime}\right)$ is planar.

Moreover, one can in $O(|V(G)|+|E(G)|)$ time find a path as in (i) or certify that (ii) holds.

Note our use of "prime" notation in the statements of the lemmas. The reader should not infer that the paths labelled $P^{\prime}$ are derived from an assumed path $P$. We reserve $P$ to denote a particular path specified in Section 3, and we therefore label paths $P^{\prime}$ in the statements of our lemmas. We hope this will sidestep any source of confusion when these lemmas are applied.

The next lemma is a variation of (2.1) (and (2.2) as well) in which we prove the existence of a specific non-separating induced path. However, here it is not possible to specify the ends of the desired path. Moreover, in the hypotheses of (2.3) there are some technical conditions which arise when we try to produce an internal chain. Note that conditions (iii), (iv) and (v) are automatically satisfied if $G$ is $\left(4, S \cup\left\{b, b^{\prime}\right\}\right.$ )-connected. Actually, this is the case in all applications of (2.3) with the exception of the proof of (3.15), where the more complicated conditions are required.

(2.3) Lemma. Let $G$ be a graph, let $S \subset V(G)$, and let $\left\{b, b^{\prime}\right\} \subseteq V(G)-S$. Suppose

(i) $G-S$ is 2-connected,

(ii) every element of $S$ has a neighbor in $V(G)-\left(S \cup\left\{b, b^{\prime}\right\}\right)$, 
(iii) $G$ is $\left(3, S \cup\left\{b, b^{\prime}\right\}\right)$-connected,

(iv) if $|S|=2$, then $G$ is $\left(4, S \cup\left\{b, b^{\prime}\right\}\right)$-connected, and

(v) if $|S| \geq 3$, then there exists some component of $G-\left(S \cup\left\{b, b^{\prime}\right\}\right)$ which has at least two neighbors in $S$.

Then exactly one of the following holds:

(1) there exist $a, a^{\prime} \in S$ and an induced $a-a^{\prime}$ path $P^{\prime}$ in $G$ such that $V\left(P^{\prime}\right) \cap\left\{b, b^{\prime}\right\}=\emptyset$, $V\left(P^{\prime}\right) \cap S=\left\{a, a^{\prime}\right\}$, and $G-\left(V\left(P^{\prime}\right) \cup S\right)$ is connected;

(2) $|S|=2$, and the elements of $S$ can be labeled as $a, a^{\prime}$ such that $\left(G, a, b, a, b^{\prime}\right)$ is planar.

Moreover, one can in $O(|V(G)|+|E(G)|)$ time find a path as in (1) or certify that (2) holds.

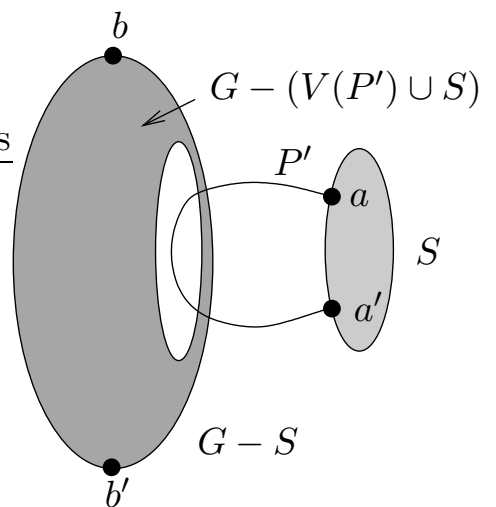

(1)

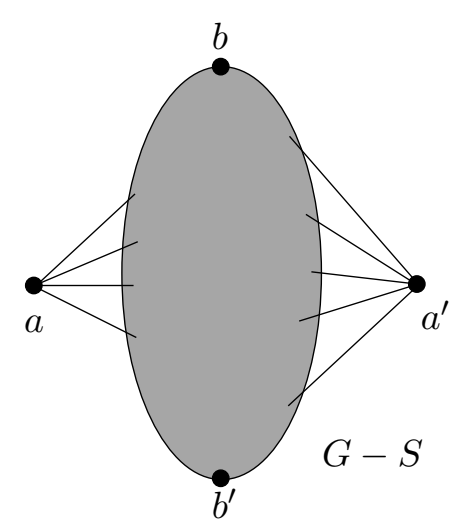

(2) $S=\left\{a, a^{\prime}\right\}$

Figure 2: Outcomes in (2.3).

Proof. First, suppose that $|S|=2$. Let $a, a^{\prime}$ denote the vertices in $S$. By (iv) $G$ is $\left(4,\left\{a, a, b, b^{\prime}\right\}\right)$-connected. Thus, by Lemma (2.2) exactly one of the following holds:

(a) there exists a non-separating induced $a$-a path $P^{\prime}$ such that $V\left(P^{\prime}\right) \cap\left\{b, b^{\prime}\right\}=\emptyset$, or

(b) $\left(G, a, b, a^{\prime}, b^{\prime}\right)$ is planar. 
Moreover, one can in $O(|V(G)|+|E(G)|)$ time find a path as in (a) or certify that (b) holds. If (a) holds, then $P^{\prime}$ is the required path in (1). If (b) holds, then (2) holds.

Thus, we may assume that $|S| \geq 3$. First, we prove the following

Claim. There exist $a, a^{*} \in S$ and an $a-a^{*}$ path $Q$ in $G-\left(S-\left\{a, a^{*}\right\}\right)$ such that $b$ and $b^{\prime}$ are contained in a component of $G-V(Q)$. Moreover, such a path can be found in $O(|V(G)|+|E(G)|)$ time.

Proof of Claim. We consider two cases.

Case 1: $G-\left(S \cup\left\{b, b^{\prime}\right\}\right)$ is not connected.

In this case, there exist edge-disjoint subgraphs $G_{1}, G_{2}$ of $G-S$ such that $G_{1} \cup G_{2}=$ $G-S, V\left(G_{1}\right) \cap V\left(G_{2}\right)=\left\{b, b^{\prime}\right\}$, and $\left|V\left(G_{1}\right)\right| \geq 3 \leq\left|V\left(G_{2}\right)\right|$. Note that such a partition can be found in $O(|V(G)|+|E(G)|)$ time.

By (v) there exists some component $K$ of $G-\left(S \cup\left\{b, b^{\prime}\right\}\right)$ which has at least two neighbors in $S$. Note that such a component can be found in $O(|V(G)|+|E(G)|)$ time. We may assume that $V(K) \subset V\left(G_{1}\right)$. Let $a, a^{*} \in N_{G}(K) \cap S$ and let $Q$ be an $a$ - $a^{*}$ path in $G\left[V(K) \cup\left\{a, a^{*}\right\}\right]$. Since $G_{2}$ is connected, $b, b^{\prime}$ are contained in a component of $G-V(Q)$. Moreover, such a path can be found in $O(|V(G)|+|E(G)|)$ time.

Case 2: $G-\left(S \cup\left\{b, b^{\prime}\right\}\right)$ is connected.

Since $G-S$ is 2-connected by (i), one can find in $O(|V(G)|+|E(G)|)$ time two internally disjoint $b-b^{\prime}$ paths $P_{1}, P_{2}$ in $G-S$. Let $a_{1}, a_{2}, a_{3}$ be distinct vertices in $S$. For $i=1,2,3$, let $v_{i} \in N_{G}\left(a_{i}\right) \subseteq V(G)-\left(S-\left\{b, b^{\prime}\right\}\right)$ (they exist by (ii)). Since $G-\left(S \cup\left\{b, b^{\prime}\right\}\right)$ is connected by assumption, for each $i=1,2,3$, there exists a path $Q_{i}$ from $v_{i}$ to some vertex $u_{i}$ in $\left(V\left(P_{1}\right) \cup V\left(P_{2}\right)\right)-\left\{b, b^{\prime}\right\}$ internally disjoint from $V\left(P_{1}\right) \cup V\left(P_{2}\right)$. Moreover, such paths can be found in $O(|V(G)|+|E(G)|)$ time. Note that at least two (not necessarily distinct) vertices in $u_{1}, u_{2}, u_{3}$ lie on the same path $P_{1}-\left\{b, b^{\prime}\right\}$ or $P_{2}-\left\{b, b^{\prime}\right\}$. By symmetry, we may assume that $u_{1}, u_{2} \in V\left(P_{1}\right)-\left\{b, b^{\prime}\right\}$. Then there exist disjoint paths in $G-\left(S-\left\{a_{1}, a_{2}\right\}\right)$ from $a_{1}$ to $a_{2}$ (the path contained in $\left.Q_{1} \cup Q_{2} \cup\left(P_{1}-\left\{b, b^{\prime}\right\}\right)\right)$ and from $b$ to $b^{\prime}$ (the path $P_{2}$ ), respectively. Thus, the result follows by taking $a=a_{1}$ and $a^{*}=a_{2}$. Moreover, it is not hard to see that such paths can be found in $O(|V(G)|+|E(G)|)$ time.

Now given $a, a^{*}$ and $Q$, we will describe how to find $a^{\prime} \in S$ and an induced $a-a^{\prime}$ path $P^{\prime}$ such that $V\left(P^{\prime}\right) \cap\left\{b, b^{\prime}\right\}=\emptyset, V\left(P^{\prime}\right) \cap S=\left\{a, a^{\prime}\right\}$, and $G-\left(V\left(P^{\prime}\right) \cup S\right)$ is connected. Let $G^{\prime}$ be the graph obtained from $G$ by identifying the vertices in $S-\{a\}$ to a single vertex $a^{\prime \prime}$ and removing the resulting multiple edges. Let $S^{\prime}:=\left\{a, a^{\prime \prime}, b, b^{\prime}\right\}$.

We claim that $G^{\prime}$ is $\left(3, S^{\prime}\right)$-connected. Suppose for a contradiction that there exists $T \subset V\left(G^{\prime}\right)$ such that $|T| \leq 2$ and $G^{\prime}-T$ has a component $K$ with $V(K) \cap S^{\prime}=\emptyset$. Clearly $a^{\prime \prime} \in T$, because $G$ is $\left(3, S \cup\left\{b, b^{\prime}\right\}\right)$-connected (by (iii)); then either $a \in T$ or $T-\left\{a^{\prime \prime}\right\}$ is a vertex cut of $G-S$, which is a contradiction since $G-S$ is 2-connected (by (i)). Thus, $G^{\prime}$ is $\left(3, S^{\prime}\right)$-connected. 
Note that the $a-a^{*}$ path $Q$ in $G$ corresponds to an $a-a^{\prime \prime}$ path $P$ in $G^{\prime}$, and $S^{\prime}-\left\{a, a^{\prime \prime}\right\}=$ $\left\{b, b^{\prime}\right\}$ is contained in a component $U$ of $G^{\prime}-V(P)$. Thus, the hypotheses of (2.1) are satisfied with $G^{\prime}, S^{\prime}, P, a, a^{\prime \prime}, U$ as $G, S, P, a, a^{\prime}, U$ respectively. Hence, there exists a nonseparating induced $a$ - $a^{\prime \prime}$ path $P^{\prime \prime}$ in $G^{\prime}$ such that $V\left(P^{\prime \prime}\right) \cap V(U)=\emptyset$. Moreover, such a path $P^{\prime \prime}$ can be found in $O\left(\left|V\left(G^{\prime}\right)\right|+\left|E\left(G^{\prime}\right)\right|\right.$ ) time (hence, in $O(|V(G)|+|E(G)|)$ time). The path $P^{\prime \prime}$ corresponds to an induced $a$-a path $P^{\prime}$ in $G$ for some $a^{\prime} \in S-\{a\}$ such that $V\left(P^{\prime}\right) \cap\left\{b, b^{\prime}\right\}=\emptyset$ and $V\left(P^{\prime}\right) \cap S=\left\{a, a^{\prime}\right\}$. Since $P^{\prime \prime}$ is non-separating in $G^{\prime}$, $G-\left(V\left(P^{\prime}\right) \cup S\right)$ is connected. Therefore, $a, a^{\prime}$ and $P^{\prime}$ satisfy (1), and they can be found in $O(|V(G)|+|E(G)|)$ time.

The following lemma is a variation of (2.3) (by letting $b=b^{\prime}$ ) and its proof is essentially the same. For the sake of completeness, we include it here.

(2.4) Lemma. Let $G$ be a graph, let $S \subset V(G)$, and let $b \in V(G)-S$. Suppose

(i) $G-S$ is 2-connected,

(ii) every element of $S$ has a neighbor in $V(G)-(S \cup\{b\})$, and

(iii) $G$ is $(3, S \cup\{b\})$-connected.

Then there exist $a, a^{\prime} \in S$ and an induced $a-a^{\prime}$ path $P^{\prime}$ in $G$ such that $V\left(P^{\prime}\right) \cap\{b\}=\emptyset$, $V\left(P^{\prime}\right) \cap S=\left\{a, a^{\prime}\right\}$, and $G-\left(V\left(P^{\prime}\right) \cup S\right)$ is connected. Moreover, such a path can be found in $O(|V(G)|+|E(G)|)$ time.

Proof. Since $G$ is (3, $S \cup\{b\}$ )-connected (by (iii)), $|S| \geq 2$, so let $a, a^{*} \in S$. Since $G-S$ is 2-connected (by (i)), $G-(S \cup\{b\})$ is connected. Since $a$ and $a^{*}$ have a neighbor in $V(G)-(S \cup\{b\})$ (by (ii)), there exists an $a-a^{*}$ path $Q$ in $G-\left(\left(S-\left\{a, a^{*}\right\}\right) \cup\{b\}\right)$. Clearly, such a path can be found in $O(|V(G)|+|E(G)|)$ time.

Let $G^{\prime}$ be the graph obtained from $G$ by identifying the vertices in $S-\{a\}$ to a single vertex $a^{\prime \prime}$ and removing the resulting multiple edges. Let $S^{\prime}:=\left\{a, a^{\prime \prime}, b\right\}$.

We claim that $G^{\prime}$ is $\left(3, S^{\prime}\right)$-connected. Suppose for a contradiction that there exists $T \subset V\left(G^{\prime}\right)$ such that $|T| \leq 2$ and $G^{\prime}-T$ has a component $K$ with $V(K) \cap S^{\prime}=\emptyset$. Clearly, $a^{\prime \prime} \in T$ because $G$ is $(3, S \cup\{b\}$ )-connected (by (iii)). But then either $a \in T$ or $T-\left\{a^{\prime \prime}\right\}$ is a vertex cut of $G-S$, which is a contradiction since $G-S$ is 2-connected (by (i)). Thus, $G^{\prime}$ is $\left(3, S^{\prime}\right)$-connected.

Note that the $a-a^{*}$ path $Q$ in $G$ corresponds to an $a-a^{\prime \prime}$ path $P$ in $G^{\prime}$, and $S^{\prime}-$ $\left\{a, a^{\prime \prime}\right\}=\{b\}$ is contained in a component $U$ of $G^{\prime}-V(P)$. Thus, by (2.1) (with $G^{\prime}, S^{\prime}, P, a, a^{\prime \prime}, U$ as $G, S, P, a, a^{\prime}, U$ respectively), there exists a non-separating induced $a-a^{\prime \prime}$ path $P^{\prime \prime}$ in $G^{\prime}$ such that $V\left(P^{\prime \prime}\right) \cap V(U)=\emptyset$. Moreover, such a path $P^{\prime \prime}$ can be found in $O\left(\left|V\left(G^{\prime}\right)\right|+\left|E\left(G^{\prime}\right)\right|\right)$ time (and hence, in $O(|V(G)|+|E(G)|)$ time). The path $P^{\prime \prime}$ corresponds to an induced $a-a^{\prime}$ path $P^{\prime}$ in $G$ for some $a^{\prime} \in S-\{a\}$ such that 
$V\left(P^{\prime}\right) \cap\{b\}=\emptyset$ and $V\left(P^{\prime}\right) \cap S=\left\{a, a^{\prime}\right\}$. Since $P^{\prime \prime}$ is non-separating in $G^{\prime}, G-\left(V\left(P^{\prime}\right) \cup S\right)$ is connected. So $a, a^{\prime}$ and $P^{\prime}$ are as required, and they can be found in $O(|V(G)|+|E(G)|)$ time.

Some results and algorithms which we use here require that we find an embedding of a planar graph $(G, a, b, c, d)$ in a closed disc such that $a, b, c, d$ occur on the boundary of the disc in that cyclic order. This can be done in linear time using an algorithm of Hopcroft and Tarjan [7] (or a more recent algorithm by Hsu and Shi [8]). For convenience, we state this result as our next lemma.

(2.5) Lemma. Let $(G, a, b, c, d)$ be a planar graph. Then one can find in $O(|V(G)|+$ $|E(G)|)$ time an embedding of $G$ in a closed disc such that $a, b, c, d$ occur on the boundary of the disc in that cyclic order.

Let $\left(G, a, b, a^{\prime}, b^{\prime}\right)$ be a planar graph. Then any $a-a^{\prime}$ path in $G-\left\{b, b^{\prime}\right\}$ separates $b$ from $b^{\prime}$. The next lemma shows that one can find efficiently an $a-a^{\prime}$ path $P^{\prime}$ in $G-\left\{b, b^{\prime}\right\}$ such that $G-V\left(P^{\prime}\right)$ has exactly two components. This will be used in Section 3 .

(2.6) Lemma. Let $\left(G, a, b, a^{\prime}, b^{\prime}\right)$ be a planar graph and suppose $G$ is $\left(4,\left\{a, a^{\prime}, b, b^{\prime}\right\}\right)$ connected. Then there exists an induced $a-a^{\prime}$ path $P^{\prime}$ in $G$ such that $G-V\left(P^{\prime}\right)$ has exactly two components $K$ and $K^{\prime}$ with $b \in V(K)$ and $b^{\prime} \in V\left(K^{\prime}\right)$. Moreover, such a path can be found in $O(|V(G)|+|E(G)|)$ time.

Proof. Take an embedding of $G$ in a closed disc such that $a, b, a^{\prime}, b^{\prime}$ occur on the boundary of the disc in the cyclic order listed. By Lemma (2.5), this can be done in $O(|V(G)|+$ $|E(G)|)$ time. Let $G^{\prime}:=\left(G-b^{\prime}\right)+\left\{a b, a^{\prime} b\right\}$.

We claim that $G^{\prime}$ is 2-connected. Suppose for a contradiction that $G^{\prime}$ is not 2connected. Let $x$ be a cut vertex of $G^{\prime}$. Since $G^{\prime}$ is $\left(4,\left\{a, a^{\prime}, b, b^{\prime}\right\}\right)$-connected, $G-\left\{b, b^{\prime}\right\}$ contains an $a-a^{\prime}$ path, and hence, $\left\{a, a^{\prime}, b\right\}$ is contained in a cycle in $G^{\prime}$. Therefore, $\left\{a, a^{\prime}, b\right\}$ is contained in an $x$-bridge of $G^{\prime}$, and $G^{\prime}$ has another $x$-bridge $B$ such that $(V(B)-\{x\}) \cap\left\{a, a^{\prime}, b\right\}=\emptyset$. Hence, $B-x$ is a component of $G-T$, where $T:=$ $\left\{x, b^{\prime}\right\}$ and $(V(B)-\{x\}) \cap\left\{a, a^{\prime}, b, b^{\prime}\right\}=\emptyset$, which contradicts the assumption that $G$ is $\left(4,\left\{a, a^{\prime}, b, b^{\prime}\right\}\right)$-connected.

Thus, we can assume that $a b, a^{\prime} b$ are in the cycle bounding the infinite face of $G^{\prime}$. Let $P^{\prime}$ be the $a-a^{\prime}$ subpath of this cycle which avoids $b$. Note that $N_{G}\left(b^{\prime}\right) \subseteq V\left(P^{\prime}\right)$ and $P^{\prime}$ can be computed in $O(|V(G)|+|E(G)|)$ time.

We claim that $G^{\prime}-V\left(P^{\prime}\right)$ is connected. Suppose for a contradiction that $G^{\prime}-V\left(P^{\prime}\right)$ is not connected. Let $\mathcal{K}$ be the set of components of $G^{\prime}-V\left(P^{\prime}\right)$ which do not contain $b$. For any $K \in \mathcal{K}$, let $u_{K}, u_{K}^{\prime} \in V\left(P^{\prime}\right)$ such that $N_{G^{\prime}}(K) \cap V\left(P^{\prime}\right) \subseteq V\left(P^{\prime}\left[u_{K}, u_{K}^{\prime}\right]\right)$ and $P^{\prime}\left[u_{K}, u_{K}^{\prime}\right]$ is minimal with respect to this property. If $|\mathcal{K}| \geq 2$, choose $K \in \mathcal{K}$ such that for any $K^{\prime} \neq K$, if $E\left(P\left[u_{K}, u_{K}^{\prime}\right]\right) \cap E\left(P\left[u_{K^{\prime}}, u_{K^{\prime}}^{\prime}\right]\right) \neq \emptyset$, then $P\left[u_{K}, u_{K}^{\prime}\right] \subseteq P\left[u_{K^{\prime}}, u_{K^{\prime}}^{\prime}\right]$; 
such a component must exist because of planarity. If $|\mathcal{K}|=1$, let $\mathcal{K}=\{K\}$. In either case, $N_{G}\left(P^{\prime}\left(u_{K}, u_{K}^{\prime}\right)\right) \subseteq V(K) \cup\left\{u_{K}, u_{K}^{\prime}, b^{\prime}\right\}$. Thus, $K \cup P^{\prime}\left(u_{K}, u_{K}^{\prime}\right)$ is contained in a component of $G-\left\{u_{K}, u_{K}^{\prime}, b^{\prime}\right\}$ that does not contain any vertex in $\left\{a, a^{\prime}, b, b^{\prime}\right\}$, which contradicts the assumption that $G$ is $\left(4,\left\{a, a^{\prime}, b, b^{\prime}\right\}\right)$-connected.

So $G^{\prime}-V\left(P^{\prime}\right)=G-\left(V\left(P^{\prime}\right) \cup\left\{b^{\prime}\right\}\right)$ is connected. Hence, $G-V\left(P^{\prime}\right)$ has exactly two components $K$ and $K^{\prime}$ with $b \in V(K)$ and $b^{\prime} \in V\left(K^{\prime}\right)$.

We now show that $P^{\prime}$ is an induced path in $G$. Suppose on the contrary that $P^{\prime}$ is not induced. Let $e=x y \in E(G)-E\left(P^{\prime}\right)$ with $x, y \in V\left(P^{\prime}\right)$. Then $V\left(P^{\prime}(x, y)\right) \neq \emptyset$. Moreover, by planarity $N_{G}\left(P^{\prime}(x, y)\right) \subseteq\left\{x, y, b^{\prime}\right\}$. Then $P^{\prime}(x, y)$ is contained in a component of $G-\left\{x, y, b^{\prime}\right\}$ that does not contain any vertex in $\left\{a, a^{\prime}, b, b^{\prime}\right\}$, which contradicts again the assumption that $G$ is $\left(4,\left\{a, a^{\prime}, b, b^{\prime}\right\}\right)$-connected.

Thus, $P^{\prime}$ is a path as required. Moreover, it is easy to see that such a path can be found in $O(|V(G)|+|E(G)|)$ time.

We conclude this section with another lemma which concerns non-separating induced paths in planar graphs.

(2.7) Lemma. Let $\left(G, a, a^{\prime}, b, b^{\prime}\right)$ be a planar graph, and suppose $G$ is $\left(4,\left\{a, a^{\prime}, b, b^{\prime}\right\}\right)$ connected and $G ¥ K_{1,4}$. Then there exists a non-separating induced $a-a^{\prime}$ path $P^{\prime}$ in $G$ such that $V\left(P^{\prime}\right) \cap\left\{b, b^{\prime}\right\}=\emptyset$. Moreover, such a path can be found in $O(|V(G)|+|E(G)|)$ time.

Proof. For convenience, let $S:=\left\{a, a^{\prime}, b, b^{\prime}\right\}$. Take an embedding of $G$ in a closed disc such that $a, a^{\prime}, b, b^{\prime}$ occur on the boundary of the disc in the cyclic order listed. By Lemma (2.5), this can be done in $O(|V(G)|+|E(G)|)$ time. Let $G^{\prime}:=G+\left\{a b^{\prime}, a^{\prime} b\right\}$.

We claim that $G^{\prime}$ is 2-connected. Suppose for a contradiction that $G^{\prime}$ is not 2connected. Let $x$ be a cut vertex of $G^{\prime}$. Since $G$ (and hence, $\left.G^{\prime}\right)$ is $(4, S)$-connected, it follows that any component of $G^{\prime}-x$ either contains only vertices in $S$, or contains at least one vertex in $V(G)-S$ and at least three vertices in $S$. Since $a^{\prime} b, a b^{\prime} \in E\left(G^{\prime}\right)$, $G^{\prime}-x$ cannot have both kinds of components. Therefore, every component of $G^{\prime}-x$ contains only vertices in $S$. Moreover, since $|V(G)| \geq 5, x \notin S$. But then, it is easy to see that $\left(G, a, a^{\prime}, b, b^{\prime}\right)$ must be isomorphic to $K_{1,4}$ with $x$ as the vertex of degree four, which contradicts the hypothesis. Hence, $G^{\prime}$ is 2 -connected.

Thus, we can assume that $a b^{\prime}, a^{\prime} b$ are in the cycle bounding the infinite face of $G^{\prime}$. Let $P^{\prime}$ be the $a-a^{\prime}$ subpath of this cycle which avoids $b$ and $b^{\prime}$. Note that $P^{\prime}$ is an $a-a^{\prime}$ path in $G$, and such a path can be found in $O(|V(G)|+|E(G)|)$ time.

We claim that $P^{\prime}$ is non-separating in $G$. Suppose for a contradiction that $G^{\prime}-V\left(P^{\prime}\right)$ is not connected. Note that $b$ and $b^{\prime}$ are contained in a component of $G-V\left(P^{\prime}\right)$. Let $\mathcal{K}$ be the set of components of $G^{\prime}-V\left(P^{\prime}\right)$ which contain neither $b$ nor $b^{\prime}$. For any $K \in \mathcal{K}$, let $u_{K}, u_{K}^{\prime} \in V\left(P^{\prime}\right)$ such that $N_{G^{\prime}}(K) \cap V\left(P^{\prime}\right) \subseteq V\left(P^{\prime}\left[u_{K}, u_{K}^{\prime}\right]\right)$ and $P^{\prime}\left[u_{K}, u_{K}^{\prime}\right]$ 
is minimal with respect to this property. If $|\mathcal{K}| \geq 2$, choose $K \in \mathcal{K}$ such that for any $K^{\prime} \neq K$, if $E\left(P\left[u_{K}, u_{K}^{\prime}\right]\right) \cap E\left(P\left[u_{K^{\prime}}, u_{K^{\prime}}^{\prime}\right]\right) \neq \emptyset$, then $P\left[u_{K}, u_{K}^{\prime}\right] \subseteq P\left[u_{K^{\prime}}, u_{K^{\prime}}^{\prime}\right]$; such a component must exist because of planarity. If $|\mathcal{K}|=1$, let $\mathcal{K}=\{K\}$. In either case, $N_{G}\left(P^{\prime}\left(u_{K}, u_{K}^{\prime}\right)\right) \subseteq V(K) \cup\left\{u_{K}, u_{K}^{\prime}\right\}$. Thus, $K \cup P^{\prime}\left(u_{K}, u_{K}^{\prime}\right)$ is contained in a component of $G-\left\{u_{K}, u_{K}^{\prime}\right\}$ that does not contain any vertex in $S$, which contradicts the assumption that $G$ is $(4, S)$-connected. Thus, $G-V\left(P^{\prime}\right)$ is connected.

Next we show that $P^{\prime}$ is an induced path in $G$. Suppose by contradiction that $P^{\prime}$ is not induced. Let $e=x y \in E(G)-E\left(P^{\prime}\right)$ such that $x, y \in V\left(P^{\prime}\right)$. Then $V\left(P^{\prime}(x, y)\right) \neq \emptyset$. Moreover, by planarity $N_{G}\left(P^{\prime}(x, y)\right) \subseteq\{x, y\}$. Then $P^{\prime}(x, y)$ is contained in a component of $G-\{x, y\}$ that does not contain any vertex in $S$, which contradicts again the assumption that $G$ is $(4, S)$-connected.

Thus, $P^{\prime}$ is a non-separating induced $a-a^{\prime}$ path in $G$ such that $V\left(P^{\prime}\right) \cap\left\{b, b^{\prime}\right\}=\emptyset$ as required.

\section{Internal chains}

In this section, we prove the following theorem, which will be used to construct internal chains in a non-separating chain decomposition. See Figure 3 for an illustration for the statement of the result. Recall that, for a graph $K$ and $u, v \in V(K), K-u v$ denotes the graph with vertex set $V(K)$ and edge set $E(K)-\{u v\}$ (note that $u v$ needs not be an edge of $K$ ).

(3.1) Theorem. Let $G$ be a 4-connected graph, let $F$ be a subgraph of $G$, and let $r \in V(F)$ such that $G_{F}:=G-(V(F)-\{r\})$ is 2-connected. Suppose that $G$ has a feasible $a-a^{\prime} F$-path $P$, that is, for some $a, a^{\prime} \in V(F), P$ is an $a-a^{\prime}$ path in $G-a a^{\prime}$ such that

(i) $V(P) \cap V(F)=\left\{a, a^{\prime}\right\}$ and $P$ is an induced path in $G-a a^{\prime}$,

(ii) $P\left(a, a^{\prime}\right)$ is a non-separating path in $G_{F}$,

(iii) $r$ is contained in a nontrivial block $B_{P}$ of $G_{F}-V\left(P\left(a, a^{\prime}\right)\right)$, and

(iv) if $r \in\left\{a, a^{\prime}\right\}$, then $r$ is not a cut vertex of $G_{F}-V\left(P\left(a, a^{\prime}\right)\right)$.

Then there exists a good $F$-chain $H$ in $G$ such that $G_{F}-I(H)$ is 2-connected, $G[V(F) \cup$ $I(H)]$ is 2-connected, and $B_{P} \subseteq G_{F}-I(H)$. Moreover, such a chain can be found in $O(|V(G)||E(G)|)$ time.

Remark. Condition (iv) in Theorem (3.1) is necessary for a technical reason, and the reader may want to assume in a first reading that $r \notin\left\{a, a^{\prime}\right\}$ to familiarize with the proof. 


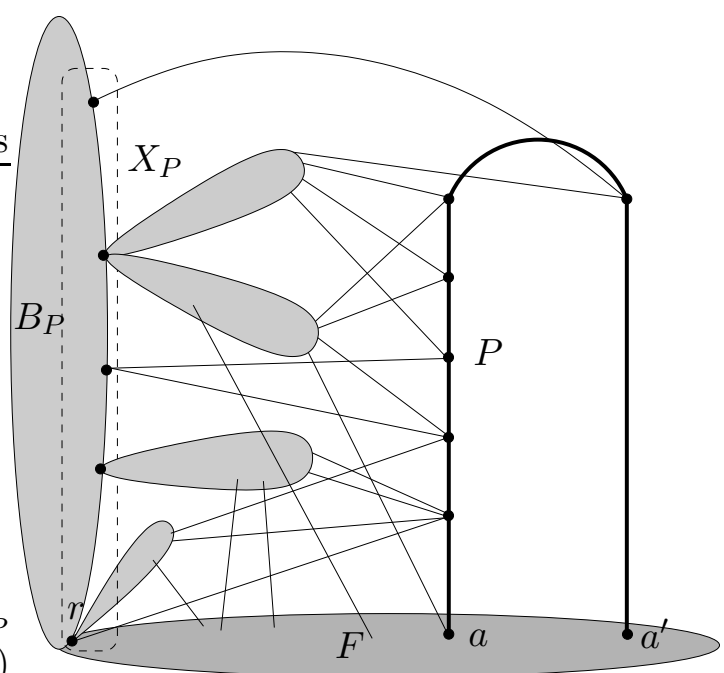

G

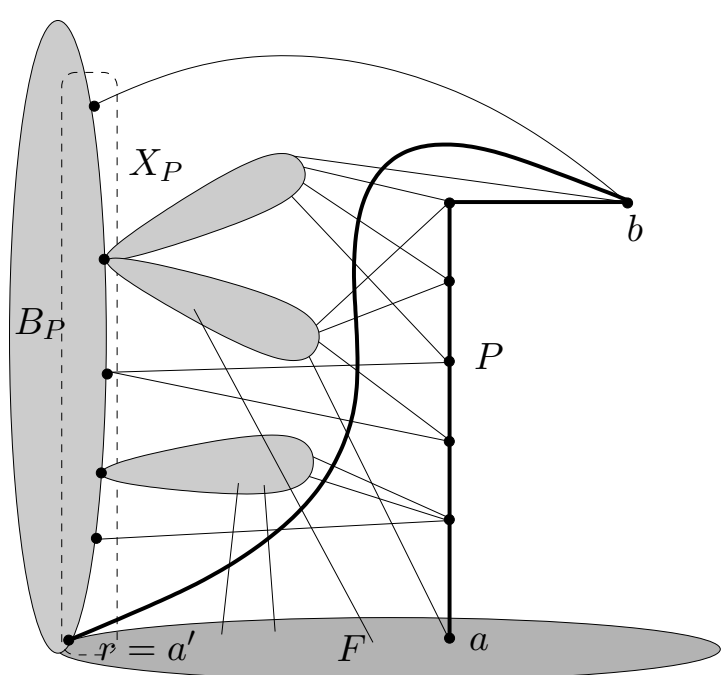

G

Figure 3: Illustration for (3.1) and (3.2): one with $r \notin\left\{a, a^{\prime}\right\}$ and the other with $r \in\left\{a, a^{\prime}\right\}$.

Throughout the rest of this section, we fix the following notation.

(3.2) Notation and definitions. Let $G$ be a 4-connected graph, let $F$ be a subgraph of $G$, and let $r \in V(F)$ such that $G_{F}:=G-(V(F)-\{r\})$ is 2-connected. Suppose $G$ has a feasible $a-a^{\prime} F$-path $P$ and $r$ is contained in a nontrivial block $B_{P}$ of $G_{F}-V\left(P\left(a, a^{\prime}\right)\right)$.

Let $\mathcal{P}_{P}$ be the set of feasible $F$-paths $P^{\prime}$ (with ends, say $u, u^{\prime}$ ) in $G$ such that $B_{P} \subseteq$ $G_{F}-V\left(P^{\prime}\left(u, u^{\prime}\right)\right)$. For each $P^{\prime} \in \mathcal{P}_{P}$ with ends, say $u, u^{\prime}$, let $B_{P^{\prime}}$ denote the block of $G_{F}-V\left(P^{\prime}\left(u, u^{\prime}\right)\right)$ which contains $B_{P}$. We say that $P^{\prime} \in \mathcal{P}_{P}$ is a $B_{P}$-augmenting path if $\left|V\left(B_{P}\right)\right|<\left|V\left(B_{P^{\prime}}\right)\right|$.

We will describe an algorithm for finding a good $F$-chain as required in (3.1). The idea of the algorithm is roughly the following. At the beginning of each iteration we have vertices $a, a^{\prime} \in V(F)$ and a feasible $a-a^{\prime} F$-path $P$ in $G$. The algorithm iteratively tries to

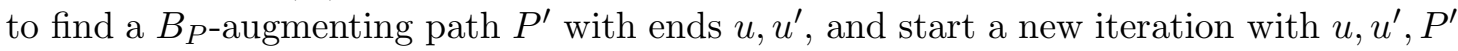
as $a, a^{\prime}, P$ respectively. Note that $r, u, u^{\prime}, P^{\prime}, F$ and $G$ (as $r, a, a^{\prime}, P, F$ and $G$ respectively) satisfy the hypotheses of (3.1) with $B_{P}$ enlarged to $B_{P^{\prime}}$. When the algorithm does not find such a path, it finds a good $F$-chain as required in (3.1).

The next lemma says that (assuming $G$ has a feasible $a-a^{\prime} F$-path $P$ ) one can find in $O(|V(G)|+|E(G)|)$ time a feasible $u$ - $u^{\prime} F$-path $P^{\prime}$ such that $\left|V\left(P^{\prime}\right)\right|=3$ or $N_{G}\left(P^{\prime}\left(u, u^{\prime}\right)\right) \cap V(F) \subseteq\left\{u, u^{\prime}\right\} \cup\{r\}$. The latter condition is equivalent to requiring that 
$N_{G}\left(P^{\prime}\left(u, u^{\prime}\right)\right) \cap V(F)=\left\{u, u^{\prime}\right\}$ when $r \in\left\{u, u^{\prime}\right\}$, or $N_{G}\left(P^{\prime}\left(u, u^{\prime}\right)\right) \cap V(F) \subseteq\left\{u, u^{\prime}, r\right\}$ when $r \notin\left\{u, u^{\prime}\right\}$ (see Figure 3).

(3.3) Lemma. There exist $u, u^{\prime} \in V(F)$ and a feasible $u-u^{\prime} F$-path $P^{\prime}$ such that

(1) $\left|V\left(P^{\prime}\right)\right|=3$ or $N_{G}\left(P\left(u, u^{\prime}\right)\right) \cap V(F) \subseteq\left\{u, u^{\prime}\right\} \cup\{r\}$, and

(2) $B_{P} \subseteq B_{P^{\prime}}$

Moreover, such a path can be found in $O(|V(G)|+|E(G)|)$ time.

Proof. If either $|V(P)|=3$ or $N_{G}\left(P\left(a, a^{\prime}\right)\right) \cap V(F) \subseteq\left\{a, a^{\prime}\right\} \cup\{r\}$, then the result follows with $P^{\prime}:=P$.

Thus, assume that $|V(P)| \geq 4$ and $\left(N_{G}\left(P\left(a, a^{\prime}\right)\right) \cap V(F)\right)-\left(\left\{a, a^{\prime}\right\} \cup\{r\}\right) \neq \emptyset$. By symmetry, we may assume that $a \neq r$. Let $v \in V\left(P\left(a, a^{\prime}\right)\right)$ such that $v$ has a neighbor in $V(F)-\left(\left\{a, a^{\prime}\right\} \cup\{r\}\right)$, and subject to this, $P[a, v]$ is minimal. If $v$ has two neighbors in $V(F)-\{r, a\}$, say $u$ and $u^{\prime}$, let $P^{\prime}:=\left(u, v, u^{\prime}\right)$. In this case, (1) holds with $\left|V\left(P^{\prime}\right)\right|=3$. If $v$ has exactly one neighbor in $V(F)-\{r, a\}$, say $u$, then let $P^{\prime}:=P[a, v]+\{u, v u\}$ and $u^{\prime}:=a$. Note that in both cases $r \notin\left\{u, u^{\prime}\right\}$. By the choice of $v, N_{G}\left(P\left(u, u^{\prime}\right)\right) \cap V(F) \subseteq\left\{u, u^{\prime}\right\} \cup\{r\}$, and hence, (1) holds. Moreover, since $G_{F}-V\left(P\left(a, a^{\prime}\right)\right) \subseteq G_{F}-V\left(P^{\prime}\left(u, u^{\prime}\right)\right)$, we have $B_{P} \subseteq G_{F}-V\left(P^{\prime}\left(u, u^{\prime}\right)\right)$, and hence, (2) holds.

Finally, we show that $P^{\prime}$ is a feasible $u-u^{\prime} F$-path. Since $P$ is induced in $G-a a^{\prime}$, $P^{\prime}$ is induced in $G-u u^{\prime}$. Clearly $V\left(P^{\prime}\right) \cap V(F)=\left\{u, u^{\prime}\right\}$, so (i) of (3.1) holds. Since $G_{F}$ is 2-connected and $P\left(a, a^{\prime}\right)$ is an induced path in $G_{F}-a a^{\prime}$, if $V\left(P\left(v, a^{\prime}\right)\right) \neq \emptyset$, then $N_{G_{F}}\left(P\left(v, a^{\prime}\right)\right) \cap\left(V\left(G_{F}\right)-V\left(P\left(a, a^{\prime}\right)\right)\right) \neq \emptyset$. Thus, since $G_{F}-V\left(P\left(a, a^{\prime}\right)\right)$ is connected, $P^{\prime}\left(u, u^{\prime}\right)$ is non-separating in $G_{F}$, so (ii) of (3.1) holds. Also, $r$ is contained in a nontrivial block of $G_{F}-V\left(P^{\prime}\left(u, u^{\prime}\right)\right)$, because $r \in B_{P} \subseteq G_{F}-V\left(P^{\prime}\left(u, u^{\prime}\right)\right)$, so (iii) of (3.1) holds. Since $r \notin\left\{u, u^{\prime}\right\}$, we do not need to verify (iv) in (3.1).

Therefore, $P^{\prime}$ is a feasible $F$-path as required, and it is not hard to see that such a path $P^{\prime}$ can be found in $O(|V(G)|+|E(G)|)$ time.

(3.4) Assumption. Using Lemma (3.3), we can pre-process a feasible $F$-path at the beginning of each iteration (in $O(|V(G)|+|E(G)|)$ time). Henceforth, we may assume that for the (current) feasible $F$-path $P,|V(P)|=3$ or $N_{G}\left(P\left(a, a^{\prime}\right)\right) \cap V(F) \subseteq\left\{a, a^{\prime}\right\} \cup\{r\}$. We may also assume that $G_{F}-V\left(P\left(a, a^{\prime}\right)\right)$ is not 2-connected, for otherwise $H:=P$ gives a $F$-chain as required in (3.1): $H$ is an up $F$-chain (where each of its blocks is trivial), or $H$ is an elementary $F$-chain. Moreover, $G_{F}-I(H)=G_{F}-V\left(P\left(a, a^{\prime}\right)\right)$ is 2-connected.

(3.5) Notation. Let $X_{P}:=N_{G_{F}}\left(G_{F}-V\left(B_{P}\right)\right)$. For each $B_{P}$-bridge $B$ of $G_{F}-$ $V\left(P\left(a, a^{\prime}\right)\right)$, let $r_{B}$ denote the unique vertex in $V(B) \cap V\left(B_{P}\right)$. Note that $r_{B} \in X_{P}$. Also, if $r \in\left\{a, a^{\prime}\right\}$, then $r \in X_{P}$. See Figure 3 for an illustration. 
Remark. Note that since $G_{F}$ is 2-connected, we have $\left|X_{P}\right| \geq 2$. Moreover, if $B$ is a $B_{P}$-bridge of $G_{F}-V\left(P\left(a, a^{\prime}\right)\right)$ then $V(B)-\left\{r_{B}\right\}$ has a neighbor in $V\left(P\left(a, a^{\prime}\right)\right)$.

The next lemma shows that if, for every $B_{P}$-bridge $B$ of $G_{F}-V\left(P\left(a, a^{\prime}\right)\right), N_{G}(B-$ $\left.r_{B}\right) \subseteq V(P)$, then one can find efficiently a good $F$-chain (in fact, an up $F$-chain) $H$ as required in (3.1) by invoking Theorem (1.7).

(3.6) Lemma. Suppose that for every $B_{P}$-bridge $B$ of $G_{F}-V\left(P\left(a, a^{\prime}\right)\right), N_{G}\left(B-r_{B}\right) \subseteq$ $V(P)$. Then there exists an $a-a^{\prime}$ up $F$-chain $H$ in $G$ such that $G_{F}-I(H)$ is 2-connected, $G[V(F) \cup I(H)]$ is 2-connected, and $B_{P} \subseteq G_{F}-I(H)$. Moreover, such a chain can be found in $O(|V(G)||E(G)|)$ time.

Proof. Suppose first that $r \notin\left\{a, a^{\prime}\right\}$ (see Figure 3). Let $G^{\prime}$ be the graph obtained from $G_{F}$ by adding $\left\{a, a^{\prime}\right\}$ and the edges of $G$ from $\left\{a, a^{\prime}\right\}$ to $V\left(G_{F}\right)-\{r\}$. Note that $P$ is a non-separating induced $a-a^{\prime}$ path in $G^{\prime}$. Note also that $B_{P}$ is a nontrivial block of $G^{\prime}-V(P)$. Let $X_{P}^{\prime}=N_{G^{\prime}}\left(G^{\prime}-V\left(B_{P}\right)\right)$.

We claim that $G^{\prime}-\left(V\left(B_{P}\right)-X_{P}^{\prime}\right)$ is $\left(4, X_{P}^{\prime} \cup\left\{a, a^{\prime}\right\}\right)$-connected. For convenience, let $K:=G^{\prime}-\left(V\left(B_{P}\right)-X_{P}^{\prime}\right)$. Since for any $B_{P}$-bridge $B$ of $G^{\prime}-V(P)=G_{F}-V\left(P\left(a, a^{\prime}\right)\right)$, $V(B)-\left\{r_{B}\right\}$ has a neighbor in $V\left(P\left(a, a^{\prime}\right)\right)$, it follows that $K$ is connected and $K-\left(X_{P}^{\prime} \cup\right.$ $\left.\left\{a, a^{\prime}\right\}\right)$ is a component of $G-\left(X_{P}^{\prime} \cup\left\{a, a^{\prime}\right\}\right)$. Hence, because $G$ is 4-connected, $K$ is $\left(4, X_{P}^{\prime} \cup\left\{a, a^{\prime}\right\}\right)$-connected.

Thus, the hypotheses of (1.7) are satisfied with $G^{\prime}, a, a^{\prime}, P, B_{P}, X_{P}^{\prime}$ as $G, a, b, P, B_{P}, X_{P}$ respectively. Hence, there exists a planar $a-a^{\prime} F$-chain $H$ in $G^{\prime}$ such that $G^{\prime}-V(H)=G_{F}-I(H)$ is 2-connected and $B_{P} \subseteq G^{\prime}-V(H)=G_{F}-I(H)$. Moreover, such a chain can be found in $O\left(\left|V\left(G^{\prime}\right)\right|\left|E\left(G^{\prime}\right)\right|\right)$ time (and hence, in $O(|V(G)||E(G)|)$ time). Note also that $H$ is an up $F$-chain in $G$, and hence, $G[V(F) \cup I(H)]$ is 2-connected, so the result follows.

Now suppose that $r \in\left\{a, a^{\prime}\right\}$, and without loss of generality, let $r=a^{\prime}$ (see Figure 3). Let $b$ be the neighbor of $r$ in $P$. Let $G^{\prime}$ be the graph obtained from $G_{F}$ by adding $a$ and the edges of $G$ from $a$ to $V\left(G_{F}\right)-\{r\}$. Note that $b \in V\left(G^{\prime}\right)$, and $P[a, b]$ is a nonseparating induced path in $G^{\prime}$. Note also that $B_{P}$ is a nontrivial block of $G^{\prime}-V(P[a, b])=$ $G_{F}-V(P(a, r))$. Let $X_{P}^{\prime}=N_{G^{\prime}}\left(G^{\prime}-V\left(B_{P}\right)\right)$. Since $P$ is a feasible $a-r F$-path, $r$ is not a cut vertex of $G^{\prime}-V(P[a, b])=G_{F}-V\left(P(a, r)\right.$ (in particular, there is no $B_{P}$-bridge in $G^{\prime}-V(P[a, b])$ containing $\left.r\right)$.

We claim that $G^{\prime}-\left(V\left(B_{P}\right)-X_{P}^{\prime}\right)$ is $\left(4, X_{P}^{\prime} \cup\{a, b\}\right)$-connected. For convenience, let $K:=G^{\prime}-\left(V\left(B_{P}\right)-X_{P}^{\prime}\right)$. Since for any $B_{P}$-bridge $B$ of $G_{F}-V(P(a, r)), V(B)-$ $\left\{r_{B}\right\}$ has at least two neighbors in $V(P(a, r))$ (because $G$ is 4-connected), it follows that $V(B)-\left\{r_{B}\right\}$ has at least one neighbor in $V(P(a, b))$. Hence, $K$ is connected and $K-\left(X_{P}^{\prime} \cup\{a, b\}\right)$ is a component of $G-\left(X_{P}^{\prime} \cup\{a, b\}\right)$. Since $G$ is 4-connected, $K$ is $\left(4, X_{P}^{\prime} \cup\{a, b\}\right)$-connected. 
Thus, the hypotheses of (1.7) are satisfied with $G^{\prime}, a, b, P[a, b], B_{P}, X_{P}^{\prime}$ as $G, a, b, P, B_{P}, X_{P}$ respectively. Hence, there exists a planar $a-b F$-chain $H^{\prime}$ in $G^{\prime}$ such that $G^{\prime}-V\left(H^{\prime}\right)$ is 2-connected and $B_{P} \subseteq G^{\prime}-V\left(H^{\prime}\right)$. Moreover, such a chain can be found in $O\left(\left|V\left(G^{\prime}\right)\right|\left|E\left(G^{\prime}\right)\right|\right)$ time (and hence, $O(|V(G)||E(G)|)$ time). Since $b$ is the only neighbor of $r$ in $V(P)-\{a, r\}, r \notin N_{G}\left(V\left(H^{\prime}\right)-\{a, b\}\right)$. Thus, $H:=H^{\prime}+r b$ is an up a-r $F$-chain in $G$ (recall $a^{\prime}=r$ ), so $G[V(F) \cup I(H)]$ is 2-connected. Note also that $G_{F}-I(H)=G^{\prime}-V\left(H^{\prime}\right)$ is 2-connected, and hence, the result follows.

Next, we show that if $\left|X_{P}\right|=2$, then one can find efficiently either a $B_{P}$-augmenting path or a good $F$-chain as required in (3.1).

(3.7) Lemma. Suppose that $\left|X_{P}\right|=2$ and let $v, v^{\prime}$ be the vertices in $X_{P}$. Then exactly one of the following holds:

(1) there exists a $B_{P}$-augmenting path, or

(2) $H:=\left(G_{F}-\left(V\left(B_{P}\right)-X_{P}\right)\right)-v v^{\prime}$ is a down $v$-v' $F$-chain in $G$ such that $G_{F}-I(H)$ is 2-connected and $G[V(F) \cup I(H)]$ is 2-connected.

Moreover, one can in $O(|V(G)|+|E(G)|)$ time either find a path as in (1), or certify that (2) holds.

Proof. Let $H:=\left(G_{F}-\left(V\left(B_{P}\right)-X_{P}\right)\right)-v v^{\prime}$. Since $G_{F}$ is 2-connected and $X_{P}=\left\{v, v^{\prime}\right\}$, $H$ is a $v-v^{\prime}$ chain in $G$ and $N_{G}\left(H-\left\{v, v^{\prime}\right\}\right) \subseteq V(F-r) \cup\left\{v, v^{\prime}\right\}$. See Figure 4 for an example. Let $H:=v_{0} B_{1} v_{1} \ldots v_{k-1} B_{k} v_{k}$, where $v_{0}=v$ and $v_{k}=v^{\prime}$. This decomposition of $H$ into blocks can be computed in $O(|V(G)|+|E(G)|)$ time. If every block of $H$ is trivial, then $H$ is a down $F$-chain, $G_{F}-I(H)=B_{P}$ is 2-connnected, and $G[V(F) \cup I(H)]$ is 2-connected, so (2) holds.

Thus, we may assume that $H$ contains a nontrivial block. For each nontrivial block $B_{i}$, let $S_{i}:=V(F-r) \cap N_{G}\left(B_{i}-\left\{v_{i-1}, v_{i}\right\}\right)$ and let $G_{i}$ be the graph obtained from $B_{i}$ by adding $S_{i}$ and the edges of $G$ from $S_{i}$ to $V\left(B_{i}\right)-\left\{v_{i-1}, v_{i}\right\}$. Note that $G_{i}-S_{i}=B_{i}$ is 2-connected and $B_{i}-\left\{v_{i-1}, v_{i}\right\}$ is a union of components of $G-\left(S_{i} \cup\left\{v_{i-1}, v_{i}\right\}\right)$. Because $G$ is 4-connected, $G_{i}$ is $\left(4, S_{i} \cup\left\{v_{i-1}, v_{i}\right\}\right)$-connected, and every component of $B_{i}-\left\{v_{i-1}, v_{i}\right\}$ has at least two neighbors in $S_{i}$. Thus, the hypotheses of (2.3) are satisfied with $G_{i}, S_{i}, v_{i-1}, v_{i}$ as $G, S, b, b^{\prime}$, respectively.

Hence, either (a) there exist $u_{i}, u_{i}^{\prime} \in S_{i}$ and an induced $u_{i}-u_{i}^{\prime}$ path $P_{i}^{\prime}$ in $G_{i}$ such that $V\left(P_{i}\right) \cap\left\{v_{i-1}, v_{i}\right\}=\emptyset, V\left(P_{i}\right) \cap S_{i}=\left\{u_{i}, u_{i}^{\prime}\right\}$ and $G_{i}-\left(V\left(P_{i}\right) \cup S_{i}\right)$ is connected, or (b) $\left|S_{i}\right|=2$ and the elements of $S_{i}$ can be labeled as $u_{i}, u_{i}^{\prime}$ such that $\left(G_{i}, v_{i-1}, u_{i}, v_{i}, u_{i}^{\prime}\right)$ is planar. Moreover, one can in $O\left(\left|V\left(G_{i}\right)\right|+\left|E\left(G_{i}\right)\right|\right)$ time find a path as in (a) or certify that (b) holds. If (a) holds for some nontrivial block $B_{i}$, then $P_{i}^{\prime}$ is a $B_{P}$-augmenting path for the following reasons: (i)-(iii) of (3.1) hold, $r \notin\left\{u, u^{\prime}\right\}$ (so (iv) of (3.1) holds), 


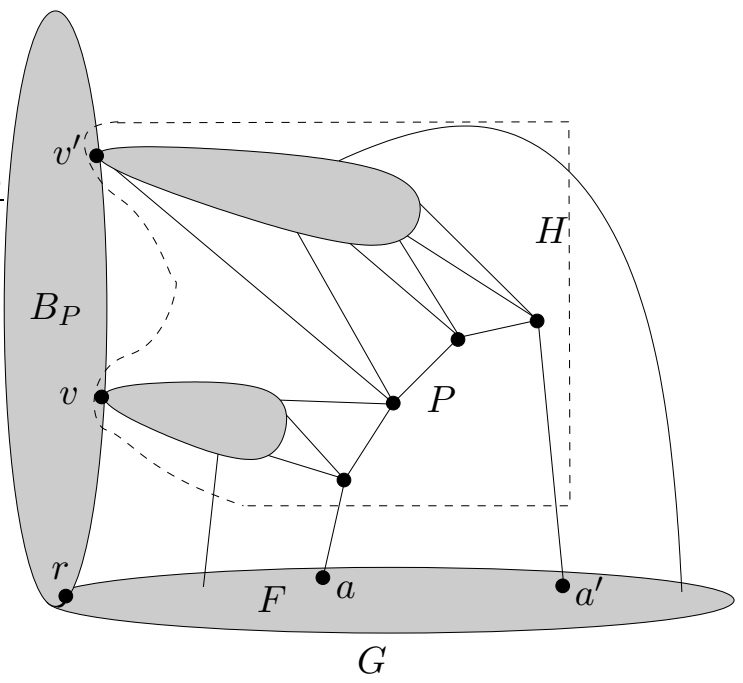

Figure 4: Graph $H$ in the proof of (3.7).

and there exists a $v-v^{\prime}$ path contained in $H-V\left(P_{i}^{\prime}\left(u_{i}, u_{i}^{\prime}\right)\right)$ (so $B_{P}$ is properly contained in $B_{P^{\prime}}$ ). If (b) holds for every nontrivial block $B_{i}$, then $H$ is clearly a down $F$-chain, $G[V(\stackrel{l}{F}) \cup I(H)]$ is 2-connected (because $G$ is 4 -connected, and so $G_{i}-\left\{v_{i-1}, v_{i}\right\}$ is a $u_{i}-u_{i}^{\prime}$ chain), and $G_{F}-I(H)$ is 2-connected.

One can verify if either (1) or (2) holds in $O(|V(G)|+|E(G)|)$ time, because if (b) holds for a nontrivial block $B_{i}$, then $\left|V\left(G_{i}\right)\right|+\left|E\left(G_{i}\right)\right|=O\left(\left|V\left(B_{i}\right)\right|+\left|E\left(B_{i}\right)\right|\right)$, and if (a) holds for some $G_{i}$, then $\left|V\left(G_{i}\right)\right|+\left|E\left(G_{i}\right)\right|=O(|V(G)|+|E(G)|)$. In the latter case, we find a $B_{P}$-augmenting path and we stop. Thus, this verification can be carried over in $O(|V(G)|+|E(G)|)$ time.

The following lemma shows that if $\left|X_{P}\right| \geq 3$ and $|V(P)|=3$, then one can find efficiently a $B_{P}$-augmenting path.

(3.8) Lemma. Suppose that $\left|X_{P}\right| \geq 3$ and $|V(P)|=3$. Then exactly one of the following holds:

(1) there exists a $B_{P}$-augmenting path, or

(2) $P$ is an elementary $F$-chain in $G$ such that $G_{F}-I(P)$ is 2-connected and $G[V(F) \cup$ $I(P)]$ is 2-connected.

Moreover, one can in $O(|V(G)|+|E(G)|)$ time either find a path as in (1), or certify that (2) holds. 
Proof. If $G_{F}-V\left(P\left(a, a^{\prime}\right)\right)$ is 2-connected, then $P$ is an elementary $F$-chain in $G, G_{F}-$ $I(P)$ is 2-connected, and $G[V(F) \cup I(P)]$ is 2-connected, so (2) holds. Note this can be checked in $O(|V(G)|+|E(G)|)$ time.

So we may assume that $G_{F}-V\left(P\left(a, a^{\prime}\right)\right)$ is not 2-connected. Let $K$ be a $B_{P}$-bridge of $G_{F}-V\left(P\left(a, a^{\prime}\right)\right)$ and let $v$ denote the unique vertex in $V\left(P\left(a, a^{\prime}\right)\right)$. If $K$ is 2-connected, then let $B:=K$ and let $b:=r_{K}$. Otherwise let $B$ be an endblock of $K$, and let $b$ denote the cut vertex of $K$ contained in $V(B)$. Since $G_{F}$ is 2-connected, $v \in N_{G}(B-b)$. Note that $B$ can be computed in $O(|V(G)|+|E(G)|)$ time.

First, suppose that $B$ is trivial, and let $w$ be the unique vertex in $V(B-b)$. Since $G$ is 4-connected, $w$ has at least three neighbors in $V(F-r) \cup\{v\}$, and hence, it has two neighbors $u, u^{\prime}$ in $V(F-r)$. Let $P^{\prime}:=\left(u, w, u^{\prime}\right)$. We claim that $P^{\prime}$ is a feasible $F$-path. Clearly, $P^{\prime}$ is an induced path in $G-u u^{\prime}$ and $V\left(P^{\prime}\right) \cap V(F)=\left\{u, u^{\prime}\right\}$. Since $G_{F}$ is 2-connected, $G_{F}-V\left(P^{\prime}\left(u, u^{\prime}\right)\right)=G_{F}-w$ is connected. Thus, $P^{\prime}\left(u, u^{\prime}\right)$ is non-separating in $G_{F}$. Also $r \in V\left(B_{P}\right)$ and $B_{P} \subseteq G_{F}-V\left(P^{\prime}\left(u, u^{\prime}\right)\right)$. Therefore, since $r \notin\left\{u, u^{\prime}\right\}, P^{\prime}$ is a feasible $F$-path. Since $\left|X_{P}\right| \geq 3$, there exists a path (containing $v$ ) with ends in $X_{P}-\left\{r_{B}\right\}$ which is internally disjoint from $V\left(B_{P}\right) \cup V(B)$. Therefore, $B_{P}$ is properly contained in $B_{P^{\prime}}$, and hence, $P^{\prime}$ is a $B_{P^{-a u g m e n t i n g ~ p a t h . ~}}$

Thus, we may assume that $B$ is nontrivial, so $B$ is 2-connected. Let $S:=N_{G}(B-$ $b)-\{b, v\}$ and let $G^{\prime}$ be obtained from $B$ by adding $S$ and the edges of $G$ from $S$ to $V(B)-\{b\}$. Note that $S \subseteq V(F-r)$ and $G^{\prime}-S=B$ is 2-connected. Since $G$ is 4-connected, $G\left[V\left(G^{\prime}\right) \cup\{v\}\right]$ is $(4, S \cup\{b, v\})$-connected, and hence, $G^{\prime}$ is $(3, S \cup\{b\})$ connected. By Lemma (2.4) (with $G^{\prime}, b, S$ as $G, b, S$, respectively) there exist $u, u^{\prime} \in S$ and an induced $u-u^{\prime}$ path $P^{\prime}$ in $G^{\prime}$ such that $V\left(P^{\prime}\right) \cap\{b\}=\emptyset, V\left(P^{\prime}\right) \cap S=\left\{u, u^{\prime}\right\}$ and $G^{\prime}-\left(V\left(P^{\prime}\right) \cup S\right)$ is connected. Moreover, such a path can be found in $O\left(\left|V\left(G^{\prime}\right)\right|+\left|E\left(G^{\prime}\right)\right|\right)$ time (and hence, in $O(|V(G)|+|E(G)|)$ time).

We claim that $P^{\prime}$ is a feasible $F$-path. Clearly, $P^{\prime}$ is an induced path in $G-u u^{\prime}$ and $V\left(P^{\prime}\right) \cap V(F)=\left\{u, u^{\prime}\right\}$. Since $G^{\prime}-\left(V\left(P^{\prime}\right) \cup S\right)=B-V\left(P^{\prime}\left(u, u^{\prime}\right)\right)$ is connected, and $b \notin V\left(P^{\prime}\right)$, we have that $G_{F}-V\left(P^{\prime}\left(u, u^{\prime}\right)\right)$ is connected. Thus, $P^{\prime}\left(u, u^{\prime}\right)$ is non-separating in $G_{F}$. Also $r \in V\left(B_{P}\right)$ and $B_{P} \subseteq G_{F}-V\left(P^{\prime}\left(u, u^{\prime}\right)\right)$. Since $r \notin S, r \notin\left\{u, u^{\prime}\right\}$, so $P^{\prime}$ is a feasible $F$-path. Furthermore, since $\left|X_{P}\right| \geq 3$, there exists a path (containing $v$ ) with ends in $X_{P}-\left\{r_{B}\right\}$ which is internally disjoint from $V\left(B_{P}\right) \cup V(B)$. Therefore, $B_{P}$ is

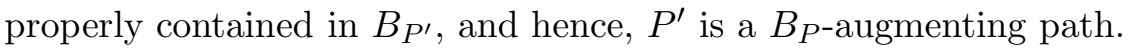

By (3.6), (3.7) and (3.8), we only need to deal with the case where $\left|X_{P}\right| \geq 3,|V(P)| \geq$ 4 , and for some $B_{P}$-bridge $B$ of $G_{F}-V\left(P\left(a, a^{\prime}\right)\right), B-r_{B}$ has a neighbor in $V(F-r)-$ $\left\{a, a^{\prime}\right\}$. Our aim is to prove that we can find either a $B_{P}$-augmenting path or a triangle $F$-chain $H$ such that $G_{F}-I(H)$ is 2-connected. In order to do this, we need to introduce some notation and auxiliary results. 
(3.9) Notation. For any $x, y \in V(P)$, we denote $x \leq y$ if $x \in V(P[a, y])$. If $x \leq y$ and $x \neq y$, then we write $x<y$. In this case, we say that $x$ is lower than $y$, or $y$ is higher than $x$.

Let $X_{P}:=\left\{r_{1}, \ldots, r_{p}\right\}$. For each $i, 1 \leq i \leq p$, if $r_{i}$ is a cut vertex of $G_{F}-V\left(P\left(a, a^{\prime}\right)\right)$, then let $V_{i}:=\bigcup V(B)$, where the union is taken over all the $B_{P}$-bridges $B$ of $G_{F}-$ $V\left(P\left(a, a^{\prime}\right)\right)$ with $r_{B}=r_{i}$; if $r_{i}$ is not a cut vertex of $G_{F}-V\left(P\left(a, a^{\prime}\right)\right)$, then let $V_{i}:=\left\{r_{i}\right\}$.

For each $i$ such that $V_{i} \neq\left\{r_{i}\right\}$, let $x_{i}, y_{i} \in V(P)$ with $x_{i} \leq y_{i}$ such that $G$ has an edge from $x_{i}$ (respectively, $y_{i}$ ) to $V_{i}$ which is not an edge from $\left\{a, a^{\prime}\right\}$ to $r_{i}$, and subject to this, $P\left[x_{i}, y_{i}\right]$ is maximal. Note that we may have $x_{i}=a$ or $y_{i}=a^{\prime}$, but $r \notin\left\{x_{i}, y_{i}\right\}$ because $B_{P}$ is a block of $G_{F}-V\left(P\left(a, a^{\prime}\right)\right)$.

Let $P_{i}:=P\left[x_{i}, y_{i}\right]$ and let $H_{i}$ be the graph obtained from $G\left[V_{i} \cup V\left(P_{i}\right)\right]$ by removing all edges from $\left\{a, a^{\prime}\right\}$ to $r_{i}$. Let $\mathcal{H}:=\left\{H_{i}: 1 \leq i \leq p, V_{i} \neq\left\{r_{i}\right\}\right\}$. We say that $H_{i} \in \mathcal{H}$ is adjacent to $F$ if $N_{G}\left(V_{i}-\left\{r_{i}\right\}\right) \cap\left(V(F-r)-\left\{a, a^{\prime}\right\}\right) \neq \emptyset$. See Figure 5 for an example.
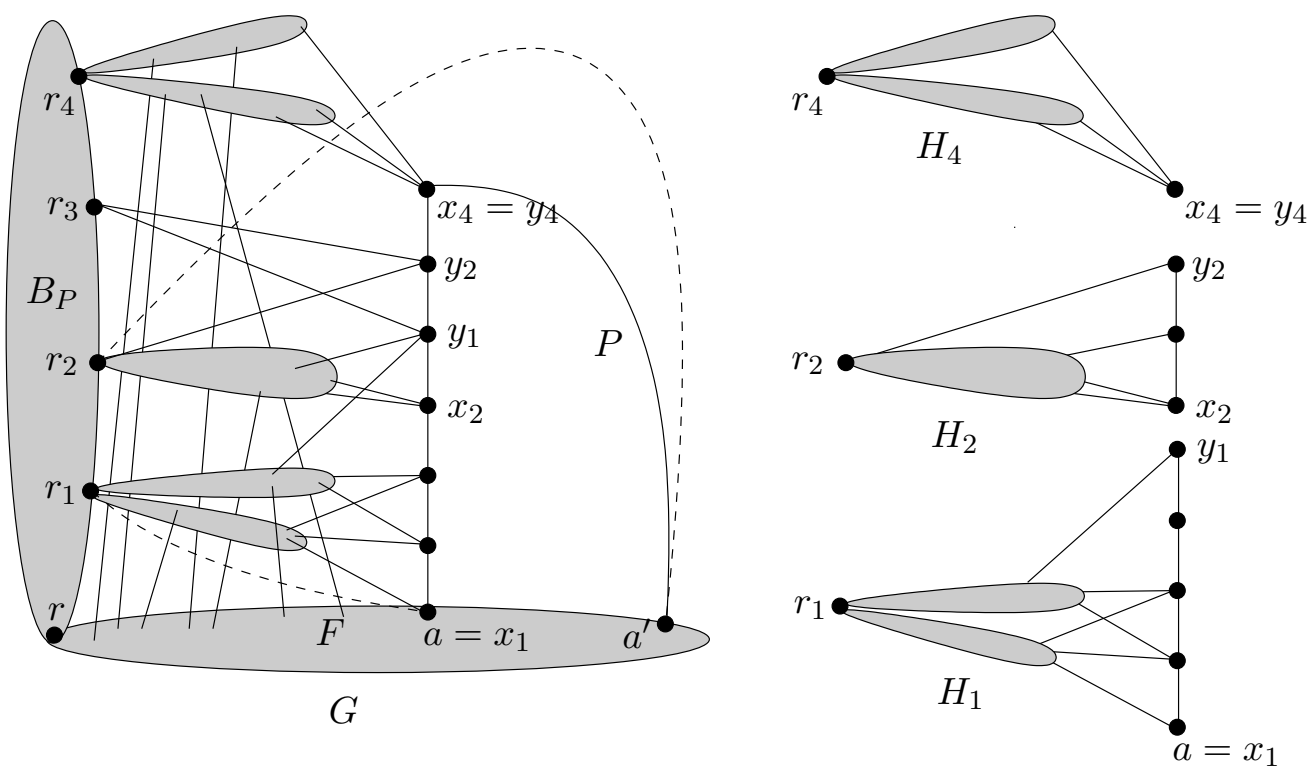

Figure 5: Example for (3.9) with $X_{P}=\left\{r_{1}, r_{2}, r_{3}, r_{4}\right\}$. Note that the edges $r_{1} a, r_{2} a^{\prime}$ are not contained in any $H_{i}$.

(3.10) Lemma. Every $H_{i} \in \mathcal{H}$ is an $r_{i}-x_{i}$ (and also an $r_{i}-y_{i}$ ) chain. Moreover, no vertex of $P_{i}$ is a cut vertex of $H_{i}$, and $P_{i}$ is contained in an endblock of $H_{i}$.

Proof. Since $G\left[V_{i}\right]=H_{i}-V\left(P_{i}\right)$ is connected and because $H_{i}$ have edges from both $x_{i}$ and $y_{i}$ to $V_{i}$, no vertex of $P_{i}$ is a cut vertex of $H_{i}$, and hence, $P_{i}$ is contained in a block 
of $H_{i}$. We claim that if $B$ is an endblock of $H_{i}$, then $r_{i} \in V(B)$ or $V\left(P_{i}\right) \subseteq V(B)$ (and hence, we have (3.10)). Suppose for a contradiction that $B$ is an endblock of $H_{i}$ and $B$ contains neither $r_{i}$ nor any vertex in $V\left(P_{i}\right)$. Let $v$ be the cut vertex of $H_{i}$ contained in $V(B)$. Then $B-v$ is a component of $G_{F}-v$, which is a contradiction, since $G_{F}$ is 2-connected. Therefore, $H_{i}$ is an $r_{i}-x_{i}$ chain. Similarly, we can show that $H_{i}$ is an $r_{i}-y_{i}$ chain.

(3.11) Notation. For each $H_{i} \in \mathcal{H}$ with $x_{i} \neq y_{i}$, let $A_{i}$ denote the block of $H_{i}$ containing $P_{i}$. If $A_{i} \neq H_{i}$, then let $b_{i}$ denote the cut vertex of $H_{i}$ contained in $A_{i}$. If $A_{i}=H_{i}$, then let $b_{i}:=r_{i}$.

The next lemma illustrates two situations when we can find a $B_{P}$-augmenting path.

(3.12) Lemma. Assume that $\left|X_{P}\right| \geq 3$ and let $H_{i} \in \mathcal{H}$. Suppose that one of the following holds:

(i) $x_{i}=y_{i}$, or

(ii) $x_{i} \neq y_{i}$, and $H_{i}$ contains at least three blocks or $H_{i}$ contains a nontrivial block other than $A_{i}$.

Then one can find a $B_{P}$-augmenting path in $O(|V(G)|+|E(G)|)$ time.

Proof. If $x_{i}=y_{i}$ then let $H:=H_{i}$. If $x_{i} \neq y_{i}$, then let $H:=H_{i}-\left(V\left(A_{i}\right)-\left\{b_{i}\right\}\right)$. Note that $H$ is an $r_{i}$ - $x_{i}$ chain if $x_{i}=y_{i}$, and $H$ is an $r_{i}$ - $b_{i}$ chain if $x_{i} \neq y_{i}$. Moreover, since (i) or (ii) holds, $H$ is not induced by an edge.

Let $H:=v_{0} B_{1} v_{1} \ldots v_{k-1} B_{k} v_{k}$ with $v_{0}=r_{i}, v_{k}=x_{i}$ if $x_{i}=y_{i}$, and $v_{k}=b_{i}$ if $x_{i} \neq y_{i}$. This decomposition of $H_{i}$ into blocks can be computed in $O(|V(G)|+|E(G)|)$ time.

Case 1: There exists $j \in\{1, \ldots, k\}$ such that $B_{j}$ is nontrivial.

Let $S:=N_{G}\left(B_{j}-\left\{v_{j-1}, v_{j}\right\}\right)-\left\{v_{j-1}, v_{j}\right\}$. Note that $S \subseteq V(F-r)-\left\{a, a^{\prime}\right\}$, because $B_{P}$ is a block of $G_{F}-V\left(P\left(a, a^{\prime}\right)\right)$. Let $G^{\prime}$ be the graph obtained from $B_{j}$ by adding $S$ and the edges of $G$ from $S$ to $V\left(B_{j}\right)-\left\{v_{j-1}, v_{j}\right\}$. Note that $G^{\prime}-S=B_{j}$ is 2connected and $G^{\prime}$ is $\left(4, S \cup\left\{v_{j-1}, v_{j}\right\}\right)$-connected (because $G$ is 4-connected). Therefore, the hypotheses of Lemma (2.3) are satisfied with $G^{\prime}, S, v_{j-1}, v_{j}$ as $G, S, b, b^{\prime}$, respectively. Then by Lemma (2.3) exactly one of the following occurs:

(1) there exist $u, u^{\prime} \in S$ and an induced $u-u^{\prime}$ path $P^{\prime}$ in $G^{\prime}$ such that $V\left(P^{\prime}\right) \cap\left\{v_{j-1}, v_{j}\right\}=$ $\emptyset, V\left(P^{\prime}\right) \cap S=\left\{u, u^{\prime}\right\}$, and $G^{\prime}-\left(V\left(P^{\prime}\right) \cup S\right)$ is connected, or

(2) $|S|=2$, and the elements of $S$ can be labeled as $u, u^{\prime}$ such that $\left(G^{\prime}, v_{j-1}, u, v_{j}, u^{\prime}\right)$ is planar. 
Moreover, one can in $O\left(V\left(G^{\prime}\right)|+| E\left(G^{\prime}\right) \mid\right.$ ) time (and hence, in $O(|V(G)|+|E(G)|$ ) time) find a path as in (1) or certify that (2) holds.

Note that since $\left|X_{P}\right| \geq 3$, there exists a path $W$ with ends in $X_{P}-\left\{r_{i}\right\}$ which is internally disjoint from $V\left(B_{P}\right) \cup V_{i}$.

Suppose (1) holds. We claim that $P^{\prime}$ is a feasible $F$-path. Clearly, $V\left(P^{\prime}\right) \cap V(F)=$ $\left\{u, u^{\prime}\right\}$ and $P^{\prime}$ is an induced path in $G-u u^{\prime}$. Since $B_{j}-V\left(P^{\prime}\left(u, u^{\prime}\right)\right)=G^{\prime}-\left(V\left(P^{\prime}\right) \cup S\right)$ is connected, and $v_{j-1}, v_{j} \notin V\left(P^{\prime}\right)$, we have that $G_{F}-V\left(P^{\prime}\left(u, u^{\prime}\right)\right)$ is connected. Thus, $P^{\prime}\left(u, u^{\prime}\right)$ is non-separating in $G_{F}$. Also $r \in V\left(B_{P}\right)$, and $B_{P} \subseteq G_{F}-V\left(P^{\prime}\left(u, u^{\prime}\right)\right)$. Therefore, since $r \notin\left\{u, u^{\prime}\right\}, P^{\prime}$ is a feasible $F$-path. Moreover, since $W$ is also a path in $G_{F}-V\left(P^{\prime}\left(u, u^{\prime}\right)\right), B_{P} \cup W \subseteq B_{P^{\prime}}$. Therefore, $P^{\prime}$ is a $B_{P^{-a u g m e n t i n g} \text { path. }}$

Now assume (2) holds. By Lemma (2.6) one can find in $O\left(\left|V\left(G^{\prime}\right)\right|+\left|E\left(G^{\prime}\right)\right|\right)$ time (and hence, in $O(|V(G)|+|E(G)|)$ time) an induced $u$ - $u^{\prime}$ path $Q$ in $G^{\prime}$ such that $G^{\prime}-V(Q)$ has exactly two components $K, K^{\prime}$ with $v_{j-1} \in V(K)$ and $v_{j} \in V\left(K^{\prime}\right)$. We claim that $Q$ is a feasible $F$-path. Clearly, $V(Q) \cap V(F)=\left\{u, u^{\prime}\right\}$, and $Q$ is an induced path in $G-u u^{\prime}$. Since $B-Q\left(u, u^{\prime}\right)=G^{\prime}-V(Q)$ has exactly two components (namely $K$ and $K^{\prime}$ ), there exists a path in $H_{i}$ from $v_{j-1} \in V(K)$ to $r_{i} \in X_{P}$ disjoint from $Q$, and there exists a path from $v_{j} \in V\left(K^{\prime}\right)$ to $X_{P}$ in $G_{F}-V\left(Q\left(u, u^{\prime}\right)\right)$ (because $\left|X_{P}\right| \geq 2$ ), it follows that $G_{F}-V\left(Q\left(u, u^{\prime}\right)\right)$ is connected. Also $r \in V\left(B_{P}\right)$, and $B_{P} \subseteq G_{F}-V\left(Q\left(u, u^{\prime}\right)\right)$. Since $r \notin\left\{u, u^{\prime}\right\}, Q$ is a feasible $F$-path. Moreover, $W$ is a path in $G_{F}-V\left(Q\left(u, u^{\prime}\right)\right)$, and hence, $B_{P} \cup W \subseteq B_{Q}$. Therefore, $Q$ is a $B_{P}$-augmenting path.

Case 2: All blocks of $H$ are trivial.

By (ii), $H_{i}$ contains at least two blocks other than $A_{i}$, and hence, $k \geq 3$. So $B_{1}$ and $B_{2}$ are trivial blocks of $H$. Since $G$ is 4-connected, $v_{1}$ has at least two neighbors in $V(F-r)$, say $u, u^{\prime}$. Let $P^{\prime}:=\left(u, v_{1}, u^{\prime}\right)$. We claim that $P^{\prime}$ is a feasible $F$-path. Clearly, $V\left(P^{\prime}\right) \cap V(F)=\left\{u, u^{\prime}\right\}$, and $P^{\prime}$ is an induced path in $G-u u^{\prime}$. Since $G_{F}$ is 2connected, $G_{F}-V\left(P^{\prime}\left(u, u^{\prime}\right)\right)=G_{F}-v_{1}$ is connected. Also since $B_{P} \subseteq G_{F}-V\left(P^{\prime}\left(u, u^{\prime}\right)\right)$, and $r \notin\left\{u, u^{\prime}\right\}$, it follows that $P^{\prime}$ is a feasible $F$-path. Moreover, one can see that $B_{P} \cup W \subseteq B_{P^{\prime}}$. Therefore, $P^{\prime}$ is a $B_{P^{-a u g m e n t i n g ~ p a t h . ~}}$

Now we study the case where, for every $H_{i} \in \mathcal{H}, x_{i} \neq y_{i}, H_{i}$ has at most two blocks, and if $H_{i}$ has exactly two blocks, then $A_{i}$ is the only nontrivial block of $H_{i}$. We give three lemmas which deal with this case. The arguments used for many cases in the proofs are similar, but unfortunately it seems necessary to cover all of those cases. We frequently produce a $B_{P}$-augmenting path $P^{\prime}$ in the following way. We first exhibit a nontrivial path $W$ in $G_{F}$ with ends in $B_{P}$ such that $W$ is internally disjoint from $B_{P}$. We then produce a feasible $F$-path $P^{\prime}$ disjoint from $W$ such that $V\left(B_{P}\right) \cup V(W) \subset V\left(B_{P^{\prime}}\right)$, so $P^{\prime}$ is $B_{P}$-augmenting. For the sake of brevity, when we state a result occurs "because of the path $W$," we are implicitly using this technique.

Recall that by (3.4) we may assume that if $|V(P)| \geq 4$, then $N_{G}\left(P\left(a, a^{\prime}\right)\right) \cap V(F) \subseteq$ $\left\{a, a^{\prime}\right\} \cup\{r\}$. 
(3.13) Lemma. Assume that $\left|X_{P}\right| \geq 3,|V(P)| \geq 4$, and, for every $H_{j} \in \mathcal{H}, x_{j} \neq y_{j}$. Suppose that, for every $H_{j} \in \mathcal{H}, V\left(A_{j}\right)-\left\{b_{j}, x_{j}, y_{j}\right\}$ has no neighbor in $V(F-r)-\left\{a, a^{\prime}\right\}$. Assume that for some $H_{i} \in \mathcal{H}, H_{i}$ is adjacent to $F$. Then exactly one of the following holds:

(1) there exists a $B_{P}$-augmenting path, or

(2) there exists a triangle $F$-chain $H$ in $G$ such that $I(H)=V\left(G_{F}\right)-V\left(B_{P}\right), G_{F}-I(H)$ is 2-connected, and $G[V(F) \cup I(H)]$ is 2-connected.

Moreover, one can in $O(|V(G)|+|E(G)|)$ time either find a path as in (1) or find a triangle $F$-chain as in (2).

Proof. Let us first show that (1) and (2) are mutually exclusive. Suppose that (2) holds. It is not hard to see that there exists no $B_{P}$-augmenting path because every feasible $F$-path must use exactly two vertices of $V\left(G_{F}\right)-V\left(B_{P}\right)$. Thus, it remains to show that either (1) or (2) holds, and one can determine in $O(|V(G)|+|E(G)|)$ time which of them occurs.

We consider two cases.

Case 1: There exist distinct $m, n \in\{1, \ldots, p\}-\{i\}$ such that both $V_{m}$ and $V_{n}$ have a neighbor in $V\left(P\left(x_{i}, a^{\prime}\right)\right)$, or both $V_{m}$ and $V_{n}$ have a neighbor in $V\left(P\left(a, y_{i}\right)\right)$.

Without loss of generality, assume that both $V_{m}$ and $V_{n}$ have a neighbor in $V\left(P\left(x_{i}, a^{\prime}\right)\right)$.

We claim that $A_{i}$ contains a non-separating induced $b_{i}-x_{i}$ path $Q$ such that $V(Q) \cap$ $\left(V\left(P_{i}\right)-\left\{x_{i}\right\}\right)=\emptyset$. This is obvious if $V\left(A_{i}\right)-V\left(P_{i}\right)=\left\{b_{i}\right\}$ because then $b_{i}$ must be adjacent to $x_{i}$, and the result follows by taking $Q$ as the path induced by the edge $b_{i} x_{i}$. Thus, we may assume that $V\left(A_{i}\right)-V\left(P_{i}\right) \neq\left\{b_{i}\right\}$. Let $S_{i}$ denote the set of vertices in $V\left(P\left(x_{i}, y_{i}\right)\right)$ which have a neighbor in $\left(\bigcup_{j=1}^{p} V_{j}\right)-V_{i}$. Since $G$ is 4-connected, $A_{i}$ is $\left(4, S_{i} \cup\left\{b_{i}, x_{i}, y_{i}\right\}\right)$-connected. Moreover, $A_{i}-\left(V\left(P_{i}\right)-\left\{x_{i}\right\}\right)$ is connected and $S_{i} \cup\left\{y_{i}\right\} \subseteq V\left(P_{i}\right)-\left\{x_{i}\right\}$, so there exists a $b_{i}-x_{i}$ path $Q^{\prime}$ in $A_{i}$ such that $V\left(P_{i}\right)-\left\{x_{i}\right\}$ (and hence, $S_{i} \cup\left\{y_{i}\right\}$ ) is contained in a component $U$ of $A_{i}-V\left(Q^{\prime}\right)$. Therefore, the hypotheses of (2.1) are satisfied with $A_{i}, S_{i} \cup\left\{b_{i}, x_{i}, y_{i}\right\}, b_{i}, x_{i}, Q^{\prime}, U$ as $G, S, a, a^{\prime}, P, U$, respectively. By (2.1) one can find in $O(|V(G)|+|E(G)|)$ time a non-separating induced $b_{i}-x_{i}$ path $Q$ in $A_{i}$ such that $V(Q) \cap V(U)=\emptyset$. Since $V\left(P_{i}\right)-\left\{x_{i}\right\} \subseteq V(U)$, we have $V(Q) \cap\left(V\left(P_{i}\right)-\left\{x_{i}\right\}\right)=\emptyset$, and thus, $Q$ is a path as required.

By hypothesis, $x_{i} \neq y_{i}$, so by (3.12), we can in $O(|V(G)|+|E(G)|)$ time either find a $B_{P}$-augmenting path, or certify that $H_{i}$ has at most two blocks. Hence, we may assume that $H_{i}$ has at most two blocks. Since $H_{i}$ is adjacent to $F$ and $V\left(A_{i}\right)-\left\{b_{i}, x_{i}, y_{i}\right\}$ has no neighbors in $V(F-r)-\left\{a, a^{\prime}\right\}$, it follows that $H_{i}$ has exactly two blocks, and $b_{i}$ is adjacent to some vertex $u \in V(F-r)-\left\{a, a^{\prime}\right\}$. Let $P^{\prime}:=\left(Q \cup P\left[a, x_{i}\right]\right)+\left\{u, b_{i} u\right\}$. By assumption, both $V_{m}$ and $V_{n}$ have a neighbor on $P\left(x_{i}, a^{\prime}\right)$. Since $P^{\prime}$ is disjoint from 
$V\left(P_{i}\right)-\left\{x_{i}\right\}$, there exists an $r_{m}-r_{n}$ path $W$ in $G_{F}-V\left(P^{\prime}(a, u)\right)$ which is internally disjoint from $V\left(B_{P}\right) \cup V_{i} \cup\left\{x_{i}\right\}$.

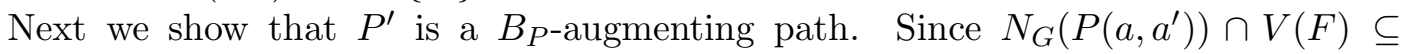
$\left\{a, a^{\prime}\right\} \cup\{r\}$ (by (3.4)), and $P$ is induced in $G-a a^{\prime}$, we have that $P^{\prime}$ is an induced $u$ - $a$ path in $G-a u$. Also, since $A_{i}-V(Q)$ is connected, $P^{\prime}(a, u)$ is non-separating in $G_{F}$. Note also that if $r$ is an end of $P^{\prime}$ then $a=r$, and $r$ is not a cut vertex of $G_{F}-V\left(P\left(a, a^{\prime}\right)\right)$. Then, because of the path $W, r$ is not a cut vertex of $G_{F}-V\left(P^{\prime}(a, u)\right)$. Thus, $P^{\prime}$ is a

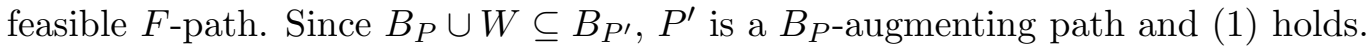

Case 2: For any distinct $m, n \in\{1, \ldots, p\}-\{i\}, V_{m}$ and $V_{n}$ do not both have a neighbor in $V\left(P\left(x_{i}, a^{\prime}\right)\right)$, nor do both $V_{m}$ and $V_{n}$ have a neighbor in $V\left(P\left(a, y_{i}\right)\right)$.

By hypothesis, $x_{i} \neq y_{i}$, so by (3.12), we can in $O(|V(G)|+|E(G)|)$ time either find a $B_{P}$-augmenting path or certify that $H_{i}$ has at most two blocks. Hence, we may assume that $H_{i}$ has at most two blocks. Since $A_{i}-\left\{b_{i}, x_{i}, y_{i}\right\}$ has no neighbor in $V(F-r)-$ $\left\{a, a^{\prime}\right\}$, it follows that $H_{i}$ has exactly two blocks, and $b_{i}$ has at least one neighbor in $V(F-r)-\left\{a, a^{\prime}\right\}$. Moreover, since we are in Case 2, we must have $\left|X_{P}\right|=3$. Without loss of generality, we may assume that $i=3, V_{1}$ has a neighbor in $V\left(P\left(a, x_{3}\right]\right)$, and $V_{2}$ has a neighbor in $V\left(P\left[y_{3}, a^{\prime}\right)\right)$. Moreover, $V_{1}$ has no neighbor in $V\left(P\left(x_{3}, a^{\prime}\right)\right)$, and $V_{2}$ has no neighbor in $V\left(P\left(a, y_{3}\right)\right)$.

Suppose $b_{3}$ has two neighbors in $V(F-r)-\left\{a, a^{\prime}\right\}$, say $u, u^{\prime}$. Let $P^{\prime}:=\left(u, b_{3}, u^{\prime}\right)$. Clearly, $G_{F}-V\left(P^{\prime}\left(u, u^{\prime}\right)\right)=G_{F}-b_{3}$ is connected. Since $r \notin\left\{u, u^{\prime}\right\}$, it is not hard to see that $P^{\prime}$ is a feasible $F$-path. Moreover, there exists an $r_{1}-r_{2}$ path which is internally disjoint from $V\left(B_{P}\right) \cup V_{i}$. Hence, $P^{\prime}$ is a $B_{P}$-augmenting path, and (1) holds. Clearly, $P^{\prime}$ can be found in $O(|V(G)|+|E(G)|)$ time.

Thus, we may assume that $b_{3}$ has exactly one neighbor in $V(F-r)-\left\{a, a^{\prime}\right\}$. We consider two subcases.

Subcase 2.1: For some $j \in\{1,2\}$, say $j=1, V_{1} \neq\{r\}$.

Let $H_{1}:=w_{0} B_{1}^{\prime} w_{1} \ldots w_{s-1} B_{s}^{\prime} w_{s}$ where $w_{0}=r_{1}$, and $B_{s}^{\prime}=A_{1}$. Since $x_{1} \neq y_{1}$ (by assumption), then from Lemma (3.12), either $s=1$, or $s=2$ and $B_{1}^{\prime}$ is trivial.

We claim that $V\left(A_{1}\right)=\left\{b_{1}, x_{1}, y_{1}\right\}$. Suppose for a contradiction that $V\left(A_{1}\right)$ $\left\{b_{1}, x_{1}, y_{1}\right\} \neq \emptyset$. Then $A_{1}-\left\{b_{1}, x_{1}, y_{1}\right\}$ is a component of $G-\left\{b_{1}, x_{1}, y_{1}\right\}$ for the following reasons: $V\left(A_{1}\right)-\left\{b_{1}, x_{1}, y_{1}\right\}$ has no neighbor in $V(F-r)-\left\{a, a^{\prime}\right\}$ (by hypothesis), $V\left(P\left(x_{1}, y_{1}\right)\right)$ has no neighbor in $V_{3} \cup V_{2}$ (by assumption in Case 2), and $P$ is an induced path in $G-a a^{\prime}$. But then $\left\{b_{1}, x_{1}, y_{1}\right\}$ is a 3 -cut in $G$ which contradicts the assumption that $G$ is 4 -connected. Thus, $V\left(A_{1}\right)=\left\{b_{1}, x_{1}, y_{1}\right\}$.

Therefore, $\left\{b_{1}, x_{1}, y_{1}\right\}$ induces a triangle in $G$. Since $H_{1} \in \mathcal{H}, V_{1} \neq\left\{r_{1}\right\}$. This implies that $s=2$ and $B_{1}^{\prime}$ is a trivial block of $H_{1}$ (and hence, $r_{1}$ is adjacent to $b_{1}$ ). Since $b_{1}$ has degree at least four in $G, b_{1}$ must have some neighbor in $V(F-r)$. Hence, $H_{1}$ is adjacent to $F$, and $V_{2}$ and $V_{3}$ have neighbors in $V\left(P\left(x_{1}, a^{\prime}\right)\right)$, so we can proceed as in Case 1 and find a $B_{P}$-augmenting path in $O(|V(G)|+|E(G)|)$ time. 
Subcase 2.2: For every $j \in\{1,2\}, V_{j}=\left\{r_{j}\right\}$.

Thus, $r_{1}$ has a neighbor in $V\left(P\left(a, x_{3}\right]\right)$, and hence, $x_{3} \neq a$. Similarly, $y_{3} \neq a^{\prime}$.

We claim that $V\left(A_{3}\right)=\left\{b_{3}, x_{3}, y_{3}\right\}$. Suppose for a contradiction that $V\left(A_{3}\right)-$ $\left\{b_{3}, x_{3}, y_{3}\right\} \neq \emptyset$. Then $A_{3}-\left\{b_{3}, x_{3}, y_{3}\right\}$ is a component of $G-\left\{b_{3}, x_{3}, y_{3}\right\}$ for the following reasons: $V\left(A_{3}\right)-\left\{b_{3}, x_{3}, y_{3}\right\}$ has no neighbor in $V(F-r)-\left\{a, a^{\prime}\right\}$ (by hypothesis), $V\left(P\left(x_{3}, y_{3}\right)\right)$ has no neighbor in $V_{1} \cup V_{2}$ (by assumption in Case 2), and $P$ is an induced path in $G-a a^{\prime}$. But then $\left\{b_{3}, x_{3}, y_{3}\right\}$ is a 3 -cut in $G$, which contradicts the assumption that $G$ is 4-connected. Thus, $V\left(A_{3}\right)=\left\{b_{3}, x_{3}, y_{3}\right\}$, and $A_{3}$ is a triangle.

Since $G_{F}$ is 2-connected and $P$ is an induced path in $G-a a^{\prime}$, and because $N_{G}\left(P\left(a, a^{\prime}\right)\right) \cap V(F) \subseteq\left\{a, a^{\prime}\right\} \cup\{r\}$, it follows that $V(P)=V\left(P_{3}\right) \cup\left\{a, a^{\prime}\right\}, r_{1}$ is adjacent to $x_{3}$, and $r_{2}$ is adjacent to $y_{3}$. Let $u$ denote the only neighbor of $b_{3}$ in $V(F-r)-\left\{a, a^{\prime}\right\}$. Note that $a \neq r$; otherwise $r_{1}=r$ (because $\left|X_{P}\right|=3$ ), and $x_{3}$ would have degree three in $G$ which is a contradiction, because $G$ is 4-connected. Similarly, $a^{\prime} \neq r$. If $r=r_{1}$, then $\left(r_{1}, x_{3}, a\right)$ is a $B_{P}$-augmenting path. If $r=r_{2}$, then $\left(r_{2}, y_{3}, a^{\prime}\right)$ is a $B_{P^{-a u g m e n t i n g}}$

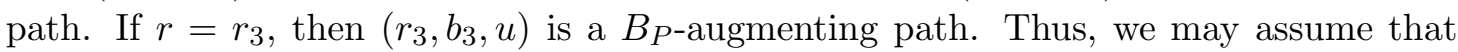
$r \notin\left\{r_{1}, r_{2}, r_{3}\right\}$. Therefore, $H:=A_{i}+\left\{u, a, a^{\prime}, b_{i} u, x_{i} a, y_{i} a^{\prime}\right\}$ is a triangle $F$-chain in $G$ with $b_{3}, x_{3}, y_{3}, u, a, a^{\prime}, r_{3}, r_{1}, r_{2}$ as $v_{1}, v_{2}, v_{3}, u_{1}, u_{2}, u_{3}, w_{1}, w_{2}, w_{3}$, respectively, in Definition (1.2). It is easy to see that $G_{F}-I(H)=B_{P}$ is 2-connected and $G[V(F) \cup I(H)]$ is 2-connected. So (2) holds.

(3.14) Lemma. Assume that $\left|X_{P}\right| \geq 3,|V(P)| \geq 4$, and for every $H_{j} \in \mathcal{H}, x_{j} \neq y_{j}$. Suppose that $H_{i} \in \mathcal{H}$ and $V\left(A_{i}\right)-\left\{b_{i}, x_{i}, y_{i}\right\}$ has a neighbor in $V(F-r)-\left\{a, a^{\prime}\right\}$. Assume that $V\left(P\left(x_{i}, y_{i}\right)\right)$ has no neighbor in $\left(\bigcup_{j=1}^{p} V_{j}\right)-V_{i}$. Then a $B_{P}$-augmenting path can be found in $O(|V(G)|+|E(G)|)$ time.

Proof. Since $G_{F}$ is 2-connected and $V\left(P\left(x_{i}, y_{i}\right)\right)$ has no neighbor in $\left(\bigcup_{j=1}^{p} V_{j}\right)-V_{i}$, there exists $m \in\{1, \ldots, p\}-\{i\}$ such that $V_{m}$ has a neighbor in $V\left(P\left(a, x_{i}\right]\right)$ or in $V\left(P\left[y_{i}, a^{\prime}\right)\right)$.

By symmetry we may assume that $V_{m}$ has a neighbor in $V\left(P\left[y_{i}, a^{\prime}\right)\right)$. Then $y_{i} \neq a^{\prime}$.

First, we find an endblock of $A_{i}-\left\{x_{i}, y_{i}\right\}$ in $O(|V(G)|+|E(G)|)$ time as follows. If $A_{i}-\left\{x_{i}, y_{i}\right\}$ is 2-connected, then let $B:=A_{i}-\left\{x_{i}, y_{i}\right\}$, and let $b:=b_{i}$. Otherwise, let $B$ be an endblock of $A_{i}-\left\{x_{i}, y_{i}\right\}$, and let $b$ denote the cut vertex of $A_{i}-\left\{x_{i}, y_{i}\right\}$ contained in $B$ so that $b_{i} \notin V(B-b)$. Note that since $V\left(P\left(x_{i}, y_{i}\right)\right)$ has no neighbors in $\left(\bigcup_{j=1}^{p} V_{j}\right)-V_{i}, N_{G}(B-b) \subseteq V(F-r) \cup\left\{x_{i}, y_{i}, b\right\}$. Since $r \notin\left\{x_{i}, y_{i}\right\}$ (by the definition of $x_{i}, y_{i}$ in (3.9)), $r \notin N_{G}(B-b)-\{b\}$. Moreover, since $G$ is 4-connected, $\left|N_{G}(B-b)\right| \geq 4$. Note that such an endblock $B$ can be found in $O(|V(G)|+|E(G)|)$ time.

Next, we consider two cases.

Case 1: $y_{i}$ has a neighbor in $V\left(A_{i}\right)-\left(\left\{x_{i}, y_{i}\right\} \cup V(B-b)\right)$.

Then, since $V_{m}$ has a neighbor in $V\left(P\left[y_{i}, a^{\prime}\right)\right)$, there exists an $r_{i}-r_{m}$ path $W$ in $G_{F}-V\left(P\left(a, x_{i}\right]\right)$ which is internally disjoint from $V\left(B_{P}\right)$ and intersects $P\left[y_{i}, a^{\prime}\right)$. 
Subcase 1.1: $B$ is trivial.

Let $v$ denote the unique vertex in $V(B)-\{b\}$. Then $N_{G}(v) \subseteq V(F-r) \cup\left\{x_{i}, y_{i}, b\right\}$. Since $G$ is 4-connected, $v$ has at least three neighbors in $V(F-r) \cup\left\{x_{i}, y_{i}\right\}$, and hence, it has two neighbors in $V(F-r) \cup\left\{x_{i}\right\}$. Let $u, u^{\prime}$ be distinct neighbors of $v$ in $V(F-$ $r) \cup\left\{x_{i}\right\}$, and assume that $u \neq x_{i}$. By the definition of $x_{i}, y_{i}$ in (3.9), one can see that $\left\{u, u^{\prime}\right\} \cap\left\{a^{\prime}\right\}=\emptyset$ and $u \neq a$ (because $y_{i} \neq a^{\prime}$ and $x_{i} \neq u$ ). If $u^{\prime} \neq x_{i}$, then let $P^{\prime}:=\left(u, v, u^{\prime}\right)$; otherwise, let $P^{\prime}:=P\left[a, x_{i}\right]+\left\{u, v, u v, v x_{i}\right\}$. Clearly, $P^{\prime}$ is a path with ends in $V(F)$ and internally disjoint from $V\left(B_{P}\right) \cup V(F)$.

Next we show that $P^{\prime}$ is a $B_{P}$-augmenting path. Let $u, u^{\prime \prime}$ denote the ends of $P^{\prime}$. By assumption, $N_{G}\left(P\left(a, a^{\prime}\right)\right) \cap V(F) \subseteq\left\{a, a^{\prime}\right\} \cup\{r\}$ (by (3.4)), and $P$ is induced in $G-a a^{\prime}$. Then since $N_{G}(v) \subseteq V(F-r) \cup\left\{x_{i}, y_{i}, b\right\}$, and $V\left(P\left[a, x_{i}\right)\right)$ has no neighbor in $V(B)$ (by the definition of $x_{i}$ in (3.9)), it follows that $P^{\prime}$ is induced in $G-u u^{\prime \prime}$. Because of the path $W$, and since $P\left(a, a^{\prime}\right)$ is non-separating in $G_{F}, G_{F}-V\left(P^{\prime}\left(u, u^{\prime}\right)\right.$ is connected. So $P^{\prime}\left(u, u^{\prime \prime}\right)$ is non-separating in $G_{F}$. If $r \in\left\{u, u^{\prime \prime}\right\}$, then since $r \notin\left\{u, u^{\prime}\right\}, r=u^{\prime \prime}=a$ and $r$ is not a cut vertex of $G_{F}-V\left(P\left(a, a^{\prime}\right)\right)$. Then, because of the path $W, r$ is not a cut vertex of $G_{F}-V\left(P^{\prime}\left(u, u^{\prime \prime}\right)\right)$. Thus, $P^{\prime}$ is a feasible $F$-path. Since $B_{P} \cup W \subseteq B_{P^{\prime}}, P^{\prime}$ is a $B_{P}$-augmenting path. Clearly, $P^{\prime}$ can be found in $O(|V(G)|+|E(G)|)$ time.

Subcase 1.2: $B$ is nontrivial.

Let $S:=N_{G}(B-b)-\left\{b, y_{i}\right\}$ and let $G^{\prime}$ be obtained from $B$ by adding $S$ and the edges of $G$ from $S$ to $V(B)-\{b\}$; since $r \notin N_{G}(B-b), r \notin S$. Since $G$ is 4connected, $|S| \geq 2$ and $G^{\prime}$ is $\left(3, S \cup\{b\}\right.$ )-connected (if $y_{i} \notin N_{G}(B-b)$ then actually

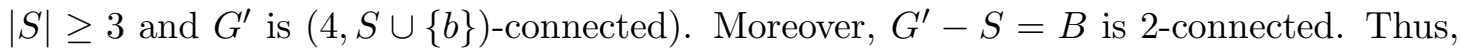
the hypotheses of Lemma (2.4) are satisfied with $G^{\prime}, S, b$ as $G, S, b$, respectively. Then there exist $u, u^{\prime} \in S$ and an induced $u-u^{\prime}$ path $Q$ in $G^{\prime}$ such that $V(Q) \cap\{b\}=\emptyset$, $V(Q) \cap S=\left\{u, u^{\prime}\right\}$, and $G^{\prime}-(V(Q) \cup S)$ is connected. Moreover, such a path $Q$ can be found in $O\left(\left|V\left(G^{\prime}\right)\right|+\left|E\left(G^{\prime}\right)\right|\right)$ time (and hence, in $O(|V(G)|+|E(G)|)$ time).

By the definition of $x_{i}, y_{i}$ in (3.9) and because $y_{i} \neq a^{\prime},\left\{u, u^{\prime}\right\} \cap\left\{a^{\prime}\right\}=\emptyset$. By symmetry we may assume that $u \neq x_{i}$. If $u^{\prime} \neq x_{i}$, then let $P^{\prime}:=Q$; otherwise let $P^{\prime}:=P\left[a, x_{i}\right] \cup Q$. Clearly, $P^{\prime}$ is a path with ends in $V(F)$ and internally disjoint from $V\left(B_{P}\right) \cup V(F)$.

Next we show that $P^{\prime}$ is a $B_{P}$-augmenting path. Let $u, u^{\prime \prime}$ denote the ends of $P^{\prime}$. By assumption, $N_{G}\left(P\left(a, a^{\prime}\right)\right) \cap V(F) \subseteq\left\{a, a^{\prime}\right\} \cup\{r\}$ (by (3.4)) and $P$ is induced in $G-a a^{\prime}$. Then since $Q$ is induced in $G^{\prime}$ and $P\left[a, x_{i}\right)$ has no neighbor in $V(B)$ (by the definition of $x_{i}$ in (3.9)), it follows that $P^{\prime}$ is induced in $G-u u^{\prime \prime}$. Since $B-V\left(Q\left(u, u^{\prime}\right)\right)=G^{\prime}-(V(Q) \cup S)$ is connected and because of the path $W, P^{\prime}\left(u, u^{\prime \prime}\right)$ is non-separating in $G_{F}$. If $r \in\left\{u, u^{\prime \prime}\right\}$, then since $r \notin S, r=u^{\prime \prime}=a$, and $r$ is not a cut vertex of $G_{F}-V\left(P\left(a, a^{\prime}\right)\right)$. Then, because of the path $W, r$ is not a cut vertex of $G_{F}-V\left(P^{\prime}\left(u, u^{\prime \prime}\right)\right)$. Thus, $P^{\prime}$ is a feasible

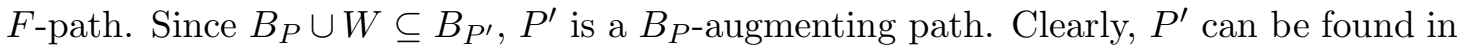
$O(|V(G)|+|E(G)|)$ time.

Case 2: $y_{i}$ has no neighbor in $V\left(A_{i}\right)-\left(\left\{x_{i}, y_{i}\right\} \cup V(B-b)\right)$ (and hence, $y_{i} \in N_{G}(B-b)$ ). 
Subcase 2.1: $B$ is trivial.

Let $v$ denote the unique vertex in $V(B)-\{b\}$. Then $N_{G}(v) \subseteq V(F-r) \cup\left\{x_{i}, y_{i}, b\right\}$, and $y_{i}$ is adjacent to $v$. Since $G$ is 4-connected, $v$ has at least four neighbors in $V(F-$ $r) \cup\left\{x_{i}, y_{i}, b\right\}$, and hence, it has at least two neighbors in $V(F-r) \cup\left\{x_{i}\right\}$. Let $u, u^{\prime} \in$ $N_{G}(v)-\left\{b, y_{i}\right\}$, and assume that $u \neq x_{i}$. By the definition of $x_{i}, y_{i}$ in (3.9), one can see that $\left\{u, u^{\prime}\right\} \cap\left\{a^{\prime}\right\}=\emptyset$ (because $y_{i} \neq a^{\prime}$ ) and $u \neq a$ (because $u \neq x_{i}$ ).

Suppose that there exists $n \in\{1, \ldots, p\}-\{i, m\}$ such that $V_{n}$ has a neighbor in $V\left(P\left[y_{i}, a^{\prime}\right)\right)$. Then there exists an $r_{m}-r_{n}$ path $W$ in $G_{F}-V\left(P\left(a, y_{i}\right)\right)$ which is internally disjoint from $V\left(B_{P}\right)$ and intersects $P\left[y_{i}, a^{\prime}\right)$. If $u^{\prime} \neq x_{i}$, then let $P^{\prime}:=\left(u, v, u^{\prime}\right)$; otherwise let $P^{\prime}:=P\left[a, x_{i}\right]+\left\{u, v, u v, v x_{i}\right\}$. Then $P^{\prime}$ is a path with ends in $V(F)$ and internally disjoint from $V\left(B_{P}\right) \cup V(F)$. Next we show that $P^{\prime}$ is a $B_{P}$-augmenting path. Let $u, u^{\prime \prime}$ denote the ends of $P^{\prime}$. By assumption, $N_{G}\left(P\left(a, a^{\prime}\right)\right) \cap V(F) \subseteq\left\{a, a^{\prime}\right\} \cup\{r\}$ (by (3.4)) and $P$ is induced in $G-a a^{\prime}$. Then, since $N_{G}(v) \subseteq V(F-r) \cup\left\{x_{i}, y_{i}, b\right\}$ and $V\left(P\left[a, x_{i}\right)\right)$ has no neighbor in $V(B)$ (by the definition of $x_{i}$ in (3.9)), it follows that $P^{\prime}$ is an induced path in $G-u u^{\prime \prime}$. Because of the path $W$ and since $P\left(a, a^{\prime}\right)$ is non-separating in $G_{F}$, $G_{F}-V\left(P^{\prime}\left(u, u^{\prime \prime}\right)\right)$ is connected, and so $P^{\prime}\left(u, u^{\prime \prime}\right)$ is non-separating in $G_{F}$. If $r \in\left\{u, u^{\prime \prime}\right\}$, then since $r \notin\left\{u, u^{\prime}\right\}, r=u^{\prime \prime}=a$, and $r$ is not a cut vertex of $G_{F}-V\left(P\left(a, a^{\prime}\right)\right)$. Then, because of the path $W, r$ is not a cut vertex of $G_{F}-V\left(P^{\prime}\left(u, u^{\prime \prime}\right)\right)$. Thus, $P^{\prime}$ is a feasible $F$-path. Since $B_{P} \cup W \subseteq B_{P^{\prime}}, P^{\prime}$ is a $B_{P^{-a u g m e n t i n g ~ p a t h . ~}}$

Thus, we may assume that there exists no $n \in\{1, \ldots, p\}-\{i, m\}$ such that $V_{n}$ has a neighbor in $V\left(P\left[y_{i}, a^{\prime}\right)\right)$. Since $\left|X_{P}\right| \geq 3$ and $V\left(P\left(x_{i}, y_{i}\right)\right)$ has no neighbor in $\left(\bigcup_{j=1}^{p} V_{j}\right)-V_{i}$, we have that $x_{i} \neq a$, and there exists $n \in\{1, \ldots, p\}-\{i, m\}$ such that $V_{n}$ has a neighbor in $V\left(P\left(a, x_{i}\right]\right)$. Furthermore, $x_{i}$ has a neighbor in $V\left(A_{i}\right)-\left\{x_{i}, y_{i}, v\right\}$; otherwise, since $y_{i}$ has no neighbor in $V\left(A_{i}\right)-\left(\left\{x_{i}, y_{i}\right\} \cup V(B-b)\right), v$ would be a cut vertex of $A_{i}$. Therefore, there exists an $r_{i}-r_{n}$ path $W$ in $G_{F}-V\left(P^{\prime}\left[y_{i}, a^{\prime}\right)\right)$ which is internally disjoint from $V\left(B_{P}\right)$ and intersects $P\left(a, x_{i}\right]$.

Let $P^{\prime}:=P\left[y_{i}, a^{\prime}\right]+\left\{u, v, u v, v y_{i}\right\}$. Then $P^{\prime}$ is a path with ends in $V(F)$ and internally disjoint from $V\left(B_{P}\right) \cup V(F)$. One can show that $P^{\prime}$ is an induced path in $G-u a^{\prime}$, and because of the path $W, P^{\prime}\left(u, a^{\prime}\right)$ is non-separating in $G_{F}$. Since $u \neq x_{i} \neq r, r$ is an end of $P^{\prime}$ only if $a^{\prime}=r$. In this case, $r$ is not a cut vertex of $G_{F}-V\left(P\left(a, a^{\prime}\right)\right)$, and because of the path $W, r$ is not a cut vertex of $G_{F}-V\left(P^{\prime}\left(u, a^{\prime}\right)\right)$. Thus, $P^{\prime}$ is a feasible $F$-path. Since $B_{P} \cup W \subseteq B_{P^{\prime}}, P^{\prime}$ is a $B_{P}$-augmenting path. Note that in all above cases, such a path $P^{\prime}$ can be found in $O(|V(G)|+|E(G)|)$ time.

Subcase 2.2: $B$ is nontrivial.

First, we define a graph $G^{\prime}$ from $B$. If $y_{i}$ has at least two neighbors in $V(B)$ then let $S:=N_{G}(B-b)-\left\{b, y_{i}\right\}$, let $G^{\prime}$ be obtained from $B$ by adding $S \cup\left\{y_{i}\right\}$ and the edges of $G$ from $S \cup\left\{y_{i}\right\}$ to $V(B)-\{b\}$, and let $y^{*}:=y_{i}$. If $y_{i}$ has exactly one neighbor in $V(B)$, then let $y^{*}$ denote this vertex (note that $y^{*} \neq b$ because $y_{i} \in N_{G}(B-b)$ by assumption), let $S:=N_{G}\left(B-\left\{b, y^{*}\right\}\right)$, and let $G^{\prime}$ be obtained from $B$ by adding $S$ and 
the edges of $G$ from $S$ to $V(B)-\left\{b, y^{*}\right\}$. Note that in either case $S \subseteq V(F-r) \cup\left\{x_{i}\right\}$. Moreover, $G^{\prime}-S=B$ is 2-connected, and $G^{\prime}$ is $\left(4, S \cup\left\{b, y^{*}\right\}\right.$ )-connected (because $G$ is 4 -connected). Thus, the hypotheses in (2.3) are satisfied with $G^{\prime}, S, b, y^{*}$ as $G, S, b, b^{\prime}$, respectively. By (2.3) exactly one of the following holds:

(1) there exist $u, u^{\prime} \in S$ and an induced $u$ - $u^{\prime}$ path $Q$ in $G^{\prime}$ such that $V(Q) \cap\left\{b, y^{*}\right\}=\emptyset$, $V(Q) \cap S=\left\{u, u^{\prime}\right\}$, and $G^{\prime}-(V(Q) \cup S)$ is connected; or

(2) $|S|=2$, and the elements of $S$ can be labeled as $u, u^{\prime}$ such that $\left(G^{\prime}, u, b, u^{\prime}, y^{*}\right)$ is planar.

Moreover, one can in $O\left(\left|V\left(G^{\prime}\right)\right|+\left|E\left(G^{\prime}\right)\right|\right)$ time (and hence, in $O(|V(G)|+|E(G)|)$ time) find a path as in (1) or certify that (2) holds. Without loss of generality, we may assume that $u \neq x_{i}$.

Suppose (1) occurs. If $u^{\prime} \neq x_{i}$ then let $P^{\prime}:=Q$; otherwise let $P^{\prime}:=P\left[a, x_{i}\right] \cup Q$. Then $P^{\prime}$ is a path with ends in $V(F)$ and internally disjoint from $V\left(B_{P}\right) \cup V(F)$. Since $y^{*}$ and $b$ are in $G^{\prime}-(V(Q) \cup S)$ which is connected, and because $V_{m}$ has a neighbor in $V\left(P\left[y_{i}, a^{\prime}\right)\right)$, there exists an $r_{i}-r_{m}$ path $W$ in $G_{F}-V\left(P\left(a, y_{i}\right)\right)$ which is internally disjoint from $V\left(B_{P}\right) \cup V(F)$ and intersects $P\left[y_{i}, a^{\prime}\right)$.

Next we show that $P^{\prime}$ is a $B_{P}$-augmenting path. Let $u, u^{\prime \prime}$ denote the ends of $P^{\prime}$. Since $Q$ is induced in $G^{\prime}$ and $N_{G}\left(P\left(a, a^{\prime}\right)\right) \cap V(F) \subseteq\left\{a, a^{\prime}\right\} \cup\{r\}$, and because $P$ is induced in $G-a a^{\prime}$ and $P\left[a, x_{i}\right)$ has no neighbor in $V(B)$ (by the definition of $x_{i}$ in (3.9)), one can see that $P^{\prime}$ is an induced path in $G-u u^{\prime \prime}$. Because of the path $W$, and since $P\left(a, a^{\prime}\right)$ is non-separating in $G_{F}, P^{\prime}\left(u, u^{\prime \prime}\right)$ is non-separating in $G_{F}$. Since $r \notin S$, if $r \in\left\{u, u^{\prime \prime}\right\}$, then $r=u^{\prime \prime}=a$, and $r$ is not a cut vertex of $G_{F}-V\left(P\left(a, a^{\prime}\right)\right)$. Then, because of the path $W, r$ is not a cut vertex of $G_{F}-V\left(P^{\prime}\left(u, u^{\prime \prime}\right)\right)$. Thus, $P^{\prime}$ is a feasible $F$-path. Since $B_{P} \cup W \subseteq B_{P^{\prime}}, P^{\prime}$ is a $B_{P}$-augmenting path.

So we may assume (2) occurs. We consider two cases.

First, assume there exists $n \in\{1, \ldots, p\}-\{i, m\}$ such that $V_{n}$ has a neighbor in $V\left(P\left[y_{i}, a^{\prime}\right)\right)$. Then there exists an $r_{m}-r_{n}$ path $W$ in $G_{F}-V\left(P\left(a, y_{i}\right)\right)$ which is internally disjoint from $V\left(B_{P}\right) \cup V(F)$ and intersects $P\left[y_{i}, a^{\prime}\right)$. By Lemma (2.6) (with $G^{\prime}, u, u^{\prime}, b, y^{*}$ as $G, a, a^{\prime}, b, b^{\prime}$, respectively), there exists an induced $u-u^{\prime}$ path $Q$ in $G^{\prime}$ such that $G^{\prime}-$ $V(Q)$ has exactly two components $K$ and $K^{\prime}$ with $b \in V(K)$ and $y^{*} \in V\left(K^{\prime}\right)$. Moreover, such a path can be found in $O\left(\left|V\left(G^{\prime}\right)\right|+\left|E\left(G^{\prime}\right)\right|\right.$ ) time (and hence, in $O(|V(G)|+E(G) \mid$ ) time). If $u^{\prime} \neq x_{i}$, then let $P^{\prime}:=Q$; otherwise let $P^{\prime}:=P\left[a, x_{i}\right] \cup Q$. So $P^{\prime}$ is a path with ends in $V(F)$ which is internally disjoint from $V\left(B_{P}\right) \cup V(F)$.

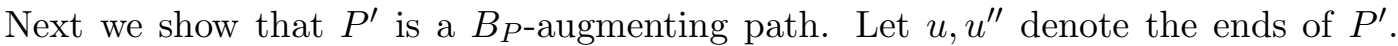
Since $Q$ is induced in $G^{\prime}$ and $N_{G}\left(P\left(a, a^{\prime}\right)\right) \cap V(F) \subseteq\left\{a, a^{\prime}\right\} \cup\{r\}$ (by (3.4)), and because $P$ is induced in $G-a a^{\prime}$ and $P\left[a, x_{i}\right)$ has no neighbor in $V(B)$ (by the definition of $x_{i}$ in $(3.9))$, one can see that $P^{\prime}$ is an induced path in $G-u u^{\prime \prime}$. Since $G^{\prime}-V(Q)$ has exactly two components, one containing $b$ and the other containing $y^{*}$, and because of the path 
$W$, it follows that $P^{\prime}\left(u, u^{\prime \prime}\right)$ is non-separating in $G_{F}$. If $r \in\left\{u, u^{\prime \prime}\right\}$, then $r=u^{\prime \prime}=a$, and $r$ is not a cut vertex of $G_{F}-V\left(P\left(a, a^{\prime}\right)\right)$. Then, because of the path $W, r$ is not a cut vertex of $G-V\left(P^{\prime}\left(u, u^{\prime \prime}\right)\right)$. Thus, $P^{\prime}$ is a feasible $F$-path. Since $B_{P} \cup W \subseteq B_{P^{\prime}}, P^{\prime}$ is a $B_{P}$-augmenting path.

Now assume that there exists no $n \in\{1, \ldots, p\}-\{i, m\}$ such that $V_{n}$ has a neighbor in $V\left(P\left[y_{i}, a^{\prime}\right)\right)$. Since $\left|X_{P}\right| \geq 3$ and $V\left(P\left(x_{i}, y_{i}\right)\right)$ has no neighbor in $\left(\bigcup_{j=1}^{p} V_{j}\right)-V_{i}$, there exists $n \in\{1, \ldots, p\}-\{i, m\}$ such that $V_{n}$ has a neighbor in $V\left(P\left(a, x_{i}\right]\right)$, and hence, $x_{i} \neq a$. Note that $G^{\prime} \neq K_{1,4}$ because $B$ is nontrivial. By Lemma (2.7) (with $G^{\prime}, u, y^{*}, u^{\prime}, b$ as $G, a, a^{\prime}, b, b^{\prime}$, respectively), there exists a non-separating induced $u-y^{*}$ path $Q$ in $G^{\prime}$ such that $V(Q) \cap\left\{u^{\prime}, b\right\}=\emptyset$. Moreover, such a path can be found in $O\left(\left|V\left(G^{\prime}\right)\right|+\left|E\left(G^{\prime}\right)\right|\right)$ time (and hence, in $O(|V(G)|+|E(G)|)$ time). Note that either $x_{i}$ has a neighbor in $V\left(A_{i}\right)-V(B-b)$ or $x_{i}$ is in $G^{\prime}-V(Q)$. Since $V_{n}$ has a neighbor in $V\left(P\left(a, x_{i}\right]\right)$, there exists an $r_{i}-r_{n}$ path $W$ in $G_{F}-V\left(P\left(x_{i}, a^{\prime}\right)\right)$ which is internally disjoint from $V\left(B_{P}\right) \cup V(F)$ and intersects $P\left(a, x_{i}\right]$. If $y^{*}=y_{i}$, then let $P^{\prime}:=Q \cup P\left[y_{i}, a^{\prime}\right]$; otherwise let $P^{\prime}:=\left(Q \cup P\left[y_{i}, a^{\prime}\right]\right)+\left\{y_{i}, y_{i} y^{*}\right\}$. One can show that $P^{\prime}$ is an induced path in $G-u a^{\prime}$, and because of the path $W, P^{\prime}\left(u, a^{\prime}\right)$ is non-separating in $G_{F}$. If $r \in\left\{u, a^{\prime}\right\}$, then $a^{\prime}=r$, and $r$ is not a cut vertex of $G_{F}-V\left(P^{\prime}\left(u, a^{\prime}\right)\right)$, because of the path $W$ and $r$ is not a cut vertex of $G_{F}-V\left(P\left(a, a^{\prime}\right)\right)$. Thus, $P^{\prime}$ is a feasible $F$-path. Since $B_{P} \cup W \subseteq B_{P^{\prime}}$, $P^{\prime}$ is a $B_{P}$-augmenting path.

(3.15) Lemma. Assume that $\left|X_{P}\right| \geq 3,|V(P)| \geq 4$, and, for every $H_{j} \in \mathcal{H}, x_{j} \neq y_{j}$. Suppose that $H_{i} \in \mathcal{H}$ and $V\left(A_{i}\right)-\left\{b_{i}, x_{i}, y_{i}\right\}$ has a neighbor in $V(F-r)-\left\{a, a^{\prime}\right\}$. Assume that $V\left(P\left(x_{i}, y_{i}\right)\right)$ has a neighbor in $\left(\bigcup_{j=1}^{p} V_{j}\right)-V_{i}$. Then a $B_{P}$-augmenting path can be found in $O(|V(G)|+|E(G)|)$ time.

Proof. Since $\left|X_{P}\right| \geq 3$ and $V\left(P\left(x_{i}, y_{i}\right)\right)$ has a neighbor in $\left(\bigcup_{j=1}^{p} V_{j}\right)-V_{i}$, there exist $m, n \in\{1, \ldots, p\}-\{i\}$ such that both $V_{m}$ and $V_{n}$ have a neighbor in $V\left(P\left(a, y_{i}\right)\right)$, or both $V_{m}$ and $V_{n}$ have a neighbor in $V\left(P\left(x_{i}, a^{\prime}\right)\right)$.

By symmetry we may assume that both $V_{m}$ and $V_{n}$ have a neighbor in $V\left(P\left(x_{i}, a^{\prime}\right)\right)$. Therefore, there exists an $r_{m}-r_{n}$ path $W$ in $G_{F}-V\left(P\left(a, x_{i}\right]\right)$ which is internally disjoint from $V\left(B_{P}\right) \cup V(F)$ and intersects $P\left(x_{i}, a^{\prime}\right)$.

Let $D$ be the graph obtained from $A_{i}-\left\{x_{i}, y_{i}\right\}$ by adding a new vertex $b^{\prime}$ and new edges from $b^{\prime}$ to each $v \in V\left(P\left(x_{i}, y_{i}\right)\right)$ such that $v$ has a neighbor in some $V_{j}$, $j \in\{1, \ldots, p\}-\{i\}$. Since $P\left(x_{i}, y_{i}\right) \subseteq A_{i}-\left\{x_{i}, y_{i}\right\}, N_{D}\left(b^{\prime}\right) \cup\left\{b^{\prime}\right\}$ is contained in a block of $D$, and $b^{\prime}$ is not a cut vertex of $D$. Note also that if $D$ is not connected, then $D$ has exactly two components, one containing $b_{i}$ and the other induced by $V\left(P\left(x_{i}, y_{i}\right)\right) \cup\left\{b^{\prime}\right\}$, and the component containing $b^{\prime}$ is a block of $D$ since every vertex in $V\left(P\left(x_{i}, y_{i}\right)\right)$ has a neighbor in some $V_{j}, j \neq i$ (because $N_{G}\left(P\left(a, a^{\prime}\right)\right) \cap V(F) \subseteq\left\{a, a^{\prime}\right\} \cup\{r\}$ by (3.4)). We consider two cases.

Case 1: $D$ is not a $b_{i}-b^{\prime}$ chain. 
Then there exists an endblock $B$ of $D$ such that one of the following holds: (1) $b^{\prime} \notin$ $V(B)$, and if $b_{i} \in V(B)$, then $b_{i}$ is a cut vertex of $D$, or (2) $D$ has exactly two components and $B$ is the component of $D$ containing $b_{i}$ (and hence, $V(B) \cap\left(V\left(P\left(x_{i}, y_{i}\right)\right) \cup\left\{b^{\prime}\right\}\right)=\emptyset$ by the argument in the last paragraph). Note that such an endblock can be found in $O(|V(G)|+|E(G)|)$ time. If (1) holds then let $b$ denote the cut vertex of $D$ contained in $B$. If (2) holds, then let $b:=b_{i}$. Since $\left|X_{P}\right| \geq 3$ and $B_{P}$ is a block of $G_{F}-V\left(P\left(a, a^{\prime}\right)\right)$, it follows from the definition of $x_{i}, y_{i}$ in (3.9) that $r \notin\left\{x_{i}, y_{i}\right\}$. Note that $N_{D}\left(b^{\prime}\right) \cap V(B-b)=$ $\emptyset$ and $r \notin N_{G}(B-b)$.

Subcase 1.1: $B$ is trivial.

Let $v$ denote the only vertex in $V(B)-\{b\}$. Note that $N_{G}(v) \subseteq V(F-r) \cup\left\{x_{i}, y_{i}, b\right\}$. Since $G$ is 4-connected and $N_{D}\left(b^{\prime}\right) \cap V(B-b)=\emptyset, v$ has at least three neighbors in $V(F-r) \cup\left\{x_{i}, y_{i}\right\}$. Let $u, u^{\prime}$ be two distinct neighbors of $v$ in $V(F-r) \cup\left\{x_{i}\right\}$. By symmetry, we may assume that $u \neq x_{i}$. If $u^{\prime} \neq x_{i}$, then let $P^{\prime}:=\left(u, v, u^{\prime}\right)$. If $u^{\prime}=x_{i}$, then let $P^{\prime}:=P\left[a, x_{i}\right]+\left\{u, v, u v, v x_{i}\right\}$. Then $P^{\prime}$ is path with ends in $V(F)$ which is internally disjoint from $V\left(B_{P}\right) \cup V(F)$.

Next we show that $P^{\prime}$ is a $B_{P}$-augmenting path. Let $u, u^{\prime \prime}$ denote the ends of $P^{\prime}$. By assumption, $N_{G}\left(P\left(a, a^{\prime}\right)\right) \cap V(F) \subseteq\left\{a, a^{\prime}\right\} \cup\{r\}$, and $P$ is induced in $G-a a^{\prime}$. Since $N_{G}(v) \subseteq V(F-r) \cup\left\{x_{i}, y_{i}, b\right\}$, and $V\left(P\left[a, x_{i}\right)\right)$ has no neighbor in $V(B)$ (by the definition of $x_{i}$ in (3.9)), it follows that $P^{\prime}$ is induced in $G-u u^{\prime \prime}$. Because of the path $W$ and since $P\left(a, a^{\prime}\right)$ is non-separating in $G_{F}, P^{\prime}\left(u, u^{\prime \prime}\right)$ is non-separating in $G_{F}$. Moreover, if $r \in\left\{u, u^{\prime \prime}\right\}$ then $r=u^{\prime \prime}=a$, and $r$ is not a cut vertex of $G_{F}-V\left(P\left(a, a^{\prime}\right)\right)$. Then, because of the path $W, r$ is not a cut vertex of $G_{F}-V\left(P^{\prime}(u, a)\right)$. Thus, $P^{\prime}$ is a feasible $F$-path. Since $B_{P} \cup W \subseteq B_{P^{\prime}}, P^{\prime}$ is a $B_{P^{-a u g m e n t i n g ~ p a t h . ~}}$

Subcase 1.2: $B$ is nontrivial.

Let $S:=N_{G}(B-b)-\left\{y_{i}, b\right\}$, and let $G^{\prime}$ be obtained from $B$ by adding $S$ and the edges of $G$ from $S$ to $V(B)-\{b\}$. Note that since $r \notin\left\{x_{i}, y_{i}\right\}$ and $r \notin N_{G}(B-b), r \notin S$. Since $G$ is 4-connected and $y_{i}$ is the only possible neighbor of $V(B-b)$ not in $S \cup\{b\}$, $G^{\prime}$ is $(3, S \cup\{b\})$-connected. By Lemma (2.4) (with $G^{\prime}, S, b$ as $G, S, b$, respectively) there exist $u, u^{\prime} \in S$ and an induced $u$ - $u^{\prime}$ path $Q$ in $G^{\prime}$ such that $V(Q) \cap\{b\}=\emptyset$, $V(Q) \cap S=\left\{u, u^{\prime}\right\}$, and $G^{\prime}-(V(Q) \cup S)$ is connected. Moreover, such a path can be found in $O\left(\left|V\left(G^{\prime}\right)\right|+\left|E\left(G^{\prime}\right)\right|\right)$ time (and hence, in $O(|V(G)|+|E(G)|)$ time). Without loss of generality, we may assume that $u \neq x_{i}$. If $u^{\prime} \neq x_{i}$, then let $P^{\prime}:=Q$; otherwise let $P^{\prime}:=P\left[a, x_{i}\right] \cup Q$. Then $P^{\prime}$ is a path with ends in $V(F)$ which is internally disjoint from $V\left(B_{P}\right) \cup V(F)$.

Next we prove that $P^{\prime}$ is a $B_{P}$-augmenting path. Let $u, u^{\prime \prime}$ denote the ends of $P^{\prime}$. Note that $Q$ is induced in $G-u u^{\prime \prime}, N_{G}\left(P\left(a, a^{\prime}\right)\right) \cap V(F) \subseteq\left\{a, a^{\prime}\right\} \cup\{r\}$ (by (3.4)), and $P$ is induced in $G-a a^{\prime}$. Then since $N_{G}(v) \subseteq V(F-r) \cup\left\{x_{i}, y_{i}, b\right\}$, and $V\left(P\left[a, x_{i}\right)\right)$ has no neighbor in $V(B)$ (by the definition of $x_{i}$ in (3.9)), it follows that $P^{\prime}$ is induced in $G-u u^{\prime \prime}$. Because of the path $W$ and since $G^{\prime}-(V(Q) \cup S)$ is connected, $P^{\prime}\left(u, u^{\prime \prime}\right)$ is non-separating 
in $G_{F}$. If $r \in\left\{u, u^{\prime \prime}\right\}$, then $r=u^{\prime \prime}=a$, and $r$ is not a cut vertex of $G_{F}-V\left(P\left(a, a^{\prime}\right)\right)$. Then, because of the path $W, r$ is not a cut vertex of $G_{F}-V\left(P^{\prime}\left(u, u^{\prime \prime}\right)\right)$. Thus, $P^{\prime}$ is a

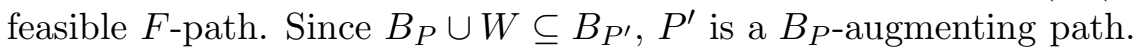

Case 2: $D$ is a $b_{i}-b^{\prime}$ chain.

Let $D:=w_{0} B_{1} w_{1} \ldots w_{l-1} B_{l} w_{l}$ where $w_{0}:=b_{i}$ and $w_{l}=b^{\prime}$. Note that this block decomposition can be found in $O(|V(G)|+|E(G)|)$ time.

For each nontrivial block $B_{j}$ with $1 \leq j \leq l-1$, let $S_{j}:=N_{G}\left(B_{j}-\left\{w_{j-1}, w_{j}\right\}\right)$. If $B_{l}$ is nontrivial, then let $S_{l}:=N_{G}\left(B_{l}-\left\{w_{l-1}, w_{l}\right\}\right)-X_{P}$, namely, $S_{l}$ contains all neighbors of $V\left(B_{l}-\left\{w_{l-1}, w_{l}\right\}\right)$ except the neighbors of $N_{D}\left(b^{\prime}\right)$ contained in $X_{P}$. For each nontrivial block $B_{j}$ with $1 \leq j \leq l$, let $G_{j}$ be obtained from $B_{j}$ by adding $S_{j}$ and the edges of $G$ from $S_{j}$ to $V\left(B_{j}\right)$. Note that $N_{D}\left(b^{\prime}\right) \cup\left\{b^{\prime}\right\} \subseteq B_{l}$, and for $1 \leq j \leq l-1$, $V\left(B_{j}\right) \cap\left(N_{D}\left(b^{\prime}\right) \cup\left\{b^{\prime}\right\}\right)=\emptyset$. Hence, for $1 \leq j \leq l-1, S_{j} \subseteq V(F-r) \cup\left\{x_{i}, y_{i}\right\}$. Moreover, $r \notin\left\{x_{i}, y_{i}\right\}$ by (3.9). Thus, $r \notin S_{j}$ for $1 \leq j \leq l-1$. Also if $B_{l}$ is nontrivial, then no vertex in $V\left(B_{l}-N_{D}\left(b^{\prime}\right)\right)$ is adjacent to $r$, and by the definition of $S_{l}, r \notin S_{l}$. First, we prove the following.

Claim. One can in $O(|V(G)|+|E(G)|)$ time either find a $B_{P}$-augmenting path or certify that the following statements hold:

(I) for each nontrivial block $B_{j}$ with $1 \leq j \leq l-1,\left|S_{j}\right|=2, y_{i} \in S_{j}$, and if $u$ denotes the vertex in $S_{j}-\left\{y_{i}\right\}$, then $\left(G_{j}, y_{i}, w_{j-1}, u, w_{j}\right)$ is planar,

(II) for each $1 \leq j \leq l-2$ for which both $B_{j}, B_{j+1}$ are trivial, $\left|N_{G}\left(w_{j}\right)-\left\{w_{j-1}, w_{j+1}\right\}\right|=$ 2 and $y_{i} \in N_{G}\left(w_{j}\right)$, and

(III) if $B_{l}$ is nontrivial, then $S_{l} \cap\left(V(F-r)-\left\{a, a^{\prime}\right\}\right)=\emptyset$.

Proof of Claim. We will show that if one of (I)-(III) does not hold, then one can find in $O(|V(G)|+|E(G)|)$ time a $B_{P}$-augmenting path.

Proof of (I). Suppose that $j \in\{1, \ldots, l-1\}$ and $B_{j}$ is nontrivial. Note that $G_{j}-S_{j}=B_{j}$ is 2-connected. Moreover, since $G$ is 4-connected, $G_{j}$ is $\left(4, S_{j} \cup\left\{w_{j-1}, w_{j}\right\}\right)$-connected. Thus, the hypotheses of (2.3) are satisfied with $G_{j}, S_{j}, w_{j-1}, w_{j}$ as $G, S, b, b^{\prime}$ respectively. By (2.3) exactly one of the following holds:

(1) there exist $u, u^{\prime} \in S_{j}$ and a $u-u^{\prime}$ path $Q$ such that $V(Q) \cap\left\{w_{j-1}, w_{j}\right\}=\emptyset, V(Q) \cap S_{j}=$ $\left\{u, u^{\prime}\right\}$, and $G_{j}-\left(V(Q) \cup S_{j}\right)$ is connected, or

(2) $\left|S_{j}\right|=2$, and the elements of $S_{j}$ can be labeled as $u, u^{\prime}$ such that $\left(G_{j}, u, w_{j-1}, u^{\prime}, w_{j}\right)$ is planar. 
Moreover, one can in $O\left(\left|V\left(G_{j}\right)\right|+\left|E\left(G_{j}\right)\right|\right)$ time (and hence, in $O(|V(G)|+|E(G)|)$ time) find a path as in (1) or certify that (2) holds.

Suppose that (1) holds. Define $P^{\prime}$ as follows.

(a) if $\left\{u, u^{\prime}\right\} \cap\left\{x_{i}, y_{i}\right\}=\emptyset$, then let $P^{\prime}:=Q$,

(b) if $\left\{u, u^{\prime}\right\}=\left\{x_{i}, y_{i}\right\}$, then let $P^{\prime}:=\left(P-V\left(P\left(x_{i}, y_{i}\right)\right)\right) \cup Q$,

(c) if $\left\{u, u^{\prime}\right\} \cap\left\{x_{i}, y_{i}\right\}=\left\{x_{i}\right\}$, then let $P^{\prime}:=P\left[a, x_{i}\right] \cup Q$, and

(d) if $\left\{u, u^{\prime}\right\} \cap\left\{x_{i}, y_{i}\right\}=\left\{y_{i}\right\}$, then let $P^{\prime}:=P\left[y_{i}, a^{\prime}\right] \cup Q$.

We claim that $P^{\prime}$ is a path with ends in $V(F)$ which is internally disjoint from $V\left(B_{P}\right) \cup$ $V(F)$. If (a) or (b) occurs, then clearly $P^{\prime}$ is a path as claimed. Suppose (c) occurs, that is, $\left\{u, u^{\prime}\right\} \cap\left\{x_{i}, y_{i}\right\}=\left\{x_{i}\right\}$. If $a \notin\left\{u, u^{\prime}\right\}$, then clearly $P^{\prime}$ is a path as claimed; if $a \in\left\{u, u^{\prime}\right\}$, then by the definition of $x_{i}$ in (3.9), $x_{i}=a$, and hence, $P^{\prime}$ is a path as claimed. Similarly, if (d) occurs, then $P^{\prime}$ is a path as claimed.

Next we show that $P^{\prime}$ is a $B_{P^{-a u g m e n t i n g}}$ path. Let $u_{1}, u_{2}$ denote the ends of $P^{\prime}$. Since $Q$ is induced in $G_{j}$ and $N_{G}\left(P\left(a, a^{\prime}\right)\right) \cap V(F) \subseteq\{a, a\} \cup\{r\}$ (by (3.4)), and because $P$ is induced in $G-a a^{\prime}$ and $P\left[a, x_{i}\right)$ has no neighbor in $V\left(B_{j}\right)$ (by the definition of $x_{i}$ in $(3.9)$ ), one can show that $P^{\prime}$ is an induced path in $G-u_{1} u_{2}$. Since $G_{j}-\left(V(Q) \cup S_{j}\right)$ is connected, it is easy to see that $P^{\prime}\left(u_{1}, u_{2}\right)$ is non-separating in $G_{F}$. If $r \in\left\{u_{1}, u_{2}\right\}$, then since $r \notin\left\{u, u^{\prime}\right\} \subseteq S_{j}$, (b), (c) or (d) occurs, and either $r=a$ or $r=a^{\prime}$. In this case, $r$ is not a cut vertex of $G_{F}-V\left(P\left(a, a^{\prime}\right)\right)$, and since $\left|X_{P}\right| \geq 3, r$ is not a cut vertex of $G_{F}-V\left(P^{\prime}\left(u_{1}, u_{2}\right)\right)$. Thus, $P^{\prime}$ is a feasible $F$-path. Since there exists a $w_{j-1}-w_{j}$ path in $G_{j}-\left(V(Q) \cup S_{j}\right)$, there exists an $r_{i}-b^{\prime}$ path in $D-V\left(P^{\prime}\left(u_{1}, u_{2}\right)\right)$. By the definition of $b^{\prime}$, the vertex adjacent to $b^{\prime}$ in this path has a neighbor in $V_{t}$ for some $t \in\{1, \ldots, p\}-\{i\}$. Hence, $B_{P}$ is properly contained in a block of $G_{F}-V\left(P^{\prime}\left(u_{1}, u_{2}\right)\right)$, and therefore, $P^{\prime}$ is a $B_{P}$-augmenting path.

So assume that (2) holds. If $y_{i} \in\left\{u, u^{\prime}\right\}$ then (I) holds, so we may assume that $y_{i} \notin\left\{u, u^{\prime}\right\}$. By Lemma (2.6) with $G_{j}, u, u^{\prime}, w_{j-1}, w_{j}$ as $G, a, a^{\prime}, b, b^{\prime}$, respectively, one can find in $O\left(\left|V\left(G_{j}\right)\right|+\left|E\left(G_{j}\right)\right|\right)$ time an induced $u$ - $u^{\prime}$ path $Q$ such that $G_{j}-V(Q)$ has exactly two components $K$ and $K^{\prime}$ with $w_{j-1} \in V(K)$ and $w_{j} \in V(K)$. Without loss of generality, we may assume that $u \neq x_{i}$. If $u^{\prime} \neq x_{i}$, then let $P^{\prime}:=Q$; otherwise let $P^{\prime}:=P\left[a, x_{i}\right] \cup Q$. Clearly, $P^{\prime}$ is a path with ends in $V(F)$ which is internally disjoint from $V\left(B_{P}\right) \cup V(F)$.

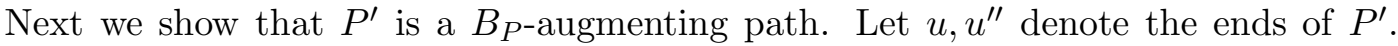
Since $Q$ is induced in $G_{j}$ and $N_{G}\left(P\left(a, a^{\prime}\right)\right) \cap V(F) \subseteq\left\{a, a^{\prime}\right\} \cup\{r\}$, and because $P$ is an induced path in $G-a a^{\prime}$ and $P\left[a, x_{i}\right)$ has no neighbor in $V\left(B_{j}\right)$ (by the definition of $x_{i}$ in (3.9)), one can show that $P^{\prime}$ is an induced path in $G-u u^{\prime \prime}$. Since $G_{j}-V(Q)$ has exactly two components, one containing $w_{j-1}$ and the other containing $w_{j}$, and because of the path $W$, it follows that $G-V\left(P^{\prime}\left(u, u^{\prime \prime}\right)\right)$ is connected, so $P^{\prime}$ is non-separating in 
$G_{F}$. If $r \in\left\{u, u^{\prime \prime}\right\}$, then $r=u^{\prime \prime}=a$, and $r$ is not a cut vertex of $G_{F}-V\left(P\left(a, a^{\prime}\right)\right)$. Then, because of the path $W, r$ is not a cut vertex of $G_{F}-V\left(P^{\prime}\left(u, u^{\prime \prime}\right)\right.$. Thus, $P^{\prime}$ is a feasible $F$-path. Since $B_{P} \cup W \subseteq B_{P^{\prime}}, P^{\prime}$ is a $B_{P^{-a u g m e n t i n g ~ p a t h . ~}}$

Proof of (II). Suppose that for some $j \in\{1, \ldots, l-1\}$ both $B_{j}$ and $B_{j+1}$ are trivial. If $y_{i} \in N_{G}\left(w_{j}\right)$ and $\left|N_{G}\left(w_{j}\right)-\left\{w_{j-1}, w_{j}\right\}\right|=2$ then (II) holds, so we may assume that $y_{i} \notin N_{G}\left(w_{j}\right)$ or $\left|N_{G}\left(w_{j}\right)-\left\{w_{j-1}, w_{j}\right\}\right| \neq 2$. Therefore, $\left|N_{G}\left(w_{j}\right)-\left\{w_{j-1}, w_{j}, y_{i}\right\}\right| \geq 2$. Let $u, u^{\prime}$ be distinct vertices in $N_{G}\left(w_{j}\right)-\left\{w_{j-1}, w_{j}, y_{i}\right\}$. Note that $r \notin\left\{u, u^{\prime}\right\}$ because $B_{P}$ is a block of $G_{F}-V\left(P\left(a, a^{\prime}\right)\right)$. Without loss of generality we may assume that $u \neq x_{i}$. If $u^{\prime} \neq x_{i}$ then let $P^{\prime}:=\left(u, w_{j}, u^{\prime}\right)$. If $u^{\prime}=x_{i}$ then let $P^{\prime}:=P\left[a, x_{i}\right]+\left\{u, w_{j}, w_{j} x_{i}, u w_{j}\right\}$. By the definition of $x_{i}, y_{i}$ in (3.9), $u \neq a$. So $P^{\prime}$ is a path with ends in $V(F)$ which is internally disjoint from $V\left(B_{P}\right) \cup V(F)$. Note that $V\left(P^{\prime}\right) \cap V\left(P\left(x_{i}, a^{\prime}\right)\right)=\emptyset$.

Next we show that $P^{\prime}$ is a $B_{P}$-augmenting path. Let $u, u^{\prime \prime}$ denote the ends of $P$. Since $P$ is an induced path in $G-a a^{\prime}$, and because $w_{j}$ has no neighbor in $P\left[a, x_{i}\right.$ ) (by the definition of $x_{i}$ in (3.9)), one can see that $P^{\prime}$ is induced in $G-u u^{\prime \prime}$. Because of the path $W$ and since $P\left(a, a^{\prime}\right)$ is non-separating in $G_{F}, P^{\prime}$ is non-separating in $G_{F}$. If $r \in\left\{u, u^{\prime \prime}\right\}$, then $r=u^{\prime \prime}=a$, and $r$ is not a cut vertex of $G_{F}-V\left(P\left(a, a^{\prime}\right)\right)$. Then, because of the path $W, r$ is not a cut vertex of $G_{F}-V\left(P^{\prime}\left(u, u^{\prime \prime}\right)\right.$. Thus, $P^{\prime}$ is a feasible $F$-path. Since $B_{P} \cup W \subseteq B_{P^{\prime}}, P^{\prime}$ is a $B_{P^{-a u g m e n t i n g ~ p a t h . ~}}$

Proof of (III). Suppose $B_{l}$ is nontrivial. If $S_{l} \cap\left(V(F-r)-\left\{a, a^{\prime}\right\}\right)=\emptyset$ then (III) holds, so we may assume that $S_{l} \cap\left(V(F-r)-\left\{a, a^{\prime}\right\}\right) \neq \emptyset$. We want to apply (2.3) to find a $B_{P^{-}}$ augmenting path, so we need to show that $G_{l}, S_{l}, w_{l-1}, w_{l}=b^{\prime}$ (as $G, S, b, b^{\prime}$, respectively) satisfy the hypotheses in the statement of (2.3). Clearly, $G_{l}-S_{l}=B_{l}$ is 2-connected and by definition, every vertex in $S_{l}$ has a neighbor in $V\left(B_{l}\right)-\left\{w_{l-1}, w_{l}\right\}$. Since $P$ is an induced path in $G-a a^{\prime}$ and $G$ is 4-connected, $G_{l}-b^{\prime} \subseteq G$ is $\left(4, S_{j} \cup\left\{w_{l-1}\right\} \cup N_{D}\left(b^{\prime}\right)\right)$ connected. Hence, $G_{l}$ is $\left(3, S_{l} \cup\left\{w_{l-1}, b^{\prime}\right\}\right)$-connected. Recall that $r \notin S_{l}$ (see the definition of $\left.S_{l}\right), V\left(P\left(x_{i}, y_{i}\right)\right)$ has no neighbor in $V(F-r)-\left\{a, a^{\prime}\right\}$ unless $x_{i}=a$ or $y_{i}=a^{\prime}$ (by (3.4)), and $A_{i}-V\left(P_{i}\right)$ is connected. Thus, since $S_{l} \cap\left(V(F-r)-\left\{a, a^{\prime}\right\}\right) \neq \emptyset$, $V\left(B_{l}\right)-\left(\left\{w_{l-1}, w_{l}\right\} \cup V\left(P\left(x_{i}, y_{i}\right)\right)\right) \neq \emptyset$. This implies that $V\left(P\left(x_{i}, y_{i}\right)\right) \subset V\left(B_{l}\right)-$ $\left\{w_{l-1}, w_{l}\right\}$ (and hence, $\left.x_{i}, y_{i} \in S_{l}\right)$, for otherwise $w_{l-1} \in V\left(P\left(x_{i}, y_{i}\right)\right)$, contradicting the fact that $A_{i}-V\left(P_{i}\right)$ is connected. Thus, $\left|S_{l}\right| \geq 3$, and there exists a component $K$ of $G_{l}-\left(S_{l} \cup\left\{w_{l-1}, w_{l}\right\}\right)=B_{l}-\left\{w_{l-1}, w_{l}\right\}$ which contains $V\left(P\left(x_{i}, y_{i}\right)\right)$. Note that $K$ has at least two neighbors in $S_{l}$, namely, $x_{i}, y_{i}$. Thus, the hypotheses of (2.3) are satisfied with $G_{l}, S_{l}, w_{l-1}, w_{l}$ as $G, S, b, b^{\prime}$, respectively.

Therefore, by (2.3) there exist $u, u^{\prime} \in S_{l}$ and an induced path $Q$ in $G_{l}$ such that $V(Q) \cap\left\{w_{l-1}, w_{l}\right\}=\emptyset, V(Q) \cap S_{l}=\left\{u, u^{\prime}\right\}$, and $G_{l}-\left(V(Q) \cup S_{l}\right)$ is connected. Define $P^{\prime}$ as follows.

(a) if $\left\{u, u^{\prime}\right\} \cap\left\{x_{i}, y_{i}\right\}=\emptyset$, then let $P^{\prime}:=Q$,

(b) if $\left\{u, u^{\prime}\right\}=\left\{x_{i}, y_{i}\right\}$, then let $P^{\prime}:=\left(P-V\left(P\left(x_{i}, y_{i}\right)\right)\right) \cup Q$, 
(c) if $\left\{u, u^{\prime}\right\} \cap\left\{x_{i}, y_{i}\right\}=\left\{x_{i}\right\}$, then let $P^{\prime}:=P\left[a, x_{i}\right] \cup Q$, and

(d) if $\left\{u, u^{\prime}\right\} \cap\left\{x_{i}, y_{i}\right\}=\left\{y_{i}\right\}$, then let $P^{\prime}:=P\left[y_{i}, a^{\prime}\right] \cup Q$.

We claim that $P^{\prime}$ is a path with ends in $V(F)$ which is internally disjoint from $V\left(B_{P}\right) \cup$ $V(F)$. Clearly, this is true if (a) or (b) occurs. Suppose (c) occurs, that is, $\left\{u, u^{\prime}\right\} \cap$ $\left\{x_{i}, y_{i}\right\}=\left\{x_{i}\right\}$. If $a \notin\left\{u, u^{\prime}\right\}$, then $P^{\prime}$ is a path as claimed. If $a \in\left\{u, u^{\prime}\right\}$, then by the definition of $x_{i}$ in (3.9), $a=x_{i}$. Again, $P^{\prime}$ is a path as claimed. Similarly, if (d) occurs, then $P^{\prime}$ is a path as claimed.

Next we show that $P^{\prime}$ is a $B_{P}$-augmenting path. Let $u_{1}, u_{2}$ denote the ends of $P^{\prime}$. Since $Q$ is induced in $G_{l}$ and $N_{G}\left(P\left(a, a^{\prime}\right)\right) \cap V(F) \subseteq\{a, a\} \cup\{r\}$, and because $P$ is induced in $G-a a^{\prime}$ and $P\left[a, x_{i}\right)$ has no neighbor in $B_{l}$ (by the definition of $x_{i}$ in (3.9)), one can see that $P^{\prime}$ is an induced path in $G-u_{1} u_{2}$. Since $G_{l}-\left(V(Q) \cup S_{l}\right)$ is connected, and $V\left(P\left(x_{i}, y_{i}\right)\right)$ has a neighbor in $\left(\bigcup_{j=1}^{p} V_{j}\right)-V_{i}$, it is easy to see that $P^{\prime}\left(u_{1}, u_{2}\right)$ is non-separating in $G_{F}$. If $r \in\left\{u_{1}, u_{2}\right\}$, then since $r \notin S_{l}$, (b), (c) or (d) occurs, and either $r=a$ or $r=a^{\prime}$. In this case, $r$ is not a cut vertex of $G_{F}-V\left(P\left(a, a^{\prime}\right)\right)$, and since $\left|X_{P}\right| \geq 3, r$ is not a cut vertex of $G_{F}-V\left(P^{\prime}\left(u_{1}, u_{2}\right)\right)$. Thus, $P^{\prime}$ is a feasible $F$-path. Moreover, since there exists a $w_{l-1}-w_{l}$ path $W^{\prime}$ in $G_{l}-\left(V(Q) \cup S_{l}\right)$, there exists an $r_{i}-b^{\prime}$ path $W^{\prime \prime}$ in $D-V\left(P^{\prime}\left(u_{1}, u_{2}\right)\right)$. By the definition of $b^{\prime}$, the vertex adjacent to $b^{\prime}$ in $W^{\prime \prime}$ has a neighbor in $V_{t}$ for some $t \in\{1, \ldots, p\}-\{i\}$. Hence, $B_{P}$ is properly contained in a

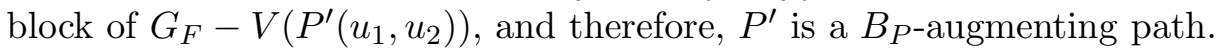

This concludes the proof of the claim.

By the above claim, we may assume that (I), (II) and (III) hold. Therefore, by (III) and since $V\left(A_{i}\right)-\left\{b_{i}, x_{i}, y_{i}\right\}$ has a neighbor in $V(F-r)-\left\{a, a^{\prime}\right\}$, we have $l \geq 2$. We consider three subcases.

Subcase 2.1: $x_{i}$ has at least two neighbors in $V\left(B_{l}\right)$.

Thus, $B_{l}$ is nontrivial (because $x_{i}$ is not adjacent to $b^{\prime}$ in $D$ ). We claim that $P\left(x_{i}, y_{i}\right) \subseteq B_{l}-w_{l-1}$. Suppose for a contradiction that $P\left(x_{i}, y_{i}\right) \nsubseteq B_{l}-w_{l-1}$. Then $w_{l-1} \in V\left(P\left(x_{i}, y_{i}\right)\right)$. Since $G_{F}-V\left(P\left(a, a^{\prime}\right)\right)$ is connected, $B_{l}-b^{\prime} \subseteq P\left(x_{i}, y_{i}\right)$. But then $x_{i}$ has at most one neighbor in $V\left(B_{l}\right)$ because $P$ is an induced path in $G_{F}-a a^{\prime}$, a contradiction. Therefore, $P\left(x_{i}, y_{i}\right) \subseteq B_{l}-w_{l-1}$.

Since $V\left(A_{i}\right)-\left\{b_{i}, x_{i}, y_{i}\right\}$ has a neighbor in $V(F-r)-\left\{a, a^{\prime}\right\}$ and $S_{l} \cap(V(F-r)-$ $\left.\left\{a, a^{\prime}\right\}\right)=\emptyset$ by (III), there exists $q \in\{1, \ldots, l-1\}$ such that $V\left(B_{q}-w_{q-1}\right)$ has a neighbor in $V(F-r)-\left\{a, a^{\prime}\right\}$. Choose $q$ to be maximum with this property and let $u$ be a neighbor of $V\left(B_{q}-w_{q-1}\right)$ in $V(F-r)-\left\{a, a^{\prime}\right\}$.

Next we define a $u-w_{q}$ path $Q_{q}$ in $G_{q}$. If $B_{q}$ is trivial or $u$ is adjacent to $w_{q}$, then let $Q_{q}$ be the path induced by the edge $u w_{q}$. Otherwise, $B_{q}$ is nontrivial, $S_{q}=\left\{u, y_{i}\right\}$, and $\left(G_{q}, y_{i}, w_{q-1}, u, w_{q}\right)$ is planar (by (I)). By Lemma (2.7) (with $G_{q}, u, w_{q}, y_{i}, w_{q-1}$ as $G, a, a^{\prime}, b, b^{\prime}$, respectively), there exists a non-separating induced $u-w_{q}$ path $Q_{q}$ in $G_{q}$ such 
that $V\left(Q_{q}\right) \cap\left\{y_{i}, w_{q-1}\right\}=\emptyset$. Moreover, such a path can be found in $O\left(\left|V\left(G_{q}\right)\right|+\left|E\left(G_{q}\right)\right|\right)$ time.

By the maximality of $q$, for $q+1 \leq j \leq l-1$, the following holds: if $B_{j}$ is nontrivial, then $S_{j}=\left\{x_{i}, y_{i}\right\}$ and $\left(G_{j}, y_{i}, w_{j-1}, x_{i}, w_{j}\right)$ is planar (by (I)), and if $B_{j}$ and $B_{j+1}$ are trivial, then $N_{G}\left(w_{j}\right)-\left\{w_{j-1}, w_{j+1}\right\}=\left\{x_{i}, y_{i}\right\}$ (by (II)). Note also that $x_{i} \in S_{l}$ because $P\left(x_{i}, y_{i}\right) \subseteq B_{l}-w_{l-1}$.

Choose the minimum $t \in\{q+1, \ldots, l\}$ such that $x_{i} \in N_{G}\left(B_{t}-w_{t}\right)$. Thus, by the choice of $q$ and $t, B_{j}$ is trivial for every $j \in\{q+1, \ldots, t-1\}$. For each $j \in\{q+1, \ldots, t-1\}$, let $Z_{j}$ denote the path induced by the edge $w_{j-1} w_{j}$.

If $B_{t}$ is trivial, then let $Q_{t}$ denote the path induced by the edge $w_{t-1} x_{i}$. If $B_{t}$ is nontrivial, then we define a path $Q_{t}$ according to the following two cases.

- $t<l$. Then $S_{t}=\left\{x_{i}, y_{i}\right\}$, and $\left(G_{t}, w_{t-1}, x_{i}, w_{t}, y_{i}\right)$ is planar. By Lemma (2.7) with $G_{t}, w_{t-1}, x_{i}, w_{t}, y_{i}$ as $G, a, a^{\prime}, b, b^{\prime}$, respectively, there exists a non-separating induced $w_{t-1}-x_{i}$ path $Q_{t}$ in $G_{t}$ such that $V\left(Q_{t}\right) \cap\left\{w_{t}, y_{i}\right\}=\emptyset$. Moreover, such a path can be found in $O\left(\left|V\left(G_{t}\right)\right|+\left|E\left(G_{t}\right)\right|\right)$ time.

- $t=l$. Since $P$ is induced in $G-a a^{\prime}$ and $x_{i}$ has at least two neighbors in $V\left(B_{l}\right), x_{i}$ has a neighbor in $V\left(B_{l}\right)-V\left(P\left(x_{i}, y_{i}\right)\right)$. Moreover, $B_{l}-V\left(P\left(x_{i}, y_{i}\right)\right)$ is connected, because $A_{i}-V\left(P_{i}\right)$ is connected, and hence, there exists a $w_{l-1}-x_{i}$ path $Q^{\prime}$ in $B_{l}-V\left(P\left(x_{i}, y_{i}\right)\right)$. Let $G^{\prime}:=G_{l}-b^{\prime}$ and let $S^{\prime}:=N_{D}\left(b^{\prime}\right) \cup S_{l} \cup\left\{w_{l-1}\right\}$. Then $G^{\prime}$ is $\left(4, S^{\prime}\right)$-connected, and $S^{\prime}-\left\{w_{l-1}, x_{i}\right\}$ is contained in a component $U$ of $G^{\prime}-V\left(Q^{\prime}\right)$. By Lemma (2.1) (with $G^{\prime}, S^{\prime}, w_{l-1}, x_{i}, U$ as $G, S, a, a^{\prime}, U$, respectively) there exists a non-separating induced $w_{l-1}-x_{i}$ path $Q_{l}$ in $G^{\prime}$ such that $V\left(Q_{l}\right) \cap V(U)=\emptyset$ (and hence, $\left.V\left(Q_{l}\right) \cap V\left(P\left(x_{i}, y_{i}\right)\right)=\emptyset\right)$. Moreover, such a path can be found in $O\left(\left|V\left(G^{\prime}\right)\right|+\left|E\left(G^{\prime}\right)\right|\right)$ time (and hence, in $O(|V(G)|+|E(G)|)$ time).

Let $P^{\prime}:=Q_{q} \cup Z_{q+1} \cup \ldots \cup Z_{t-1} \cup Q_{t} \cup P\left[a, x_{i}\right]$. Then $P^{\prime}$ is a $u-a$ path in $G$ such that $V\left(P^{\prime}\right) \cap V(F)=\{u, a\}$. Moreover, it is not hard to see that such a path can be found in $O(|V(G)|+|E(G)|)$ time.

Next we show that $P^{\prime}$ is a $B_{P}$-augmenting path. It is not hard to see that $P^{\prime}$ is an induced path in $G-u a$. Because of the path $W$ and since $P\left(a, a^{\prime}\right)$ is non-separating in $G_{F}$, $P^{\prime}(u, a)$ is non-separating in $G_{F}$. If $a=r$, then $r$ is not a cut vertex of $G_{F}-V\left(P\left(a, a^{\prime}\right)\right)$, and because of the path $W, r$ is not a cut vertex of $G_{F}-V\left(P^{\prime}(u, a)\right)$. Thus, $P^{\prime}$ is a feasible $F$-path. Moreover, since $V\left(P^{\prime}\right) \cap V\left(P\left(x_{i}, a^{\prime}\right]\right)=\emptyset, B_{P} \cup W \subseteq B_{P^{\prime}}$. Therefore, $P^{\prime}$ is a $B_{P}$-augmenting path.

Subcase 2.2: $x_{i}$ has at most one neighbor in $V\left(B_{l}\right)$, and $x_{i}$ has a neighbor in $V\left(A_{i}\right)-$ $\left(V\left(P\left(x_{i}, y_{i}\right)\right) \cup\left\{b_{i}\right\}\right)$.

Then since $A_{i}$ is 2-connected, $x_{i}$ has a neighbor in $V(D)-\left(V\left(B_{l}\right) \cup\left\{b_{i}\right\}\right)$. Therefore, since $V\left(A_{i}\right)-\left\{b_{i}, x_{i}, y_{i}\right\}$ has a neighbor in $V(F-r)-\left\{a, a^{\prime}\right\}$ and by (I), (II) and (III), 
there exist $u \in V(F-r)-\left\{a, a^{\prime}\right\}$ and $q, t \in\{1, \ldots, l-1\}$ with $q \leq t$ such that one of the following holds:

(a) $u \in N_{G}\left(B_{q}-w_{q-1}\right)$ and $x_{i} \in N_{G}\left(B_{t}-w_{t}\right)$, or

(b) $x_{i} \in N_{G}\left(B_{q}-w_{q-1}\right)$, and $u \in N_{G}\left(B_{t}-w_{t}\right)$.

Choose $q, t$ so that $t-q$ is minimum, and (a) or (b) holds. Note that $q<t$ because in (I) we must have $y_{j} \in S_{j}$, and in (II) we must have $y_{j} \in N_{G}\left(w_{j}\right)$.

We may assume that (a) holds, because the other case is symmetric.

By the minimality of $t-q$ and by (I), $B_{j}$ is trivial for every $j \in\{q+1, \ldots, t-1\}$. Using (II), one can also show that $t-q \leq 2$. For $q+1 \leq j \leq t-1$, let $Z_{j}$ denote the path induced by the edge $w_{j-1} w_{j}$.

If $B_{q}$ is trivial, then let $Q_{q}$ be the path induced by the edge $u w_{q}$. Otherwise (by (I)) $B_{q}$ is nontrivial, $S_{q}=\left\{u, y_{i}\right\}$, and $\left(G_{q}, y_{i}, w_{q-1}, u, w_{q}\right)$ is planar. By Lemma (2.7) (with $G_{q}, u, w_{q}, y_{i}, w_{q-1}$ as $G, a, a^{\prime}, b, b^{\prime}$, respectively) there exists a non-separating induced $u$ $w_{q}$ path $Q_{q}$ in $G_{q}$ such that $V\left(Q_{q}\right) \cap\left\{y_{i}, w_{q-1}\right\}=\emptyset$. Moreover, such a path can be found in $O\left(\left|V\left(G_{q}\right)\right|+\left|E\left(G_{q}\right)\right|\right)$ time.

Similarly, if $B_{t}$ is trivial, then let $Q_{t}$ be the path induced by the edge $x_{i} w_{t-1}$. Otherwise (by (I)) $B_{t}$ is nontrivial, $S_{t}=\left\{x_{i}, y_{i}\right\}$, and $\left(G_{t}, y_{i}, w_{t-1}, x_{i}, w_{t}\right)$ is planar. By Lemma (2.7) (with $G_{t}, x_{i}, w_{t-1}, y_{i}, w_{t}$ as $G, a, a^{\prime}, b, b^{\prime}$, respectively) there exists a nonseparating induced $x_{i}-w_{t-1}$ path $Q_{t}$ in $G_{t}$ such that $V\left(Q_{t}\right) \cap\left\{y_{i}, w_{t}\right\}=\emptyset$. Moreover, such a path can be found in $O\left(\left|V\left(G_{t}\right)\right|+\left|E\left(G_{t}\right)\right|\right)$ time.

Let $P^{\prime}:=Q_{q} \cup Z_{q+1} \cup \ldots \cup Z_{t-1} \cup Q_{t} \cup P\left[a, x_{i}\right]$. Then $P^{\prime}$ is a $u$ - $a$ path which is internally disjoint from $V\left(B_{P}\right) \cup V(F)$. Moreover, it is not hard to see that such a path can be found in $O(|V(G)|+|E(G)|)$ time.

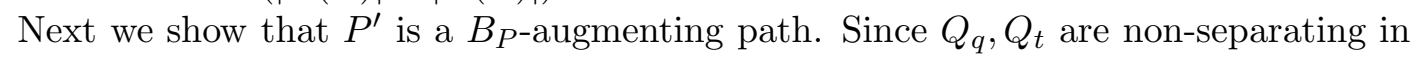
$G_{q}, G_{t}$, respectively, it is not hard to see that $P^{\prime}$ is an induced path in $G-u a$. Because of the path $W$ and since $P\left(a, a^{\prime}\right)$ is non-separating in $G_{F}, P^{\prime}(u, a)$ is non-separating in $G_{F}$. If $a=r$, then $r$ is not a cut vertex of $G_{F}-V\left(P\left(a, a^{\prime}\right)\right)$, and because of the path $W, r$ is not a cut vertex of $G_{F}-V\left(P^{\prime}(u, a)\right)$. Thus, $P^{\prime}$ is a feasible $F$-path. Since $V\left(P^{\prime}\right) \cap V\left(P_{i}-x_{i}\right)=\emptyset, B_{P} \cup W \subseteq B_{P^{\prime}}$. Therefore, $P^{\prime}$ is a $B_{P^{-a u g m e n t i n g ~ p a t h . ~}}$

Subcase 2.3: $x_{i}$ has at most one neighbor in $V\left(B_{l}\right)$ and $x_{i}$ has no neighbor in $V\left(A_{i}\right)-$ $\left(V\left(P\left(x_{i}, y_{i}\right)\right) \cup\left\{b_{i}\right\}\right)$.

In this case, since $A_{i}$ is 2-connected, $b_{i}$ is the only neighbor of $x_{i}$ in $A_{i}$ not contained in $V\left(P\left(x_{i}, y_{i}\right)\right)$. We consider two cases according to whether $x_{i}=a$ or $x_{i} \neq a$.

(A) $x_{i}=a$.

Then by the definition of $x_{i}$ in $(3.9), b_{i} \neq r_{i}$. Since $V\left(A_{i}\right)-\left\{b_{i}, x_{i}, y_{i}\right\}$ has a neighbor in $V(F-r)-\left\{a, a^{\prime}\right\}$ and (III) holds, there exists some $q \in\{1, \ldots, l-1\}$ such that 
$V\left(B_{q}-w_{q-1}\right)$ has a neighbor in $V(F-r)-\left\{a, a^{\prime}\right\}$. Choose $q$ to be minimum with this property.

Therefore, since $b_{i}$ is the only neighbor of $x_{i}$ in $A_{i}$ not contained in $V\left(P_{i}\right)$ and (I) holds, $B_{j}$ is trivial for every $j \in\{1, \ldots, q-1\}$. Using (II), one can show that either $q=1$ or $q=2$. For each $j \in\{1, \ldots, q-1\}$ let $Z_{j}$ be the path induced by the edge $w_{j-1} w_{j}$.

If $B_{q}$ is trivial (in this case $q=1$ ), then, by the choice of $q, w_{q}$ has a neighbor $u$ in $V(F-r)$, and let $Q_{q}:=\left(w_{q-1}, w_{q}, u\right)$. If $B_{q}$ is nontrivial, then by (I) $S_{q}=$ $\left\{u, y_{i}\right\}$ for some $u \in V(F-r)-\left\{a, a^{\prime}\right\}$, and $\left(G_{q}, y_{i}, w_{q-1}, u, w_{q}\right)$ is planar. Note that $u \neq a$ because $x_{i}$ has no neighbor in $V\left(A_{i}\right)-\left(V\left(P_{i}\right) \cup\left\{b_{i}\right\}\right)$. By Lemma (2.7) (with $G_{q}, u, w_{q-1}, y_{i}, w_{q}$ as $G, a, a^{\prime}, b, b^{\prime}$, respectively), there exists a non-separating induced $u$ $w_{q-1}$ path $Q_{q}$ in $G_{q}$ such that $V\left(Q_{q}\right) \cap\left\{y_{i}, w_{q}\right\}=\emptyset$. Moreover, such a path can be found in $O\left(\left|V\left(G_{q}\right)\right|+\left|E\left(G_{q}\right)\right|\right)$ time.

Let $P^{\prime}:=\left(Z_{1} \cup \ldots \cup Z_{q-1} \cup Q_{q} \cup P\left[a, x_{i}\right]\right)+\left\{x_{i}, x_{i} b_{i}\right\}$. Then $P^{\prime}$ is a $u$ - $a$ path which is internally disjoint from $V\left(B_{P}\right) \cup V(F)$. Moreover, it is not hard to see that such a path can be found in $O(|V(G)|+|E(G)|)$ time.

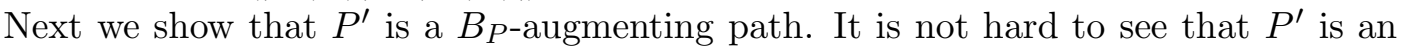
induced path in $G-u a$. Because of the path $W$ and, since $P\left(a, a^{\prime}\right)$ is non-separating in $G_{F}$ and $Q_{q}$ is non-separating in $G_{q}, P^{\prime}(u, a)$ is non-separating in $G_{F}$. If $a=r$, then $r$ is not a cut vertex of $G_{F}-V\left(P\left(a, a^{\prime}\right)\right)$, and because of the path $W, r$ is not a cut vertex of $G_{F}-V\left(P^{\prime}(u, a)\right)$. Thus, $P^{\prime}$ is a feasible $F$-path. Since $B_{P} \cup W \subseteq B_{P^{\prime}}, P^{\prime}$ is a $B_{P}$-augmenting path.

(B) $x_{i} \neq a$.

In this case, it is possible that $b_{i}=r_{i}$. Note that $x_{i}$ has degree at least four in $G$ (because $G$ is 4-connected), $P$ is induced in $G-a a^{\prime}$, and $x_{i}$ has no neighbor in $V\left(A_{i}\right)-$ $\left(V\left(P_{i}\right) \cup\left\{b_{i}\right\}\right)$ (by assumption in this subcase). So $x_{i}$ has a neighbor in $\left(\bigcup_{j=1}^{p} V_{j}\right)-V_{i}$. Let $t \in\{1, \ldots, p\}-\{i\}$ such that $x_{i}$ has a neighbor in $V_{t}$.

Suppose that for some $j \in\{1, \ldots, l-1\}, B_{j}$ is nontrivial. Then by (I) and by our assumption that $x_{i}$ has no neighbor in $V\left(A_{i}\right)-\left(V\left(P\left(x_{i}, y_{i}\right)\right) \cup\left\{b_{i}\right\}\right), S_{j}=\left\{u, y_{i}\right\}$ for some $u \in V(F-r)$, and $\left(G_{j}, y_{i}, w_{j-1}, u, w_{j}\right)$ is planar. Note that $u \neq a^{\prime}$ by the definition of $y_{i}$ in (3.9) and because $u \neq y_{i}$. Also $u \neq a$, because $x_{i} \neq a$. By Lemma (2.6) (with $G_{j}, y_{i}, u, w_{j-1}, w_{j}$ as $G, a, a^{\prime}, b, b^{\prime}$, respectively) there exists a non-separating induced $u-y_{i}$ path $Q$ in $G_{j}$ such that $G_{j}-V(Q)$ has exactly two components $K$ and $K^{\prime}$ with $w_{j-1} \in$ $V(K)$ and $w_{j} \in V\left(K^{\prime}\right)$. Moreover, such a path can be found in $O\left(\left|V\left(G_{j}\right)\right|+\left|E\left(G_{j}\right)\right|\right)$ time (and hence, in $O(|V(G)|+|E(G)|)$ time). Let $P^{\prime}:=Q \cup P\left[y_{i}, a^{\prime}\right]$. Then $P^{\prime}$ is a $u-a^{\prime}$ path in $G$ such that $V\left(P^{\prime}\right) \cap V(F)=\left\{u, a^{\prime}\right\}$. Moreover, it is not hard to see that such a path can be found in $O(|V(G)|+|E(G)|)$ time.

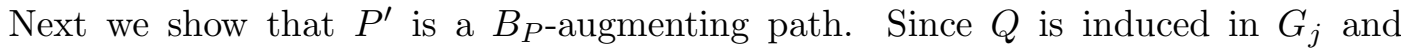
$N_{G}\left(P\left(a, a^{\prime}\right)\right) \cap V(F) \subseteq\left\{a, a^{\prime}\right\} \cup\{r\}$ (by (3.4)), and because $P$ is an induced path in $G-a a^{\prime}$ and $\left.P\left(y_{i}, a^{\prime}\right]\right)$ has no neighbor in $V\left(B_{j}\right)$ (by the definition of $y_{i}$ in $(3.9)$ ), one can 
see that $P^{\prime}$ is an induced path in $G-u a^{\prime}$. Since $G_{j}-V(Q)$ has exactly two components, one containing $w_{j-1}$ and the other containing $w_{j}$, and because $x_{i}$ has a neighbor in $V_{t}$, it is not hard to show that $P^{\prime}$ is non-separating in $G_{F}$. If $r \in\left\{u, a^{\prime}\right\}$, then $r=a^{\prime}$ and $r$ is not a cut vertex of $G_{F}-V\left(P\left(a, a^{\prime}\right)\right)$. In this case, because $x_{i}$ has a neighbor in $V_{t}, r$ is not a cut vertex of $G_{F}-V\left(P^{\prime}\left(u, a^{\prime}\right)\right)$. Thus, $P^{\prime}$ is a feasible $F$-path. Moreover, since $b_{i}$ is adjacent to $x_{i}$ and $x_{i}$ has a neighbor in $V_{t}$, it follows that $P^{\prime}$ is a $B_{P^{-a u g m e n t i n g}}$ path.

Thus, we may assume that $B_{j}$ is trivial for every $j \in\{1, \ldots, l-1\}$. If $l \geq 3$, then $B_{1}$ and $B_{2}$ are trivial, and by (II), $N_{G}\left(w_{1}\right)-\left\{w_{0}, w_{2}\right\}=\left\{u, y_{i}\right\}$ for some $u \in V(F-r)$. Note that $u \notin\left\{a, a^{\prime}\right\}$ because $x_{i} \neq a$ and $y_{i} \neq u$. By an argument similar to the above paragraph, one can show that $P^{\prime}:=\left(u, w_{1}, y_{i}\right)$ is a $B_{P}$-augmenting path.

So we may assume that $l=2$, and $B_{1}$ is trivial. This implies that $V\left(P\left(x_{i}, y_{i}\right)\right) \subseteq$ $V\left(B_{2}\right)$. Hence, $B_{2}$ is nontrivial, so $S_{2}=\left\{x_{i}, y_{i}\right\}$ (by (III)). Since $V\left(A_{i}\right)-\left\{b_{i}, x_{i}, y_{i}\right\}$ has a neighbor in $V(F-r)-\left\{a, a^{\prime}\right\}$ (by assumption in this lemma), and (III) holds, $w_{1}$ is adjacent to some $u \in V(F-r)-\left\{a, a^{\prime}\right\}$. Let $x^{\prime}, y^{\prime}$ denote respectively the vertices in $N_{D}\left(b^{\prime}\right)$ (see $\left.(3.9)\right)$ which are the lowest and the highest in $P$. Since $B_{2}$ is 2-connected, $V\left(B_{2}\right)-\left(V\left(P\left(x_{i}, y_{i}\right)\right) \cup\left\{b^{\prime}\right\}\right)$ has a neighbor in $V\left(P\left(x^{\prime}, y_{i}\right)\right)$. Since $B_{2}-\left(V\left(P\left(x_{i}, y_{i}\right)\right) \cup\right.$ $\left\{b^{\prime}\right\}$ ) is connected (because $A_{i}-V\left(P_{i}\right)$ is connected), there exists a $w_{1}-y_{i}$ path $Q^{\prime}$ in $G_{2}$ such that $x_{i}$ and $b^{\prime}$ are contained in a component $U$ of $G_{2}-V\left(Q^{\prime}\right)$. Moreover, recall that $G_{2}$ is $\left(3,\left\{w_{1}, x_{i}, y_{i}, b^{\prime}\right\}\right)$-connected. Thus, the hypotheses of Lemma (2.1) are satisfied with $G_{2},\left\{w_{1}, x_{i}, y_{i}, b^{\prime}\right\}, w_{1}, y_{i}, U$ as $G, S, a, a^{\prime}, U$, respectively. By (2.1) there exists a non-separating induced $w_{1}-y_{i}$ path $Q$ in $G_{2}$ such that $V(Q) \cap V(U)=\emptyset$ (and hence, $\left.V(Q) \cap\left\{x_{i}, b^{\prime}\right\}=\emptyset\right)$. Moreover, such a path can be found in $O\left(\left|V\left(G_{2}\right)\right|+\left|E\left(G_{2}\right)\right|\right)$ time (and hence, in $O(|V(G)|+|E(G)|)$ time). Let $P^{\prime}:=\left(P\left[y_{i}, a^{\prime}\right] \cup Q\right)+\left\{u, u w_{1}\right\}$. Then $P^{\prime}$ is a $u$ - $a^{\prime}$ path in $G$ such that $V\left(P^{\prime}\right) \cap V(F)=\left\{u, a^{\prime}\right\}$. Moreover, it is not hard to see that such a path can be found in $O(|V(G)|+|E(G)|)$ time.

We conclude the proof by showing that $P^{\prime}$ is a $B_{P}$-augmenting path. Since $Q$ is induced in $G_{2}$ and $N_{G}\left(P\left(a, a^{\prime}\right)\right) \cap V(F) \subseteq\left\{a, a^{\prime}\right\} \cup\{r\}$ (by (3.4)), and because $P$ is an induced path in $G-a a^{\prime}$ and $P\left(y_{i}, a^{\prime}\right]$ ) has no neighbor in $V\left(B_{2}\right)$ (by the definition of $y_{i}$ in (3.9)), one can see that $P^{\prime}$ is an induced path in $G-u a^{\prime}$. Since $G_{2}-V(Q)$ is connected, and because $x_{i}$ has a neighbor in $V_{t}$, it is not hard to see that $P^{\prime}$ is non-separating in $G_{F}$. If $r \in\left\{u, a^{\prime}\right\}$, then $r=a^{\prime}$, and $r$ is not a cut vertex of $G_{F}-V\left(P\left(a, a^{\prime}\right)\right)$. In this case, because $x_{i}$ has a neighbor in $V_{t}, r$ is not a cut vertex of $G_{F}-V\left(P^{\prime}\left(u, a^{\prime}\right)\right)$. Thus, $P^{\prime}$ is a feasible $F$-path. Moreover, since $b_{i}$ is adjacent to $x_{i}$ and $x_{i}$ has a neighbor in $V_{t}$, it follows that $P^{\prime}$ is a $B_{P^{-a u g m e n t i n g ~ p a t h . ~}}$

We are now ready to prove the main result of this section, which implies Theorem (3.1). Consider Algorithm 1.

(3.16) Theorem. Algorithm 1 is correct and runs in $O(|V(G)||E(G)|)$ time. 


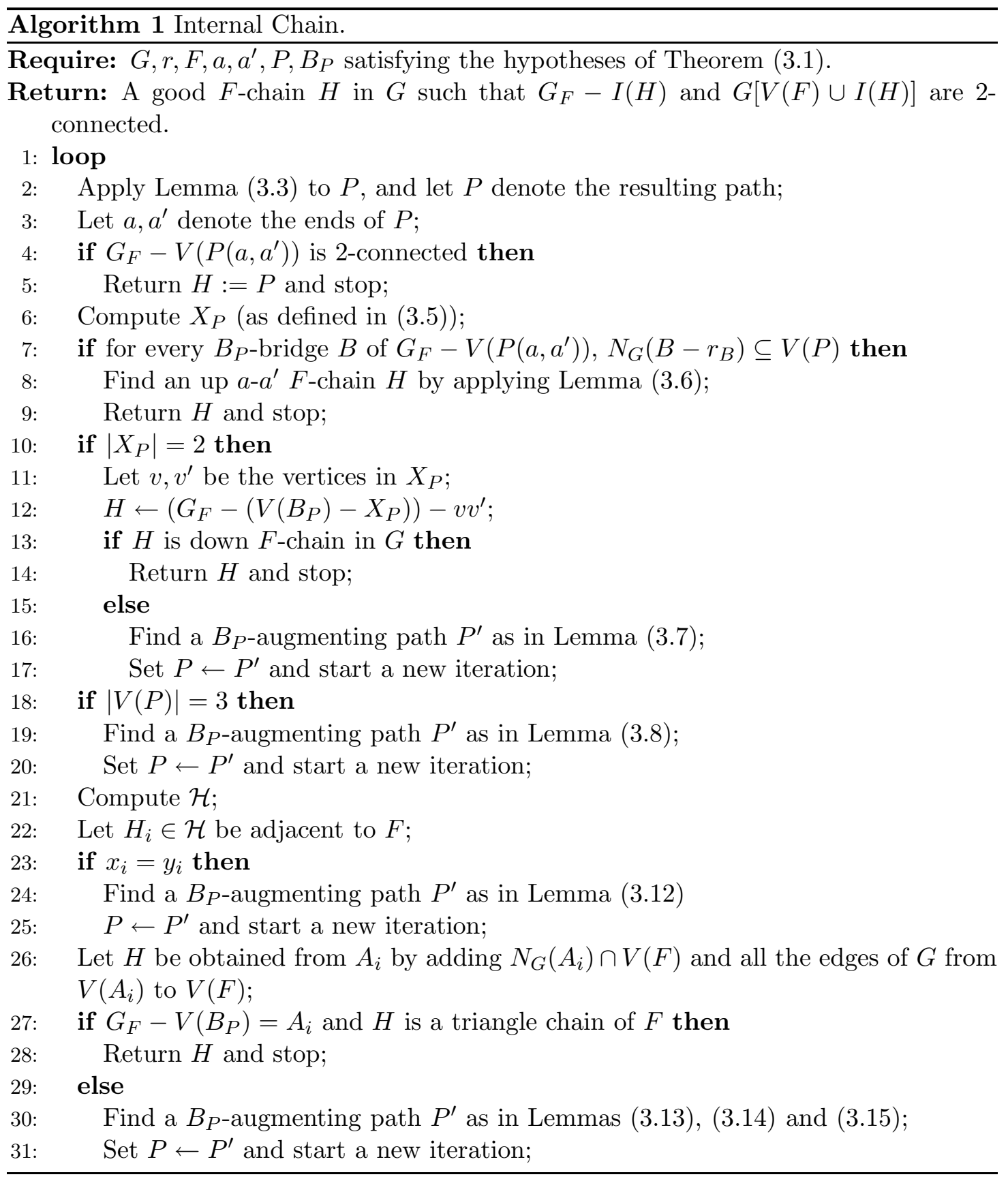


Proof. First, we will prove the correctness of the algorithm.

At the start of each iteration of the main loop, $P$ is a feasible $a-a^{\prime} F$-path and $B_{P}$ is a nontrivial block of $G_{F}:=G-V(F-r)$ containing $r$. As the algorithm progresses $\left|V\left(B_{P}\right)\right|$ increases.

If $G_{F}-V\left(P\left(a, a^{\prime}\right)\right)$ is 2-connected, then the algorithm stops at line 5. Since $P$ is an induced path in $G_{F}-a a^{\prime}, H:=P$ is either an elementary $F$-chain or an up $a-a^{\prime}$ $F$-chain whose blocks are all trivial. Moreover, $G_{F}-I(H)=G_{F}-V\left(P\left(a, a^{\prime}\right)\right)$ and $G[V(F) \cup I(H)]=F \cup P$ are 2-connected.

If for every $B_{P}$-bridge $B$ of $G_{F}-V\left(P\left(a, a^{\prime}\right)\right), N_{G}\left(B-r_{B}\right) \subseteq V(P)$, then by Theorem (3.6) the $a-a^{\prime} F$-chain $H$ in line 8 exists, and $G_{F}-I(H)$ and $G[V(F) \cup I(H)]$ are 2-connected. Thus, if the algorithm stops at line 9 , it returns a correct answer.

If $\left|X_{P}\right|=2$, then by Lemma (3.7) either the subgraph $H$ defined in line 12 is a down $F$-chain, or there exists a $B_{P}$-augmenting path. Thus, if the algorithm stops at line 14 , then $H$ is a down $F$-chain, and $G_{F}-I(H)=B_{P}$ and $G[V(F) \cup I(H)]$ are 2-connected. Otherwise, the algorithm increases $B_{P}$ by executing lines 16-17.

In line 19, if $|V(P)|=3$ (and hence, $\left|X_{P}\right| \geq 3$ ), then by Lemma (3.8) a $B_{P^{-}}$ augmenting path exists, and the algorithm increases $B_{P}$.

Suppose then that $\left|X_{P}\right| \geq 3$ and $|V(P)| \geq 4$. Let $H_{i} \in \mathcal{H}$ be adjacent to $F$ (see Definition (3.9)). If $x_{i}=y_{i}$ then by Lemma (3.12) the $B_{P}$-augmenting path in line 24 exists, and the algorithm increases $B_{P}$. If $x_{i} \neq y_{i}$, then by Lemmas (3.13), (3.14), and (3.15) either the subgraph $H$ defined in line 26 is a triangle chain, or there exists a $B_{P}$-augmenting path. Thus, if the algorithm stops at line 28 , then $H$ is a triangle $F$ chain such that $G_{F}-I(H)=B_{P}$ and $G[V(F) \cup I(H)]$ are 2-connected. Otherwise, the algorithm increases $B_{P}$ by executing lines 30-31.

Since $\left|V\left(B_{P}\right)\right|$ increases at each iteration, the main loop at line 1 eventually stops and a good $F$-chain in $G$ is returned. Hence, Algorithm 1 is correct.

Now we discuss the running time of the algorithm.

The loop in line 1 is executed at most $|V(G)|$ times, since $\left|V\left(B_{P}\right)\right|$ increases at each iteration.

By Lemma (3.3), the step in line 2 can be performed in $O(|V(G)|+|E(G)|)$ time.

The test in line 4 and the steps in line 6 can be executed in $O(|V(G)|+|E(G)|)$ time by standard graph search techniques [18].

The steps in lines 7-9 can be executed in $O(|V(G)|+|E(G)|)$ time by Lemma (3.6).

The steps in lines 10-17 can be executed in $O(|V(G)|+|E(G)|)$ time by Lemma (3.7).

The steps in lines 18-20 can be executed in $O(|V(G)|+|E(G)|)$ time by Lemma (3.8).

The steps in lines 21-22 can be executed in in $O(|V(G)|+|E(G)|)$ time by standard graph search techniques [18].

The steps in lines 23-25 can be executed in $O(|V(G)|+|E(G)|)$ time by Lemma (3.12). 
Finally, the steps in lines 26-31 can be executed in $O(|V(G)|+|E(G)|)$ time by Lemmas (3.13), (3.14), and (3.15).

Therefore, the running time of the Algorithm 1 is $O(|V(G)||E(G)|)$.

\section{Chain decomposition}

In this section, we describe how to construct a non-separating chain decomposition of a 4-connected graph $G$.

The idea is the following. Suppose we have found a partial chain decomposition $H_{1}, H_{2}, \ldots, H_{i-1}$ of $G$, and we want to find the next chain $H_{i}$. Let $F:=G\left[\bigcup_{j=1}^{i-1} I\left(H_{j}\right)\right]$, and assume that $G_{F}:=G-(V(F)-\{r\})$ is 2-connected. If $G_{F}$ is a planar cyclic chain rooted at $r$, then we obtain our desired decomposition by taking $H_{i}:=G_{F}$ and $t:=i$. If $G_{F}$ is not a planar cyclic chain, then we want to use Theorem (3.1). In order to apply it, we need find efficiently vertices $a, a^{\prime} \in V(F)$ and a feasible $a-a^{\prime} F$-path $P$. This will follow from Lemma (4.2) below.

We need the following result, proved in [20] and [2], which was used in [1].

(4.1) Theorem. Let $G$ be a 3-connected graph, let $e \in E(G)$, and let $u \in V(G)$ be non-incident to $e$. Then there exists a non-separating induced cycle in $G$ through $e$ and avoiding $u$. Moreover, such a cycle can be found in $O(|V(G)|+|E(G)|)$ time.

(4.2) Lemma. Let $G$ be a 4-connected graph, let $r \in V(G)$ and let $F$ be a connected subgraph of $G$ such that $r \in V(F),|V(F)| \geq 2$, and $G_{F}:=G-(V(F)-\{r\})$ is 2-connected. Then one of the following holds:

(1) $G_{F}$ is a planar cyclic chain in $G$ rooted at $r$; or

(2) there exists a feasible $a-a^{\prime} F$-path $P$ in $G$, that is,

(i) $V(P) \cap V(F)=\left\{a, a^{\prime}\right\}$ and $P$ is an induced path in $G-a a^{\prime}$,

(ii) $P\left(a, a^{\prime}\right)$ is non-separating in $G_{F}$,

(iii) $r$ is contained in a nontrivial block of $G_{F}-V\left(P\left(a, a^{\prime}\right)\right)$, and

(iv) if $r \in\left\{a, a^{\prime}\right\}$, then $r$ is not a cut vertex of $G_{F}-V\left(P\left(a, a^{\prime}\right)\right)$.

Moreover, one can in $O(|V(G)|+|E(G)|)$ time certify that (1) holds or find a path as in (2).

Proof. First, suppose that $G_{F}$ is 3-connected. Let $G^{\prime}$ be obtained from $G$ by contracting $F-r$ to a single vertex, say $v^{\prime}$. Then $G^{\prime}$ is 4-connected; otherwise, there exists a 3-cut $T$ in $G^{\prime}$. Since $G$ is 4 -connected, $v^{\prime} \in T$. But then $T-\left\{v^{\prime}\right\}$ is a 2-cut in $G_{F}$, which 
is a contradiction. By Theorem (4.1), we can find a non-separating induced cycle $C$ in $G^{\prime}$ through $r v^{\prime}$ in $O(|V(G)|+|E(G)|)$ time. The path $C-r v^{\prime}$ in $G^{\prime}$ corresponds to an induced path $P$ in $G$ from $r$ to some vertex $a^{\prime} \in V(F-r)$. Since $G$ is 4-connected, $r$ has at least two neighbors in $G_{F}-V\left(P\left(r, a^{\prime}\right)\right)$. Moreover, since $C$ is non-separating in $G^{\prime}, r$ is not a cut vertex of $G_{F}-V\left(P\left(r, a^{\prime}\right)\right)$, and $r$ is contained in a nontrivial block of $G_{F}-V\left(P\left(r, a^{\prime}\right)\right)$. Thus, $P, a:=r$, and $a^{\prime}$ satisfy (2).

So we may assume that $G_{F}$ is 2-connected but not 3-connected. Let $\left\{b, b^{\prime}\right\}$ be a 2-cut of $G_{F}$. Let $H_{1}, H_{2}$ be edge-disjoint subgraphs of $G_{F}$ such that $r \in V\left(H_{1}\right), V\left(H_{1}\right) \cap$ $V\left(H_{2}\right)=\left\{b, b^{\prime}\right\}, H_{1} \cup H_{2}=G_{F},\left|V\left(H_{1}\right)\right| \geq 3$, and $\left|V\left(H_{2}\right)\right| \geq 3$. Choose $H_{1}, H_{2}$ such that $H_{2}$ is minimal. Note that $b, b^{\prime}, H_{1}, H_{2}$ can be found in $O(|V(G)|+|E(G)|)$ time using the algorithm in [6] for finding the 3-connected components of $G_{F}$. Let $S:=N_{G}\left(H_{2}-\left\{b, b^{\prime}\right\}\right)-\left\{b, b^{\prime}\right\}$, and let $G^{\prime}$ be obtained from $H_{2}$ by adding $S$ and the edges of $G$ from $S$ to $V\left(H_{2}\right)-\left\{b, b^{\prime}\right\}$. Note that $S \subset V(F),|S| \geq 2$, and $r \notin S$ because $\left\{b, b^{\prime}\right\}$ is a 2 -cut of $G_{F}$ and $r \notin V\left(H_{2}\right)-\left\{b, b^{\prime}\right\}$. Moreover, $G^{\prime}$ is $\left(4, S \cup\left\{b, b^{\prime}\right\}\right)$-connected.

Suppose that $\left|V\left(H_{2}\right)\right| \geq 4$. Then by minimality of $H_{2}, H_{2}$ is 2-connected and $G^{\prime}, b, b^{\prime}, S$ satisfy (i)-(v) in Lemma (2.3) (with $G^{\prime}$ as $G$ ). Therefore, we can in $O\left(\left|V\left(G^{\prime}\right)\right|+\left|E\left(G^{\prime}\right)\right|\right)$ time either

(I) find $a, a^{\prime} \in S$ and an induced $a$ - $a^{\prime}$ path $P^{\prime}$ in $G^{\prime}$ such that $V\left(P^{\prime}\right) \cap\left\{b, b^{\prime}\right\}=\emptyset$, $V\left(P^{\prime}\right) \cap S=\left\{a, a^{\prime}\right\}$, and $G-\left(V\left(P^{\prime}\right) \cup S\right)$ is connected, or

(II) certify that $|S|=2$, and the vertices in $S$ can be labeled as $a, a^{\prime}$ such that $\left(G^{\prime}, a, b, a^{\prime}, b^{\prime}\right)$ is planar.

If (I) occurs, then $r$ is contained in a nontrivial block of $G-V\left(P^{\prime}\right)$, since there exists a $b$ - $b^{\prime}$ path in $H_{2}-V\left(P\left(a, a^{\prime}\right)\right)$. Since $r \notin S$, we have $r \notin\left\{a, a^{\prime}\right\}$. Hence, $P:=P^{\prime}$ is a path that satisfies (2).

So we may assume that one of the following holds: $\left|V\left(H_{2}\right)\right| \geq 4$ and (II) occurs, or $\left|V\left(H_{2}\right)\right|=3$.

We claim that one can find in $O\left(\left|V\left(G^{\prime}\right)\right|+\left|E\left(G^{\prime}\right)\right|\right)$ time a path $P$ in $G^{\prime}$ with ends $a, a^{\prime}$ in $S$ such that $G^{\prime}-(V(P) \cup S)$ has exactly two components $K, K^{\prime}$ with $b \in V(K)$ and $b^{\prime} \in V\left(K^{\prime}\right)$. If $\left|V\left(H_{2}\right)\right| \geq 4$ and (II) occurs, then this follows from Lemma (2.6). If $\left|V\left(H_{2}\right)\right|=3$ then let $v$ be the only vertex in $V\left(H_{2}\right)-V\left(H_{1}\right)$. Then $v$ has degree two in $G_{F}$, and since $G$ is 4 -connected, $v$ has at least two neighbors in $V(F)$, say $a, a^{\prime}$. Then $P:=\left(a, v, a^{\prime}\right)$ is the required path.

Therefore, $G_{F}-V\left(P\left(a, a^{\prime}\right)\right)$ is connected. If $r$ is contained in a nontrivial block of $H_{1}$, then $r$ is contained in a nontrivial block of $G_{F}-V\left(P\left(a, a^{\prime}\right)\right)$, and since $r \notin S, r \notin\left\{a, a^{\prime}\right\}$. In this case, $P$ satisfies (2).

So assume that $r$ is contained only in trivial blocks of $H_{1}$.

Since $G_{F}$ is 2-connected, $H_{1}$ is a $b$ - $b^{\prime}$ chain. Moreover, either $r$ is a cut vertex of $H_{1}$, or $r \in\left\{b, b^{\prime}\right\}$. In both cases, $G_{F}$ is a cyclic chain rooted at $r$. Let $G_{F}:=v_{0} B_{1} v_{1} \ldots v_{k-1} B_{k} v_{k}$ 
for some integer $k \geq 2$ (where $v_{0}=v_{k}=r$ ). Note that either $H_{2}=B_{j}$ for some $1 \leq j \leq k$ (when $\left|V\left(H_{2}\right)\right| \geq 4$ ), or $H_{2}=B_{j} \cup B_{j+1}$ for some $1 \leq j \leq k-1$ where $B_{j}, B_{j+1}$ are trivial (when $\left|V\left(H_{2}\right)\right|=3$ ).

If all the $B_{i}$ 's are trivial, then $G_{F}$ is a planar cyclic chain, and (2) holds. So assume that not all $B_{i}$ 's are trivial. For each 2-connected $B_{i}$, let $S_{i}:=N_{G}\left(B_{i}-\right.$ $\left.\left\{v_{i-1}, v_{i}\right\}\right)-\left\{v_{i-1}, v_{i}\right\}$, and let $G_{i}$ be obtained from $B_{i}$ by adding $S_{i}$ and the edges of $G$ from $S_{i}$ to $V\left(B_{i}\right)$. Then $S_{i} \subseteq V(F-r)$, because $\left\{v_{i-1}, v_{i}\right\}$ is a 2-cut of $G_{F}$, and $r \notin V\left(B_{i}\right)-\left\{v_{i-1}, v_{i}\right\}$. Note that $G_{i}, S_{i}, v_{i-1}, v_{i}$ (as $G, S, b, b^{\prime}$, respectively) satisfy (i)-(v) of Lemma (2.3) because $G_{i}-S_{i}=B_{i}$ is 2-connected and $G_{i}$ is $\left(4, S_{i} \cup\left\{v_{i-1}, v_{i}\right\}\right.$ )connected. Thus, one can in $O\left(\left|V\left(G_{i}\right)\right|+\left|E\left(G_{i}\right)\right|\right)$ time either (a) find $a_{i}, a_{i}^{\prime} \in S_{i}$ and an induced $a_{i}-a_{i}^{\prime}$ path $P_{i}$ in $G$ such that $V\left(P_{i}\right) \cap\left\{v_{i-1}, v_{i}\right\}=\emptyset, V\left(P_{i}\right) \cap S_{i}=\left\{a_{i}, a_{i}^{\prime}\right\}$, and $G_{i}-\left(V\left(P_{i}\right) \cup S_{i}\right)=B_{i}-V\left(P_{i}\left(a_{i}, a_{i}^{\prime}\right)\right)$ is connected, or (b) certify that $\left|S_{i}\right|=2$, and the vertices in $S_{i}$ can be labeled as $a_{i}, a_{i}^{\prime}$ such that $\left(G_{i}, v_{i-1}, a_{i}, v_{i}, a_{i}^{\prime}\right)$ is planar. Since $G$ is 4-connected, if (b) occurs then $B_{i}-\left\{v_{i-1}, v_{i}\right\}=G_{i}-\left(S_{i} \cup\left\{v_{i-1}, v_{i}\right\}\right)$ is connected.

If $G_{F}$ is not a planar cyclic chain rooted at $r$, then (a) must hold for some 2-connected $B_{i}$, and hence, $P:=P_{i}$ satisfies (2) (because $r \notin S_{i}$ ). Otherwise, (1) holds.

It is not hard to see that all the steps described above can be executed in $O(|V(G)|+$ $|E(G)|)$ time.

Thus, combining Lemma (4.2) and Theorem (3.1) we obtain the following.

(4.3) Theorem. Let $G$ be a 4-connected graph, let $F$ be a subgraph of $G$, and let $r \in V(F)$ such that $G_{F}:=G-(V(F)-\{r\})$ is 2-connected. Then one of the following holds:

(1) there exists a good $F$-chain $H$ in $G$ such that $G_{F}-I(H)$ and $G[V(F) \cup I(H)]$ are 2-connected, or

(2) $G_{F}$ is a planar cyclic chain rooted at $r$.

Moreover, one can in $O(|V(G)|+|E(G)|)$ time find a good F-chain as in (1) or certify that (2) holds.

We are now ready to prove the main result in this paper.

Proof of Theorem (1.5). A non-separating chain decomposition of $G$ starting at $r a$ can be found as follows. The first chain $H_{1}$ can be found in $O(|V(G)||E(G)|)$ time by Theorem (1.6). The internal chains can be found iteratively as follows. Suppose we have found a partial chain decomposition $H_{1}, \ldots, H_{i-1}(i \geq 2)$ of $G$ and we want to find $H_{i}$. Let $F:=G\left[\bigcup_{j=1}^{i-1} I\left(H_{j}\right)\right]$. Apply Theorem (4.3) to $G, F$ and $r$. Then one of the following holds: 
(1) there exists a good $F$-chain $H$ in $G$ such that $G_{F}-I(H)$ and $G[V(F) \cup I(H)]$ are 2-connected, or

(2) $G_{F}$ is a planar cyclic chain rooted at $r$.

Moreover, one can in $O(|V(G)|+|E(G)|)$ time find a planar chain as in (1) or certify that (2) holds. If (1) holds, then let $H_{i}:=H$ and set $i \leftarrow i+1$. If (2) holds, then $H_{1}, \ldots, H_{i}:=G_{F}$ is the desired chain decomposition.

Since the number of chains is at most $|V(G)|$, the above algorithm has time complexity $O\left(|V(G)|^{2}|E(G)|\right)$.

\section{References}

[1] S. Curran, O. Lee and X. Yu, Non-separating planar chains in 4-connected graphs. Submitted.

[2] J. Cheriyan and S. N. Maheshwari, Finding nonseparating induced cycles and independent spanning trees in 3-connected graphs, J. Algorithms 9 (1988), 507537.

[3] N. Chiba and T. Nishizeki, The hamiltonian cycle problem is linear-time solvable for 4-connected planar graphs, J. Algorithms 10 (1989), 187-211.

[4] S. Curran and X. Yu, Non-separating cycles in 4-connected graphs. To appear.

[5] D. Dolev, J. Y. Halpern, B. Simons and R. Strong, A new look at fault tolerant network routing, Proc. 16th Annual ACM Symp. on Theory of Comput. (1984), $526-535$.

[6] J. E. Hopcroft and R. E. Tarjan, Dividing a graph into three connected components, SIAM J. Comput. 2 (1973), 135-158.

[7] J. E. Hopcroft and R. E. Tarjan, Efficient planarity testing, J. Assoc. Comp. Mach. 21 (1974), 549-568.

[8] W. L. Hsu and W. K. Shih, A new planarity test, Theoretical Comp. Sci. 223 (1999), 179-191.

[9] A. Huck, Independent trees in graphs, Graphs and Combinatorics 10 (1994), $29-45$.

[10] T. Ibaraki and H. Nagamochi, A linear-time algorithm for finding a sparse $k$ connected spanning subgraph of a $k$-connected graph, Algorithmica 7 (1992), $583-596$. 
[11] A. Itai and M. Rodeh, The multi-tree approach to reliability in distributed networks, Proc. 25th Annual IEEE Symp. Found. Comp. Sci. (1984), 137-147.

[12] A. Itai and A. Zehavi, Three tree-paths, J. Graph Theory 13 (1989), 175-188.

[13] L. Lovász, Problems in Recent Advances in Graph Theory, (ed. M. Fiedler), Academia, Prague, 1975.

[14] K. Miura, S. Nakano, T. Nishizeki and D. Takahashi, A linear-time algorithm to find four independent spanning trees in four connected planar graphs, Int. J. Found. Comp. Sci. 10 (1999), 195-210.

[15] Y. Perl and Y. Shiloach, Finding two disjoint paths between pairs of vertices in a graph, J. Assoc. Comp. Mach. 25 (1978), 1-9.

[16] P. D. Seymour, Disjoint paths in planar graphs, Discrete Math. 29 (1980), 293309.

[17] Y. Shiloach, A polynomial solution to the undirected two paths problem, $J$. Assoc. Comp. Mach. 27 (1980), 445-456.

[18] R. E. Tarjan, "Data Structures and Network Algorithms", SIAM Monograph, SIAM, Philadelphia, PA, 1983.

[19] C. Thomassen, A theorem on paths in planar graphs, J. Graph Theory 7 (1983), $169-176$.

[20] W. T. Tutte, How to draw a graph, Proc. London Math Soc. 13 (1963), 743-767.

[21] M. Watkins, On the existence of certain disjoint arcs in graphs, Duke Math. J. 35 (1968), 321-346. 